Publié dans: Stress and strain in epitaxy: theoretical concepts, measurements and applications, Ed M Hanbücken, J.P. Deville, Elsevier 2001, p3-61

\title{
Some elastic effects in crystal growth
}

\author{
P. Müller, R. Kern
}

Centre de Recherche sur les Mécanismes de la Croissance Cristalline*

Campus de Luminy, case 913, F-13288 Marseille Cedex 9, France

\begin{abstract}
These lectures deal with some elastic effects in crystal growth. We recall some basics results about the elastic description of a bulk solid and its surface, then we emphasize on surface stress and surface strain quantities and on the description of surface defects in terms of point forces. Then we focus on the morphological stability of a stressed surface and epitaxially strained crystal as well. We will show how surface stress modifies wetting conditions and how bulk stress modifies the equilibrium state. For 2D growth (perfect wetting) bulk strain modifies the chemical potential of each layer and due to finite size wetting we introduce, it results a number of equilibrium layers for each imposed undersaturation.

For 3D growth (no perfect wetting) the epitaxial stress acts against wetting and leads to a global thickening of the equilibrium shape. We also show how elastic relaxation is a prerequisite for the simultaneous existence of 2D layers and 3D crystals (Stranski Krastanov or SK growth). In the three cases of 2D, 3D or SK mixed mode, beyond some critical size, plastic relaxation may occur. In a last part we consider elastic effects on growth mechanisms. We show that, except for Stranski Krastanov growth, the activation barrier for nucleation is not significantly influenced by strain. In contrast strain plays a role on the detachment rate of atoms (strain lowers the barrier to detachment of atoms from laterally large islands in respect to laterally small islands) and then on kinetics. Then we focus on strain effects on step flow growth and show how step-step and/or adatom-step elastic interactions may give birth to a supplementary net force on each adatom. This force modifies the net current of adatoms and thus leads to some new growth instabilities. The surface diffusion coefficient itself may also be modified by strain but without noticeable modification of growth mechanisms. At last we mention some collective effects.
\end{abstract}

*Associé aux Universités Aix-Marseille II et III. 


\section{INTRODUCTION}

Since Royer [1] the regular oriented over-growth of a crystalline material A onto a single crystal surface B is called epitaxy. Two lattice planes of A and B and at least two lattice rows come in contact and in case of coherent epitaxy accommodate their two dimensional (2D) misfit. By this means the couple A/B stores a certain amount of elastic energy. The so-stored elastic energy has been recognised so far as a source of mechanical problems such as cracking, blistering, peeling... Then for many years the main problem of crystal growers was to avoid strain by choosing very low-mismatched systems. Nevertheless it has also been recognised that stress can modify some crystal properties. This is the case of the functional performance of devices such as the possibility of band-gap engineering involving strained structures [2] or the correlation between mechanical stress and magnetic anisotropy in ultra thin films [3]. These technological considerations have stimulated crystal growers to consider also crystal growth properties induced by stress. Nevertheless the problem of formation of a strained crystal on a single crystal is complex. The difficulties basically have three origins.

The first difficulty arises from the fact that since the equilibrium shape of a crystal essentially depends upon surface energy considerations [4,5], a good description of the thermodynamic state of a strained crystal needs to accurately define the role of stress and strain on specific surface energies. This can be done by properly defining surface stress and strain quantities as partially done by Gibbs [6], Shuttleworth [7], Herring [5] and others $[8,9,10]$.

The second difficulty arises from the fact that most strains are anisotropic and inhomogeneous. Indeed on one hand because of the Poisson effect the in-plane strain due to misfit accommodation is accompanied by a vertical opposite strain. Furthermore on the other hand, islands or nuclei can relax by their edges. Obviously this elastic relaxation depends on the shape of the island and therefore cannot be homogeneous! Thus a good description of the bulk elastic energy needs to calculate accurately elastic relaxation.

The third difficulty arises from the fact that, even weak, the elastic effects dominate at long range. Thus elasticity may also affect long-range behaviour usually driven by surface diffusion considerations. In other words not only the energetics of crystal growth may be altered by elasticity but kinetics behaviour may also be altered.

Our purpose in these lectures is to describe some elastic effects on crystal growth. For the sake of simplicity we will only consider pure cubic materials A over B and furthermore do not consider alloy composition, especially changes induced by strain (except briefly in section 3.3.4.). Furthermore as in most of the analytical formulations we will use macroscopic and linear elasticity. For too high misfits $(>1 \%)$ linearity may fail, for studying the first stages of growth as nuclei the macroscopic treatment may be questionable too. The main advantage of linear elasticity is the possible analytic form of the results (even when complex) that gives the basic tendencies. Obviously in a specific treatment theses results have to be compared with atomistic (if better) calculations.

Last but not least, though kinetics may more or less slow down the realization of the final state, thermodynamics remains the primer way to tell what is possible so that in the framework of these lectures we first focus on thermodynamic properties then on kinetics. The lectures are divided into three parts corresponding approximately to the three abovementioned difficulties.

In the first part (section 1.) we will recall some basic results of the classical elasticity theory. Since considering crystal growth and thus surface phenomena, we will focus on the accurate 
description of elastic properties of surfaces. For this purpose we will introduce surface stress and surface strain as surface excess quantities. Furthermore since crystal growth often starts on surface defects (such as steps) we will also describe elastic fields induced by such surface defects (adatoms, steps, domains...)

In the second part (section 2.) we will focus on elasticity effects on the macroscopic thermodynamic state. For this purpose we will revisit Bauer's thermodynamic analysis of epitaxial growth $[11,12]$ by taking into account elastic energy of bulk and surface as well. More precisely we will consider elastic effects on two dimensional (2D), three dimensional (3D) and mixed 3D/2D (or Stranski-Krastanov) growth modes. We will see that, even weak, elasticity may play a major role on the equilibrium properties such as the number of equilibrium layers of $2 \mathrm{D}$ film or the equilibrium aspect ratio of $3 \mathrm{D}$ crystals. More exciting is the fact that, if in absence of elastic relaxation there is no place for the Stranski-Krastanov growth mode in equilibrium conditions (except in case of some exotic structure change or for kinetics reasons) elasticity considerations open a place for equilibrium Stranski-Krastanov transition.

The third part (section 3.) concerns elasticity effects on microscopic or elementary growth mechanisms. For this purpose we will see how elastic interactions may influence nucleation and step flow. In the first case we only have to consider the role of the elastic interactions on the nucleation activation energy. In fact it will be very weak. In the second case we will reconsider usual kinetics formulation of step flow with as a new ingredient an elastic contribution to the net current of adatoms due to step-step or adatom-step elastic interactions. The main effect of elasticity is then to favour the appearance of new kind of surface instabilities.

At last in a short conclusion we will mention some elastic effects we do not take up in detail in these lectures.

\section{ELASTIC DESCRIPTION OF A SOLID AND ITS SURFACE}

\subsection{Elastic description of bulk phases}

When a bulk material is stressed (resp. strained) it becomes strained (resp. stressed). Stress and strain are connected by the elastic constants of the material. Many textbooks deal with elastic properties of solids, fundamental aspects are given in $[13,14]$ whereas [15] essentially focus on anisotropic properties.

In this first section we only recall some fundamental concepts.

\subsubsection{Bulk stress tensor}

Let us consider an elementary parallelepiped (volume $d V=d x_{i} d x_{j} d x_{k}$ ) centred on a point $x_{i}$ in a stressed solid (see figure 1a). Each of its faces ( $a r e a d x_{i} d x_{j}$ ) normal to the $x_{k}(i, j, k=1,2,3)$ direction is submitted to a force per unit area (a pressure when negative) whose $\mathrm{i}^{\text {th }}$ component reads $\sigma_{i k}$ in the homogeneous case or $\sigma_{i k}+1 / 2\left(\partial \sigma_{i k} / \partial x_{k}\right) d x_{k}$ up to the first order in the inhomogeneous case (independent of $\mathrm{x}_{\mathrm{i}}$ ). The bulk stress thus is defined by a third order tensor of rank two $[\sigma]$. The three $\sigma_{i i}$ components describe normal stress whereas $\sigma_{i k}$ components with $i \neq k$ define shearing stresses. The components $\sigma_{i j}$ of $[\sigma]$ are not invariant under axis rotation (only the trace of the tensor whose mean value equals the mean negative 
pressure is invariant). As for all symmetric second rank tensors, the components transform as $\sigma_{m n}^{\prime}=a_{m i} a_{n k} \sigma_{i k}$ where $a_{i j}$ are the components of the matrix of axis transformation [15].

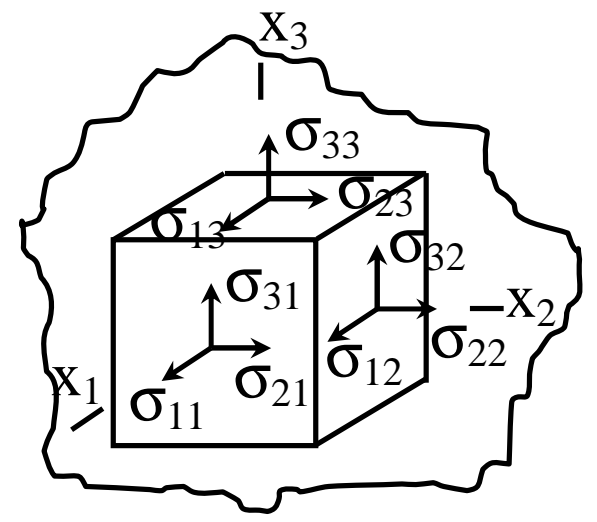

Figure 1a: Action of the components $\sigma_{i j}$ of the bulk stress tensor applied on the three front faces of the elementary cube. Each face normal to $x_{j}$ bears a triplet $\sigma_{1 j}, \sigma_{2 j}, \sigma_{3 j}$, the first index $i=1,2,3$ giving the direction $x_{i}$ where the stress acts. When $i=j$ they are normal stresses, when $i \neq j$ they act in the face. On the back faces of that cube are acting identical stresses of opposite signs or slightly different ones for inhomogeneous stresses.

When the elementary parallelepiped is in mechanical equilibrium that means when no resultant force or torque displaces or rotates it, the bulk stress tensor fulfils the following conditions [15] (see foot note)

$\partial \sigma_{i k} / \partial x_{k}=f_{i}$ and $\sigma_{i k}=\sigma_{k i}$

where $f_{i}$ is the $\mathrm{i}^{\text {th }}$ component of the bulk density of forces. Bulk density of forces generally comes from gravity and can often be neglected when considering nano-crystals. Owing to its diagonal property (1), the bulk stress tensor $[\sigma]$ can also be written as a 6 dimensional vector with $\quad \sigma_{i} \equiv \sigma_{i i}, \quad \sigma_{4} \equiv \sigma_{23} ; \quad \sigma_{5} \equiv \sigma_{13}$ and $\sigma_{6} \equiv \sigma_{12}$ (Voigt notation). However $\sigma_{m}$ components transform differently than $\sigma_{i j}$ components under axis transformation [16].

\subsubsection{Bulk strain tensor}

The symmetric bulk strain tensor components are defined by $\varepsilon_{i k}=\frac{1}{2}\left(\frac{\partial u_{i}}{\partial x_{k}}+\frac{\partial u_{k}}{\partial x_{i}}\right)$ where $\mathrm{u}_{\mathrm{i}}$ are the components of the displacement field $[13,15]$. The artificial symmetrisation of the strain tensor avoids considering a simple rotation as a deformation [15]. The $\varepsilon_{i i}$ components describe the relative elongation of an infinitesimal length parallel to axis $\mathrm{x}_{\mathrm{i}}$, whereas $\pi / 2-2 \varepsilon_{i k}$ with $\mathrm{i} \neq \mathrm{k}$ is the deformation angle measured between two straight lines initially parallel to axis $\mathrm{x}_{\mathrm{i}}$ and $\mathrm{x}_{\mathrm{k}}$ respectively. As for $\sigma_{i i}$, the trace $\varepsilon_{i i}$ is rotation invariant as it should be obviously since it represents the bulk dilatation.

As for the bulk stress tensor one can define a 6 dimensional strain vector as $\varepsilon_{i} \equiv \varepsilon_{i i}, \quad \varepsilon_{4} / 2 \equiv \varepsilon_{23}, \quad \varepsilon_{5} / 2 \equiv \varepsilon_{31}$ and $\varepsilon_{6} / 2 \equiv \varepsilon_{12}$ where the factor 2 is introduced for further simplifications. Let us note that $\varepsilon_{i j}\left(\operatorname{resp} \varepsilon_{m}\right.$ ) components transform as $\sigma_{i j}\left(\operatorname{resp} \sigma_{m}\right)$ components $[15,16]$.

\footnotetext{
${ }^{\dagger}$ We use Einstein notation, thus summation has to be performed on repeated indices.
} 


\subsubsection{Hooke's law}

In the framework of linear elasticity, relationships between stress and strain can be written up to the first order as

$$
\left\{\begin{array}{l}
\sigma_{i k}=C_{i k m n} \varepsilon_{m n} \\
\varepsilon_{i k}=S_{i k m n} \sigma_{m n}
\end{array}\right.
$$

where $\mathrm{C}_{\mathrm{ikmn}}$ and $\mathrm{S}_{\mathrm{ikmn}}$ are called stiffness and compliance coefficients respectively. These coefficients describe the elastic properties of the material. Since stiffness [C] and compliances $[\mathrm{S}]$ are fourth rank tensors, they contain in 3D, 81 components which transform under an axis transformation as $C^{\prime}{ }_{i k m n}=a_{i o} a_{k p} a_{m q} a_{n r} C_{o p q r}$. In fact owing to stress and strain tensor intrinsicsymmetry and energy invariance as well, these tensors only contain 21 independent components. Furthermore crystalline symmetries (extrinsic) reduce the number of independent components from 21 for triclinic crystals to 3 for cubic crystals [15]. Isotropic material (such as glass) are simply described by two elastic constants.

Obviously, using the vectorial (or Voigt) notation of stress and strain tensors, Hooke's law (2) can also be written

$$
\left\{\begin{array}{l}
\sigma_{i}=C_{i k} \varepsilon_{k} \\
\varepsilon_{i}=S_{i k} \sigma_{k}
\end{array}\right.
$$

with i,k=1,2,3,4,5,6. $C_{i k}$ and $S_{i k}$ thus are $6 \times 6$ matrices inverse each other. They are not tensors. Relationships between tensorial and matrix components are $C_{i j k l}=C_{m n}$ with ii=m whatever $\mathrm{i} \neq \mathrm{j}, \mathrm{m}=4$ for $\mathrm{ij}=23, \mathrm{~m}=5$ for $\mathrm{ij}=13$ and $\mathrm{m}=6$ for $\mathrm{ij}=12$. In contrast $S_{i j k l}=S_{m n}$ for $\mathrm{m}$ and $\mathrm{n}$ $=1,2,3 ; 2 S_{i j k l}=S_{m n}$ for $\mathrm{m}$ or $\mathrm{n}=4,5,6 ; 4 S_{i j k l}=S_{m n}$ for $\mathrm{m}$ and $\mathrm{n}=4,5,6$ with as in section 2.1 . $\mathrm{ii}=\mathrm{m}$ whatever $\mathrm{i}, \mathrm{m}=4$ for $\mathrm{ij}=23, \mathrm{~m}=5$ for $\mathrm{ij}=13$ and $\mathrm{m}=6$ for $\mathrm{ij}=12^{\ddagger}$. Elastic constants values are generally given in this Voigt notation for some particular crystallographic orientation $[17,18]$. For other orientations the elastic constants have to be recalculated by the very lengthy transformation of the components of the fourth rank tensors [C] or [S]. Once the transformation has been performed the elastic tensors can be again written in Voigt's notation. Some usual transformations can be found in $[19,20]$, but for a very efficient and general matrix method see Angot [16].

\subsubsection{Bulk elastic energy}

The elastic energy can be defined as the work of the forces per unit area $\left(\sigma_{i k}\right)$ against the bulk deformation $\left(\varepsilon_{i k}\right)$ and thus reads for a material of volume $\mathrm{V}$ [13]

$$
W_{e l}^{B u l k}=\frac{1}{2} \int \sigma_{i k} \varepsilon_{i k} d V
$$

\footnotetext{
\# Let us warn that some authors use different definitions.
} 
For homogeneous stress and strain one thus can obtain with (2) or (3) the energy density (4) under the following equivalent forms

$$
w_{e l .}=\frac{d W_{e l}^{B u l k}}{d V}=\frac{1}{2} C_{i k m n} \varepsilon_{i k} \varepsilon_{m n}=\frac{1}{2} S_{i k m n} \sigma_{i k} \sigma_{m n}=\frac{1}{2} C_{i k} \varepsilon_{i} \varepsilon_{k}=\frac{1}{2} S_{i k} \sigma_{i} \sigma_{k}
$$

In most of the practical cases elastic energy may be roughly estimated by assuming the material to be isotropic and thus only described by two elastic constants $C_{11}\left(S_{11}\right)$ and $C_{12}$ $\left(\mathrm{S}_{12}\right)$ in matrix notation or more currently the Young modulus $E_{i s}^{3 D}$ and Poisson ratio $v_{i s}^{3 D}$ defined by [15]

$$
E_{i s}^{3 D}=\frac{\left(C_{11}-C_{12}\right)\left(C_{11}+2 C_{12}\right)}{C_{11}+C_{12}}=\frac{1}{S_{11}} \quad \text { and } \quad v_{\mathrm{is}}^{3 D}=\frac{C_{12}}{C_{11}+C_{12}}=-\frac{S_{12}}{S_{11}}
$$

For an isotropic material the elastic energy density (5) when isotropically strained $\left(\varepsilon_{i i}=\varepsilon\right.$ ) thus reads after development of (5) with (6)

$$
w_{e l .}=\frac{E_{i s}^{3 D}}{\left(1-v_{i s}^{3 D}\right)} \varepsilon^{2}
$$

For anisotropic crystals, relation (5) and corresponding elastic constants have to be used.

For biaxially strained films things can nevertheless be simplified by introducing two dimensional Young modulus and Poisson ratio. For instance let us consider a (001) biaxially strained layer $\left(\varepsilon_{11}=\varepsilon_{22}=m_{o}\right.$ ) of a cubic crystal. Thus from appendix A there is:

$w_{e l}^{(001)}=E_{(001)}^{2 D} m_{0}^{2}$

with $E_{(001)}^{2 D}=C_{11}+C_{12}-2 \frac{C_{12}^{2}}{C_{11}} \quad$ and $\quad v_{(001)}^{2 \mathrm{D}}=-2 \frac{C_{12}}{C_{11}}$

where $E_{100}^{2 D}=C_{11}+C_{12}-2 C_{12}^{2} / C_{11}$ is the two dimensional Young modulus in the (100) face plane (see appendix A). Notice that in all the cases the elastic energy density is quadratic in respect to strain. For other crystallographic orientations elastic constants have to be transformed under axis rotation. However the elastic energy density of epitaxially strained layers can always be written (in absence of relaxation) under the form

$$
w_{e l}=Y m_{0}^{2}
$$

where $Y$ is a combination of elastic constants $C_{i j}$ or $S_{i j}$. Usually $Y \approx 10^{12} \mathrm{erg} \cdot \mathrm{cm}^{-3}=10^{2} \mathrm{GJm}^{-3}$ the elastic energy density is of the order of the chemical bonding $\left(2 \mathrm{eV} / \mathrm{at} . \approx 0.25 \mathrm{GJm}^{-3}\right)$ for strain $\mathrm{m}_{\mathrm{o}}$ of roughly $5 \%$. Obviously such important energy density cannot be neglected when formulating thermodynamic description of crystal growth, as we will see in section 3 .

\subsection{Elastic description of ideal planar interfaces}

The elastic properties of surfaces can be described by surface stress and strain as excess quantities as first described by Gibbs [6], Shuttleworth [7] and Herring [5] (for surface stress) 
then Andreev and Kosevitch [8] and Nozières and Wolf $[9,10]$ who furthermore introduced surface strain. In the following we will follow Nozières approach.

\subsubsection{Surface stress}

Let us consider an infinite coherent planar interface (whose normal is the axis $x_{3}$ ) in between two bulk phases I and II both characterised by their own homogeneous stress tensor $\sigma_{i k}^{I}$ and $\sigma_{i k}^{I I}$. Since in the infinite interface the stresses cannot depend on in-plane $\mathrm{x}_{1}$ and $\mathrm{x}_{2}$ coordinates, the mechanical equilibrium condition (1), written in absence of bulk forces $f_{i}$ gives at the interface $\left(\mathrm{x}_{3}=0\right) ; \partial \sigma_{i 3} / \partial x_{3}=0$.

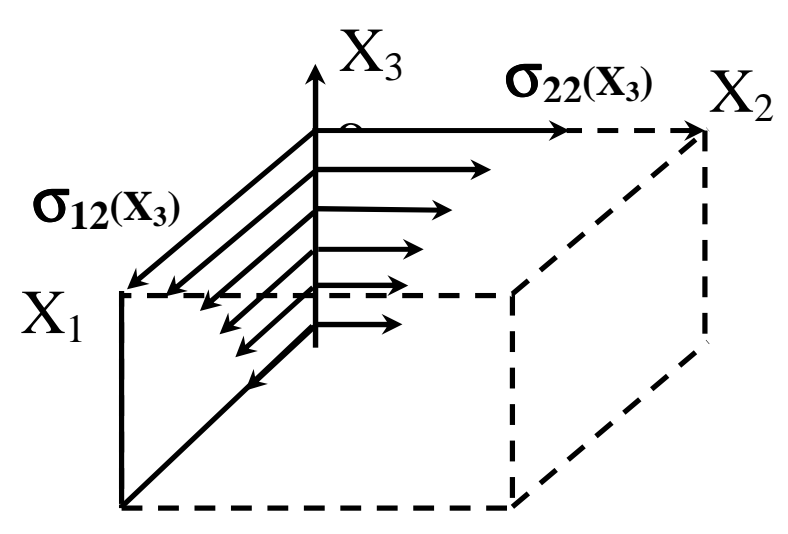

Figure 1b: Surface stress as an excess of bulk stress second rank tensor. Schematically for the surface $X_{3}=0$ as a perspective view. It contains the $\sigma_{22}$ and $\sigma_{12}$ (or $\sigma_{21}$ and $\sigma_{11}$ ) components at different levels $X_{3}$. At surface equilibrium $\quad \sigma_{33}=\sigma_{13}=\sigma_{23}=0 \quad$ and $\sigma_{12}\left(X_{3}\right)=\sigma_{21}\left(X_{3}\right)$. The surface stress is thus the excess

$s_{i j}^{(3)}=\int \sigma_{i j}\left(X_{3}\right) d X_{3} / \int d X_{3}-\sigma_{i j}\left(X_{3} \rightarrow-\infty\right)$

$(i, j=1,2)$. Obviously it is an intrinsic material property independent of the external stresses applied on the body.

Thus there is no interfacial excess of the $\sigma_{i 3}$ components. In contrast, at the interface $\left(\mathrm{x}_{3}=0\right)$ $\sigma_{i j}^{I} \neq \sigma_{i j}^{I I}(\mathrm{i}, \mathrm{j}=1,2)$. The interfacial stress components $\mathrm{s}_{\mathrm{ij}}$ are thus defined as the surface excess quantity of the components $\sigma_{i j}(\mathrm{i}, \mathrm{j}=1,2)$ of bulk stress tensor. Obviously $s_{i j}(\mathrm{i}, \mathrm{j}=1,2,3)$ with $\mathrm{s}_{\mathrm{i} 3}=0$ when relaxed in respect to surface $\mathrm{x}_{3}=0$. In figure $1 \mathrm{c}$ it can be seen that $\mathrm{s}_{\mathrm{ij}}$ at equilibrium is a degenerated $3 \mathrm{D}$, second rank tensor or $2 \mathrm{D}$ second rank tensor. On figure $1 \mathrm{~b}$ we sketch and precise surface stress excess at a vacuum-crystal interface.

\subsubsection{Surface strain}

Let us suppose that there is forced lattice coherence from bulk phase I to bulk phase II along $\mathrm{x}_{3}=0$. Then by definition of coherence at the interface there is $\varepsilon_{i 3}^{I}=\varepsilon_{3}^{I I}$ with $\mathrm{i}, \mathrm{j}=1,2$. Thus one only can define the interfacial strain tensor $e_{i j}$ as the excess of the components $\varepsilon_{i j}^{I}, \varepsilon_{i j}^{I I}$ of the bulk strain tensors. When one bulk phase is a fluid or vacuum, the solid near $\mathrm{x}_{3}=0$ presents an intrinsic excess of $\varepsilon_{\mathrm{i} 3}$ depending on the symmetry. It is called: surface strain. As surface stress, surface strain also is a symmetric second rank tensor $e_{i j}(i, j=1,2,3)$ with $e_{i j}=0($ if $i, j=1,2)$ (for the surface $\mathrm{x}_{3}=0$ ). In figure $1 \mathrm{c}$ we illustrate the complementary nature of surface stress and surface strain. Let us note as underlined by Nozières and Wolf $[9,10]$ that if generally the $\mathrm{e}_{33}$ component is an elastic relaxation it can also correspond to a mass transfer across the interface. It is important to notice that surface stress and surface strain being surface excesses, in the framework of linear bulk elasticity and at mechanical equilibrium, surface stress and 
surface strain are independent quantities. In other words there is no Hooke law for the surface [8]. Furthermore they are bulk stress and strain independent.

$$
\left[s_{i j}\right]=\left[\begin{array}{ccc}
s_{11} & s_{12} & 0 \\
s_{12} & s_{22} & 0 \\
0 & 0 & 0
\end{array}\right] ; \quad\left[e_{i 3}\right]=\left[\begin{array}{ccc}
0 & 0 & e_{13} \\
0 & 0 & e_{23} \\
e_{13} & e_{23} & e_{33}
\end{array}\right]
$$

Figure 1c: Illustration of the complementary nature for a given face (3) of the two intrinsic surface properties, sur(inter)face stress and strain at mechanical equilibrium.

\subsubsection{Surface elastic energy}

The surface (resp. interface) elastic energy can be defined as the work done to deform the surface $n$ (resp. interface). It simply reads for the interface $n=3[9,10]$, $W_{e l}^{\text {Surf. }}=\int\left(\sigma_{i 3} e_{i 3}^{n}+s_{i j}^{n} \varepsilon_{i j}\right) d S_{n}$ with $\mathrm{i}, \mathrm{j}=1,2,3$.

When the bulk phase I is vacuum and the phase II a solid, the mechanical equilibrium (1) gives at the surface $\mathrm{x}_{3}=0, \sigma_{i 3}^{I I}=\sigma_{i 3}^{I}=0$. Then the surface elastic energy reduces to

$W_{e l .}^{\text {Surf. }}=\int s_{i j}^{n} \varepsilon_{i j} d S_{n}$

The integral is performed over the non-deformed surface.

In fact (9) is the original definition of surface stress of Gibbs [6]. The surface stress $s_{i j}^{n}$ of a face $n$ is the work done to deform the surface $n$ at a constant number of atoms. Surface stress thus must not be confused with the surface energy $\gamma_{n}$ (the energy of creating a surface without deformation) of the face $n$, which is the work done to create this surface at constant strain. In fact for a free crystalline face there is [7]

$$
s_{i j}^{n}=\frac{\partial\left(\gamma_{n} S_{n}\right)}{\partial \varepsilon_{i j}}=\gamma_{n} \frac{\partial S_{n}}{\partial \varepsilon_{i j}}+S_{n} \frac{\partial \gamma_{n}}{\partial \varepsilon_{i j}}=\gamma_{n} \delta_{i j}+\frac{\partial \gamma_{n}}{\partial \varepsilon_{i j}}
$$

where $\delta_{\mathrm{ij}}$ is the Kronecker symbol. Relation (10) known as Shuttleworth relation [7] shows that for a liquid (where a surface cannot be deformed at a constant number of atoms so that $\partial \gamma_{n} / \partial \varepsilon_{i j}=0$ ) surface stress and surface energy are numerically equal (see more complete discussion in [104]). In order to recall the physical difference between surface stress and surface energy it is common to express $s_{i j}^{n}$ as a force per unit length and $\gamma_{\mathrm{n}}$ as energy per unit area. Notice that these two quantities have the same magnitude $\left(0.5 \mathrm{Jm}^{-2}\right)$. However $\gamma_{\mathrm{n}}$ is always a positive quantity $s_{i j}^{n}$ can be positive (tensile component) or negative (compressive component). 
In the following for the sake of simplicity we will mostly consider isotropic surfaces (symmetry greater than binary axis) where the surface stress tensor $\left[\mathrm{s}^{\mathrm{n}}\right]$ reduces to a scalar $\mathrm{s}^{\mathrm{n}}$ for a given orientation $\mathrm{n}$ but nevertheless varies with orientation in a cubic crystal. Only in amorphous materials $\mathrm{s}$ and $\gamma$ are direction $\mathrm{n}$-independent.

\subsection{Elastic description of real surfaces}

It is well known that surface defects such as adatoms or steps change the surface energy. For instance, foreign adsorption decreases the surface energy $\gamma$ by a quantity $\partial \gamma / \partial \mu=-\Gamma$ where $\mu$ is the chemical potential of the adsorbed species and $\Gamma$ the adsorption density [6]. On the other hand the surface energy of a vicinal surface (angle $\alpha$ ) can be written $\gamma=\gamma_{o}+\beta_{1} p+\beta_{2} p^{3}$ where $\gamma_{\mathrm{o}}$ is the surface energy of the terrace (reference face with a cusp in the $\gamma$-plot), $\mathrm{p}=\operatorname{tg} \alpha$ the macroscopic slope of the vicinal face and $\beta_{\mathrm{i}}$ some coefficients depending on step energy $\left(\beta_{1}\right)$ and step-step interaction $\left(\beta_{2}\right)$ (for a review see [21]).

In fact adatoms and steps also create a strain field in the otherwise flat surface and the underlying bulk substrate as well. Thus a complete elastic description of crystalline surfaces must include an elastic description of the defects. Indeed we will see (section 3.) that such elastic defects may have dramatic effects on crystal growth mechanisms.

\subsubsection{Elastic description of adatoms}

In 3D elasticity it is well known $[22,13,14]$ that the field of elastic strain far away a bulk defect located at $\mathrm{x}=\mathrm{x}^{(\mathrm{i})}$ can in the solid be calculated by considering the action at $\mathrm{x}>\mathrm{x}^{(\mathrm{i})}$ of a point force $\mathrm{F}\left(\mathrm{x}-\mathrm{x}^{(\mathrm{i})}\right)$ located at $\mathrm{x}=\mathrm{x}^{(\mathrm{i})}$. At mechanical equilibrium these forces reduce (up to the first order) to a localised force doublet. Its components can thus be written:

$$
F_{\alpha}\left(x-x^{(i)}\right)=A_{\alpha \beta} \frac{\partial}{\partial x_{\beta}} \delta\left(x-x^{(i)}\right)
$$

where $\delta(\mathrm{x})$ is the $3 \mathrm{D}$ dirac function, $(\alpha, \beta=1,2,3)$ and $A_{\alpha \beta}=\sum_{j} f_{\alpha, j} x_{\beta, j}^{(i)}$ a 3D tensor with $f_{\alpha, j}$ the $\alpha^{\text {th }}$ force component in between the defect $\mathrm{i}$ and atoms $\mathrm{j} \vec{x}_{j}^{(i)}$ (components: $x_{\beta j}^{(i)}$ ) apart. The point force at (i) (11) produces in the planar solid a displacement field whose components $u_{\alpha}^{(i)}$ are $[9,13,23]$ :

$u_{\alpha}^{(i)}=-\sum_{\beta} \int D_{\alpha \beta}\left(x^{\prime}, x\right) F_{\beta}\left(x-x^{(i)}\right) d x^{3}$

where $\mathrm{D}_{\alpha \beta}\left(\mathrm{x}^{\prime}, \mathrm{x}\right)$ is the $3 \mathrm{D}$ Green tensor given for isotropic elastic bodies in classical papers $[13,22]$ when (i) is located at the surface [13] and given in [22] when located in the bulk.

In a seminal paper Marchenko and Parshin [24] following Lau and Kohn [25] consider adatoms as true 2D elastic surface defects and thus model them by $2 \mathrm{D}$ point forces doublets. In other words they use relations (11) and (12) but only with $\alpha, \beta=1,2[24,25]$ and use the 2D Green tensor [13] to calculate elastic displacements in the plane of the surface. In fact, as depicted by Kern and Krohn [26], adatoms produce a force distribution around them and thus distort also the underlying bulk substrate. Adatoms thus must be described as 3D defects $(\alpha, \beta=1,2,3$ in (11) and (12)) and the displacement field calculated by means of 3D Green tensor [22]. The 3D dipole can only be reduced to a 2D one when the normal component of 
the force exerted by the adatom onto its underlying substrate can be neglected (see such a specific case in appendix I).

\subsubsection{Elastic description of steps}

* Step on a stress free body:

As an adatom, a step is not a true surface defect and thus can be described as a row of 3D (and not 2D) dipoles. In the following we assume the steps (located at $\mathrm{x}_{1}=0$ ) parallel to the direction $\mathrm{x}_{2}$, furthermore we assume that the steps are infinite in this direction. Thus the point forces describing the displacement field due to a step can be generally written

$$
\left\{\begin{array}{l}
F_{1}=A_{11} \frac{\partial}{\partial x_{1}} \delta(r)+A_{13} \frac{\partial}{\partial x_{3}} \delta(r) \\
F_{3}=A_{31} \frac{\partial}{\partial x_{1}} \delta(r)+A_{33} \frac{\partial}{\partial x_{3}} \delta(r)
\end{array}\right.
$$

Nevertheless as shown by Marchenko and Parshin [24], Andreev and Kosevich [8] and Nozières [27] a step has a non-vanishing moment. Indeed since a step divides the surface in two equivalent terraces, it is submitted to the surface stress $s$ of the two neighbouring terraces which exert a torque of moment sh (h being the height of the step) and thus tends to twist the crystal.

Obviously the force distribution (13) must restore the torque. For a high symmetry surface $\left(\mathrm{s}_{\mathrm{ij}}=\mathrm{s} \delta_{\mathrm{ij}}\right)$, mechanical equilibrium conditions then lead to $A_{31}-A_{13}=s h$ where $\mathrm{h}$ is the height of the step.

Notice that usually, following Marchenko et al. [24] and Andreev et al. [8] steps are described in a simpler way by defining a single force dipole $F_{1}$ in the direction $x_{1}$ completed by a vertical force dipole $\mathrm{F}_{3}$ restoring the moment sh. The point force distribution describing the step thus reads:

$$
\left\{\begin{array}{l}
F_{1}=A_{11} \frac{\partial}{\partial x_{1}} \delta(r) \\
\left.F_{3}=A_{31} \frac{\partial}{\partial x_{1}} \delta(r)\right)
\end{array}\right.
$$

with $A_{31}=s h$. Such a notation is justified in [26] (see section 3.1. of [26])

It is important to notice that when a step separates two domains of different surface stress $s_{1}$ and $s_{2}$ (it is for example the case of $\operatorname{Si}(100)$ face where monoatomic steps separate $(2 \times 1)$ and (1x2) reconstructed surfaces) the point forces distribution that describes the step must include a non-vanishing resulting force $\left(\mathrm{s}_{1}-\mathrm{s}_{2}\right)$ near the step before relaxation. In this case an elastic monopole has to be added and thus (14) becomes

$$
\left\{\begin{array}{c}
F_{1}=\left(s_{1}-s_{2}\right) \delta(x)+A_{11} \frac{\partial}{\partial x_{1}} \delta(r) \\
\left.F_{3}=A_{31} \frac{\partial}{\partial x_{1}} \delta(r)\right)
\end{array}\right.
$$


Obviously $\mathrm{A}_{11}$ and $\mathrm{A}_{31}$ values can only be obtained from atomistic calculations.

* Step on a stressed body:

Last but not least let us note that until now we have only considered elastic description of a step on a crystal free of any bulk stress. However there is an additional effect at the surface of a bulk stressed solid (or a non relaxed epitaxial layer). Indeed in this case there is a discontinuity of bulk stress in the surface height and the lateral force on one side of the solid is not compensated by an equal force on the other side. The result once more is a supplementary force monopole located at the steps. The intensity of this force naturally is the bulk stress $\sigma$ (see 1.1.) times the step height $h$.

Generally for such stressed solids the dipolar contribution can be neglected (upper order) and thus the step before relaxation is described by the following point force distribution:

$F_{1}=\sigma h \delta(x)$

\subsubsection{Elastic interaction of elastic defects}

Since surface defects create a displacement field in the underlying substrate, surface defects interact by way of the underlying deformation. The interaction energy between two defects is simply the work done by the force distribution $f_{\alpha}^{(1)}$ of the first defect due to the displacement field $u_{\alpha}^{(2)}$ generated by the other defect. It reads [13,14,23]

$$
U^{\mathrm{int} .}=\frac{1}{2} \int f_{\alpha}^{(1)}\left(x-x^{(i)}\right) u_{\alpha}^{(2)}(x) d V+\frac{1}{2} \int f_{\alpha}^{(2)}\left(x-x^{(i)}\right) u_{\alpha}^{(1)}(x) d V
$$

A lot of literature on elastic interactions between point defects exists for many situations. For defects at the surface Rickman and Srolovitz have proposed a generalised approach [28]. Their results are summarized on table I (in absence of normal forces $F_{3}$ ) where each surface defect is characterized by its dimension (D) and its multipole order (m). An adatom thus is characterized by $\mathrm{D}=0$ and $\mathrm{m}=1$ (dipole), whereas a step $(\mathrm{D}=1)$ can be characterized by $\mathrm{m}=1$ (dipoles) or $\mathrm{m}=0$ (monopoles) according to the nature of the step (step on a stress free surface or boundary in between two stressed domains) (see section 1.3.2. and figure 2).

a) 000000000000000

$$
000000000000000
$$

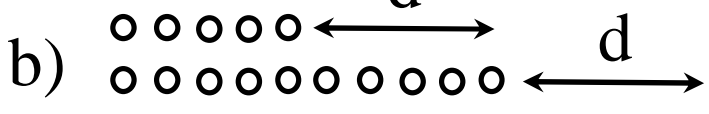
000000000000000

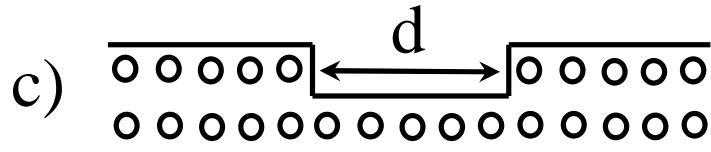

Figure 2: Elastic interaction in between:

a/ 2 adatoms considered as elastic dipoles $(D=0, m=1)$,

b/ 2 steps bearing dipoles $(D=1, m=1)$ of same sign or (and) monopoles $(D=1, m=0)$ of same sign when the solid is stressed;

c/ 2 steps of opposite sign. 


\begin{tabular}{|c|c|c|c|}
\hline & $\begin{array}{c}\text { Adatom } \\
\text { dipole } \\
(\mathrm{D}=0, \mathrm{~m}=1)\end{array}$ & $\begin{array}{c}\text { Step } \\
\text { on a stress free body } \\
\text { line of dipoles } \\
(\mathrm{D}=1, \mathrm{~m}=1)\end{array}$ & $\begin{array}{c}\text { Step } \\
\text { on a stressed body } \\
\text { line of monopoles or } \\
\text { (semi infinite sheet of } \\
\text { dipoles) } \\
(\mathrm{D}=1, \mathrm{~m}=0)\end{array}$ \\
\hline $\begin{array}{c}\text { Adatom } \\
\text { dipole } \\
(\mathrm{D}=0, \mathrm{~m}=1)\end{array}$ & $\begin{array}{c}+\mathrm{d}^{-3} \\
\text { (repulsion if same sign) }\end{array}$ & $\begin{array}{c}+\mathrm{d}^{-2} \\
\text { (repulsion if same sign) }\end{array}$ & $+x^{-1}$ \\
\hline $\begin{array}{c}\text { Step } \\
\text { on a stress free body } \\
\text { line of dipoles } \\
(\mathrm{D}=1, \mathrm{~m}=1)\end{array}$ & $d^{-2}$ & $\begin{array}{c}\mathrm{d}^{-2} \\
\text { (repulsion if same sign) }\end{array}$ & \\
\hline $\begin{array}{c}\text { Step } \\
\text { on a stressed body } \\
\text { line of monopoles or } \\
\text { (semi infinite sheet of } \\
\text { dipoles) } \\
(\mathrm{D}=1, \mathrm{~m}=0)\end{array}$ & $+x^{-1}$ & -- & $\begin{array}{l}\ln (\mathrm{d}) \\
\text { (attraction if same sign) }\end{array}$ \\
\hline
\end{tabular}

Table I: Elastic interaction dependence versus the distance $d>0$ or the abcissa $-\infty<x<+\infty$ in between some elastic defects when the normal force $F_{3}$ is neglected.

The so obtained results are easy to memorize if one admits the repulsive law $\mathrm{d}^{-3}$ between similar adatoms (see figure 2a). The interaction between a line of dipoles (step) and a dipole is thus simply obtained by integration along the line and leads to an interaction in $\mathrm{d}^{-2}$. (The elastic interaction between two steps on a stress free body (see figures $2 b$ and $2 c$ ) is obtained by integration over the step of infinite length of the $\mathrm{d}^{-3}$ law then multiplied by the number of dipoles in the other step giving again $\mathrm{a} \mathrm{d}^{-2}$ law). The interaction of adatoms (dipoles) with step of a stressed body (the step bears monopoles) gives an $\mathrm{x}^{-1}$ law interaction that means interaction changes sign with abscissa $\mathrm{x}$. (Nevertheless the definite sign of the interaction depends upon the respective signs of monopoles and dipoles). This result is equivalent to the interaction of a dipole and a semi-infinite sheet of dipoles. Indeed as in electrostatics a semiinfinite sheet of dipoles is equivalent to a distribution of monopoles located at the border of the sheet. Thus the interaction between a dipole and a step on a stressed body can thus be obtained by a supplementary integration of the $\mathrm{d}^{-2}$ law over the various rows of dipoles constituting the domain and thus give $\mathrm{a}^{-1}$ interaction law.

At last the interaction between two parallel steps on a stressed body (two lines of monopoles of same sign. (Fig $2 b)$ ) gives an interaction law $+\ln (d)$. This attractive interaction can be also obtained from the electrostatic equivalence from a new integration of the $\mathrm{x}^{-1}$ law over the various rows of dipoles. Such steps of opposite sign (fig $2 \mathrm{c}$ ) give a repulsive interaction $-\ln (d)$.

From these results it is easy to see that the interaction between an adatom and a stressed island of lateral size $\mathrm{L}$ must vary from $\mathrm{d}^{-1}$ for large $\mathrm{L}$ (interaction between an adatom and a sheet of dipoles) to $\mathrm{d}^{-2}$ for weak $\mathrm{L}$ (interaction in between adatoms and a row of dipoles). Obviously the intensity of the interaction depends upon the detail of the calculation of the coefficients $A_{\alpha \beta}$ but does not change nature and sign. 
In fact, for completeness we stress the fact that all these classical works $[8,24,26,28]$ summarized in table I only hold for semi-infinite isotropic substrates where Green's function formalism applies. This is no more the case for cubic crystals [29,30]. On the (001) or (111) faces of these crystals the interaction energy of identical adatoms become very anisotropic even with change of sign, however interaction of dipole rows remain with the same sign whatever their azimuth. More recently Peyla et al [31,32] showed that for very thin substrates things change too. For example identical adatoms deposited onto a true 2D isotropic layer may attract or repel each other according to the in-plane direction. The local force distribution seems to be responsible. For thicker sheets this effect goes backwards to usual $\mathrm{d}^{-3}$ repulsion valid for thick isotropic substrates.

\subsection{Morphology and surface stability of a stressed body}

A stressed body may become unstable against undulations or spontaneous formation of stressed domains. In this section we will only give some semi quantitative arguments for a better understanding of basic phenomena leading to such instabilities. In connection with growth mechanism we will examine another instability, the step bunching in section 4.3.

\subsubsection{Asaro-Tiller-Grienfeld (ATG) instability}

Since Asaro and Tiller [33] then very later Grienfeld [34,35] it is known that a planar surface of a stressed solid is unstable against undulation. There is an abundant theoretical [33$41]$ and some experimental facts $[42,43]$ on this instability. In the framework of these lectures we will follow $[33,36,39]$ most simplest arguments. For this purpose we consider the free energy change $\Delta \mathrm{F}$ per unit area induced by a periodic one-dimensional surface undulation $z(x)=h \cos (\omega x)$ of the surface of a uniaxially in-plane stressed solid $\sigma=\sigma_{\mathrm{xx}}$ ( $\omega$ is the wave vector $\omega=2 \pi / \lambda, \mathrm{h}>0$ the amplitude of the undulation, Oz pointing in the solid) (see figure 3 ).

This free energy change per unit area contains two terms. The first one $\Delta \mathrm{F}_{1}$ is the capillary energy change due to surface area increase (surface energy $\gamma$ being isotropic):

$$
\Delta F_{1}=F_{1}^{\text {und. }}-F_{1}^{\text {flat }}=\gamma \frac{\iint\left\{\sqrt{1+\left(\frac{\partial z}{\partial x}\right)^{2}}-1\right\} d x d y}{\iint d x d y} \approx \frac{\gamma}{2} \frac{\iint\left(\frac{\partial z}{\partial x}\right)^{2} d x d y}{\iint d x d y}=\frac{\gamma}{4} h^{2} \omega^{2}
$$

where the last expression is valid for small slope $\partial z / \partial x<1$ or $h / \lambda<<1$

The second term $\Delta \mathrm{F}_{2}$ is the elastic energy change per unit area induced by the undulation. Even for isotropic elasticity it is difficult to calculate since it depends upon the detail of the elastic relaxation. We follow the original paper [36] with a simplified version of first order in $h / \lambda$ where the approximation (trick) is to apply sinusoidal forces along a flat surface (The undulation is treated as a surface defect on a planar semi-infinite surface). In fact along a flat surface at $\mathrm{z}=0$, as well as in the bulk $(\mathrm{z}>0)$, there is in the solid an in-plane constant stress $\sigma_{x x}(x, z)=\sigma$ so that nowhere $f_{x}$ forces apply, gravity or other so-called body forces being excluded. But when some 1D undulation $\mathrm{z}(\mathrm{x})$ of some arbitrary amplitude $\mathrm{h}>0$ at the surface and $\omega=2 \pi / \lambda$ is created (fig. 3 ), there develops at the surface some excess forces. When the amplitude is small in respect to $\lambda,(h / \lambda<<1)$ there may tentatively be a force density 
$f_{x}(x, 0)=\frac{d}{d x}(\sigma z) \approx \sigma \frac{d z}{d x}$, with $f_{y}(x, 0)=0$ of course and a normal force $f_{z}(x, 0)$ close to zero at second order of the slope $\mathrm{dz} / \mathrm{dx}$.
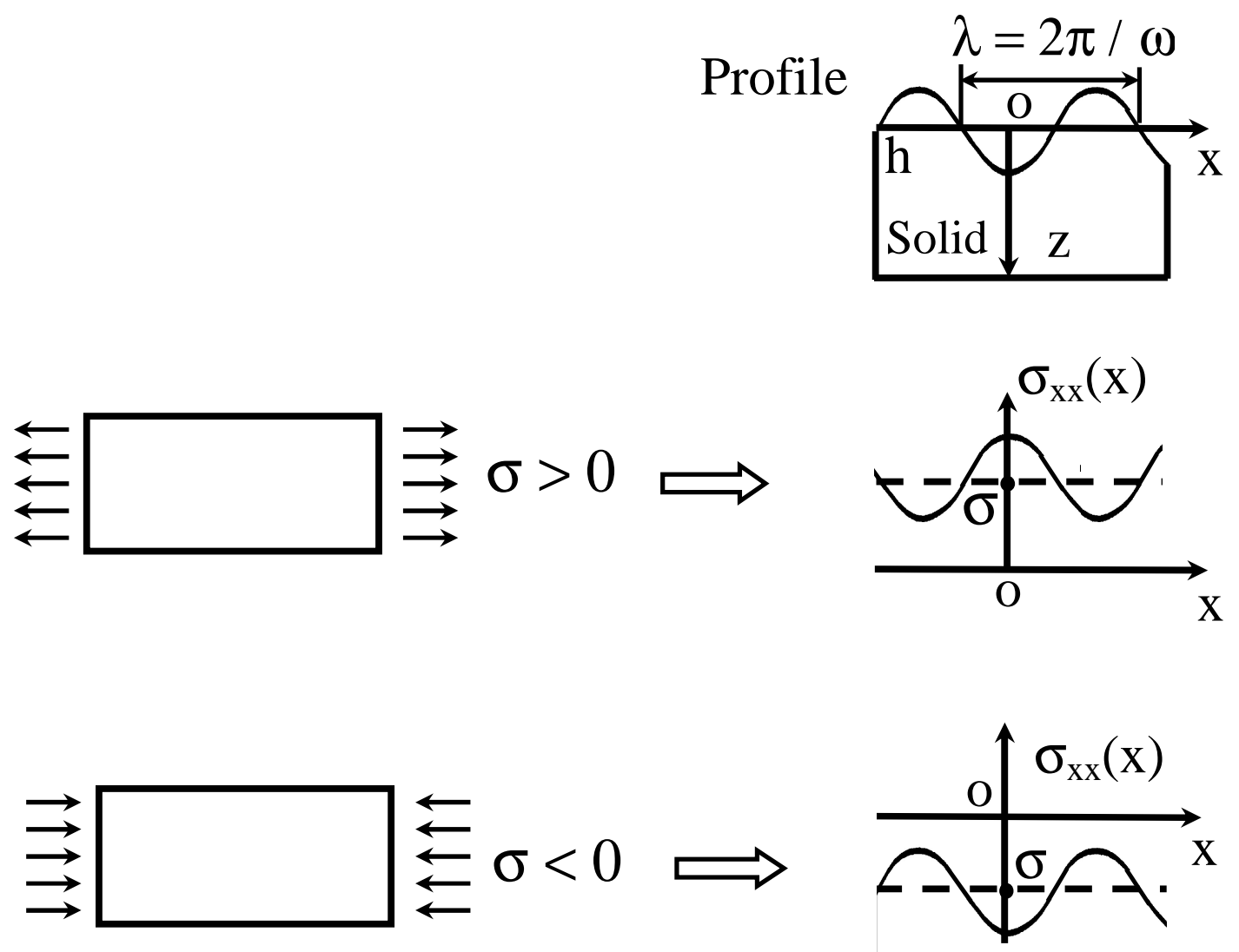

Figure 3: Azaro-Tiller-Grienfeld instability of a planar surface under an external uniaxially stress $\sigma_{\|}=\sigma$. When the surface develops a $1 D$ sinusoidal undulation hcos $\omega x, h>0, O z$ pointing in the bulk, the surface at first order develops a stress concentration either tensile $(\sigma>0)$ or compressive $(\sigma<0)$ in the valleys.

Let us suppose that such a line of forces $f_{x}(x, 0)$ is applied on the surface of a planar semiinfinite isotropic solid, according to Love [14] the stress in excess of $\sigma$ is given by:

$\sigma_{x x}(x, 0)-\sigma=-\frac{2}{\pi} \int_{-\infty}^{+\infty} \frac{f_{x}\left(x^{\prime}, 0\right)}{x^{\prime}-x} d x^{\prime}=\frac{2 \sigma h \omega}{\pi} \int \frac{\sin \omega x^{\prime}}{x^{\prime}-x} d x^{\prime}$

According to [36] and appendix B the principal value of this Cauchy integral is $\pi \cos \omega \mathrm{x}$ so that

$$
\left(\sigma_{x x}(x, 0)-\sigma\right) / \sigma=2 \omega z(x)+\mathrm{O}\left[(h / \lambda)^{2}\right] ; \sigma_{y y}(x, 0)=0
$$

Thus at the surface an excess modulation of the in-plane stress appears. In figure 3 we observe for $\sigma>0$ that valleys bear maximum tensile stress, in figure 3 for $\sigma<0$ they bear maximum compressive stress. Crest at contrary, have smaller stress than the mean value $\sigma$, which is also the bulk value whatever tensile or compressive. 
Hooke's law under plane strain ( $\varepsilon_{y y}=0$ when the solid is infinite along Oy), at the surface $\mathrm{z}=0$, associates to the in-plane stress $\mathrm{xx}$ the only in-plane strain $\mathrm{xx}$ modulation from (19):

$$
\varepsilon_{x x}(x)=\frac{1-v^{2}}{E} \sigma_{x x}(x)=\varepsilon_{/ /}(1+2 \omega z(x))
$$

It follows the strain modulation around the mean in-plane strain $\varepsilon_{/ /}=\frac{1-v^{2}}{E} \sigma$. Due to Poisson's effect the normal strain modulates with opposite phase $\varepsilon_{z z}(x)=-\frac{v}{1-v} \varepsilon_{x x}(x)$. Along the undulated surface the lattice parameters vary as $a_{/ /}(x)=a_{o}\left(1+\varepsilon_{x x}(x)\right)$, $a_{\perp}(x)=a_{o}\left(1+\varepsilon_{z z}(x)\right)$ where $\mathrm{a}_{\mathrm{o}}$ is the parameter of the stress free crystal. In the valleys $\mathrm{x}=\mathrm{n} \lambda$ (fig 3): $\left[a_{/ /}(n \lambda)-a_{o}\right] / a_{o}=\varepsilon_{/ /}(1+4 \pi h / \lambda)$ there is strain concentration whereas on the crests $\mathrm{x}=(2 \mathrm{n}+1) \lambda:\left[a_{/ /}((2 n+1) \lambda)-a_{o}\right] / a_{o}=\varepsilon_{/ /}(1-4 \pi h / \lambda)$ there is strain deficit. One says that the crests relax.

Let us note that the strain-stress modulations penetrate in the bulk $\mathrm{z}>0$. The more general Green's kernel $\operatorname{Re}\left[\frac{x^{\prime}-x}{\left(x^{\prime}-z\right)^{2}}\right]$ has to be used. The result for $\mathrm{z}>0$ of (19) is then valid when its right side is multiplied by $(1-\pi z / \lambda) \exp (-2 \pi z / \lambda)$ showing that the in-plane stress damps exponentially with a decay distance $\lambda / 2 \pi$ independent of the amplitude $\mathrm{h}$ (at this degree of approximation). This result and others for $\sigma_{\mathrm{yy}}, \sigma_{\mathrm{xz}}$ have been successively obtained by different methods by $[33,34,36,39]$.

The matter is now to calculate the strain energy per unit area, of the undulated surface, that means the integral (4) $F_{2}^{u n d}=\frac{1}{2} \int_{V} \sigma_{i k} \varepsilon_{i k} d V / \int_{S} d S$ extended over all the semi-infinite solid z $>0$. This would be a cumbersome task since in the bulk Hookes'law gives several $\varepsilon_{\mathrm{ik}}$ components for each $\sigma_{\mathrm{ik}}$. Fortunately Marchenko [44] showed how to reduce this volume integral in a surface integral where then the $\varepsilon_{\mathrm{xx}}$ component is connected to one $\sigma_{\mathrm{xx}}$ component (see appendix C) so that

$$
F_{2}^{\text {und }}=\frac{1}{2} \int_{S} f_{i} u_{i} d S / \int_{S} d S
$$

where $f_{i}$ are the surface force density components inducing the surface displacement components $\mathrm{u}_{\mathrm{i}}$. Since on the slightly undulated surface exists only, at first order, the $f_{x}(x, 0)=2 \sigma \omega(d z / d x)$ component and the corresponding in-plane displacement from (20) $u_{x}(x, 0)=\int \varepsilon_{x x}(x, 0) d x=\frac{1-v^{2}}{E} \sigma[x+2 h \sin \omega x]$ where we put $u_{x}(x, 0)=0$ by convention. From (21) with these functions: $F_{2}^{\text {und }}=-4\left(1-v^{2}\right) \sigma^{2} h^{2} \omega / E$ so that for the flat surface $F_{2}^{\text {flat }}=0$ and 
$\Delta F_{2}=F_{2}^{\text {und }}-F_{2}^{\text {flat }}=-4\left(1-v^{2}\right) \sigma^{2} h^{2} \omega / E$

We learn that the elastic energy density drops when an undulation develop on a in-plane $\sigma_{x x}=\sigma$ stressed solid irrespective stress is tensile or compressive. To this opposes the surface energy change (18) so that the total free energy change is

$$
\Delta F=\Delta F_{1}+\Delta F_{2}=h^{2} \omega\left[\frac{\gamma}{2} \omega-\frac{4\left(1-v^{2}\right)}{E} \sigma^{2}\right]
$$

Instability occurs when $\Delta \mathrm{F}<0$ that means the applied stress, tensile or compressive, overpasses the critical value

$$
|\sigma| \geq\left[\frac{E \gamma \omega}{8\left(1-v^{2}\right)}\right]^{1 / 2}
$$

In terms of critical strain (20) and wavelength (24) gives $\varepsilon_{/ /} \geq\left[\frac{\pi\left(1-v^{2}\right) \gamma}{4 E \lambda}\right]^{1 / 2}$. Since $\gamma / \mathrm{E}$ values of a wide variety of materials [45] are among $10^{-2} \mathrm{~nm}$, for usual strain $\varepsilon_{/ /}=10^{-2}$ as e.g. epitaxial strain, $\lambda \approx 10^{2} \mathrm{~nm}$ so that nanoscopic undulations may develop. The early time evolution of the surface profile is driven by the chemical potential gradient along the surface $[33,38]$.

Obviously a more complete treatment must contain first real anisotropy $\gamma$ behaviour, then surface stress and gravity as well. The surface stress effect on the instability has been studied by Grilhe [46] and $\mathrm{Wu}$ [44]. An interesting result [46] is that the symmetry between compression and tension is broken by surface stress since the $\sigma^{2}$ dependence adds a $\sigma$ contribution. If $(s-\gamma)=\partial \gamma / \partial \varepsilon$ has same sign as the bulk stress $\sigma$ the critical wavelength is reduced. When introducing gravity [27], a new stabilizing term may appear in $\Delta \mathrm{F}$ when acting in a proper direction. The effective contribution to instability would start at sizes $h$ of the order of the millimeter. More precisely the ATG instability is thus in between two critical wavelengths. Only a non-linear analysis can give the true final shape [48]

Let us note that Spencer et al. [40,41] have studied the case of an epitaxial stressed layer on a lattice mismatched substrate but the same limitation of constant $\gamma$ are used. The free surface also is unstable in respect to a sufficiently long wavelength but the critical wavelength now depends upon the thickness $d$ of the strained film in the very special case the substrate is infinitely stiff. Such undulations have been interpretated as a possible origin of the Stranski Krastanov transition on kinked faces (diffuse faces) [35]. For F faces (singular faces) the origin of Stranski-Krastanov transition will be discussed in section 3.2.3.3.

\subsubsection{Spontaneous formation of stressed domains}

Marchenko [44] was the first to propose that elastic interaction between surface antiphase domains may lead to periodic patterns due to alternating surface stress discontinuities $\pm \Delta \mathrm{s}$ (see figure 4a). A special case is for example the $\mathrm{Si}(100)$ surface that exhibits reconstructed $(1 \times 2)$ or $(2 \times 1)$ domains of surface stress $s_{1}$ and $s_{2}$ according to the level of the reconstructed terraces. Due to the anisotropy of the flat $\mathrm{Si}(100)$ surface, a vicinal $\mathrm{Si}(100)$ surface can be 
represented by a parallel line of alterning monopoles bearing the stress discontinuities $\Delta \mathrm{s}=\mathrm{s}_{1}$ $\mathrm{s}_{2}$ (see (15) and figure 4b). Alerhand et al [49] took this over for this case and made the balance between the domain boundary creation energy and the elastic interaction that leads to a selection of the size of the domains.

a)

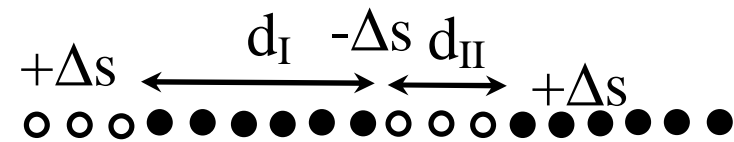

b)

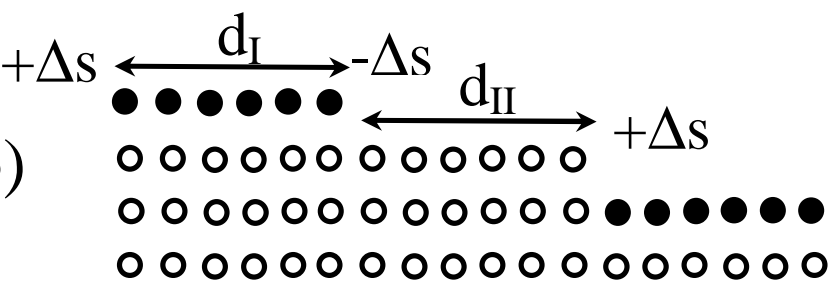

c)

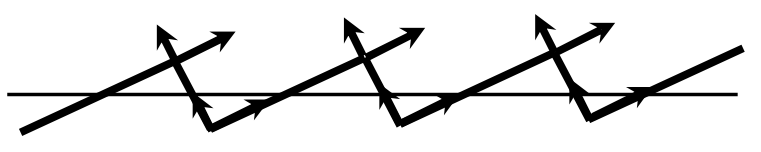

Figure 4:

a/ antiphase surface domains $(D=1, m=0)$ bearing surface stress discontinuities $\left(-\Delta s=s_{1}-s_{2}\right.$, $\left.+\Delta s=s_{1}+s_{2}\right)$;

b/ case of the (001) Si vicinal face similar to a;

c/ unstable flat face developing a vicinal of macrofacets, the arrows representing the noncompensated surface stress of the macro-facets.

More precisely for a surface with periodic (period $\mathrm{d}=\mathrm{d}_{\mathrm{I}}+\mathrm{d}_{\mathrm{II}}$ ) alternating domains of size $d_{I}$ and $d_{I I}$, the total energy per unit length reads [44,49] $U=\frac{\chi h}{d}-\frac{1-v^{2}}{\pi E} \Delta s^{2} \ln \left(\frac{d}{2 \pi a} \sin \pi \frac{d_{I}}{d}\right)$ where the first term is the boundary creation energy per unit length $(\chi)$ and the second term the elastic energy where one recognises the $\ln (d)$ dependence (see table I) and where the sinus comes from the periodicity. The minimisation of $\mathrm{U}$ in respect to $\mathrm{d}_{\mathrm{I}}$ gives the equilibrium size of each domain. Furthermore, stretching or compressing the crystal parallel to the surface and normally to the steps favours one type of domain over the other as found experimentally by Webb et al. [50]. More recently, B.Croset et al. [51] have completed Marchenko-Alerhand's theory by taking into account the elastic self-energy of each domains so that epitaxial strain somewhat relaxed by dislocations of Frenkel-Kontorova type could be considered. For completeness notice that the Marchenko elastic interaction is also at the basis of spontaneous periodic faceting of unstable crystal surfaces (see figure 4c). For a review see [52,53].

\section{MACROSCOPIC THERMODYNAMIC TREATMENT OF EPITAXIALLY STRAINED CRYSTALS}

\subsection{Wetting conditions versus elasticity}

Let us recall that three possible mechanisms of epitaxial growth of a crystal A onto a crystal B have been recognized: the 3D (or Volmer-Weber growth), the layer by layer (or Frank-van der Merwe growth) and the layer by layer growth followed by 3D growth (or Stranski 
Krastanov growth). In absence of misfit Bauer $[11,12,54]$ rationalised these growth modes by defining the so-called wetting factor

$$
\Phi_{\infty}=2 \gamma_{A}-\beta=\gamma_{A}+\gamma_{A B}-\gamma_{B}
$$

In (25) we also use the Dupré relation [55] $\beta=\gamma_{A}+\gamma_{B}-\gamma_{A B}$ with $\gamma_{B}$ the surface energy of $\mathrm{B}, \gamma_{\mathrm{AB}}$ the interfacial energy and $\beta$ the adhesion energy of $\mathrm{A}$ on $\mathrm{B}$. The wetting factor (25) in fact is connected to the capillary energy change $\Delta W_{\text {cap }}$ per unit area during the thermodynamical process in which a crystal A is created (volume $h \ell^{2}$ with 2 basal faces of energy $\gamma_{A}$ and 4 lateral faces of energy $\gamma_{A}$ for a parallelepiped crystal) then stuck on a substrate B (adhesion energy $-\beta$ ) (see figure 5a): $\Delta W_{\text {cap }}=\Phi_{\infty} \ell^{2}+4 \Phi^{\prime} h \ell$ where $\Phi^{\prime}=\gamma_{A}^{\prime}$

When $\Phi_{\infty}<0$ (more than perfect wetting) $\gamma_{A}+\gamma_{A B}<\gamma_{B}$ (or using Dupré relation $2 \gamma_{A}<\beta$ ) so that the minimum state of energy of the system is reached for an increasing interface that means a $2 \mathrm{D}$ film is more stable than a $3 \mathrm{D}$ crystal. When $\Phi_{\infty}>0$ (no perfect wetting) $\gamma_{A}+\gamma_{A B}>\gamma_{B}$ (or $2 \gamma_{A}>\beta$ ) so that the minimum state of energy is reached for a decreasing interface. A $3 \mathrm{D}$ crystal thus is more stable than a $2 \mathrm{D}$ film.

Notice that from this thermodynamical point of view 2D and 3D growth are thus well differentiated and cannot occur simultaneously. The case of Stranski Krastanov growth mode is more complex since obviously ${ }^{\S}$ the wetting energy must vary during the growth from negative values to positive values in such a manner $2 \mathrm{D}$ growth is relayed by $3 \mathrm{D}$ growth. Such dependence of wetting energy upon the number of deposited layers $\mathrm{z}$ can be justified by introducing long-range effect [56]. The wetting factor thus becomes $\Phi(z)=\Phi_{\infty}^{0} f(z)$ where $\mathrm{f}(\mathrm{z})$ is a decreasing function ${ }^{* *}$ of $\mathrm{z}$. We will come back to this point in section 2.2.2.

Naturally arises the question of the modification of the wetting factor (25) when there is a misfit and thus incidentally the modification of the growth mode by elasticity. For this purpose we just consider a new thermodynamical process analogous to the one we just described (figure 5a) but where now the crystal $\mathrm{A}$ is homogeneously strained before coherent accommodation onto a lattice mismatched substrate B (figure 5b). For the sake of simplicity we will consider a semi-infinite planar substrate B. The crystals are cubic of crystallographic parameter a (for A) and b (for B) with a (100) as contact plane both having parallel axis. We define the misfit strain as

$$
\varepsilon_{11}=\varepsilon_{22}=m_{o}=(b-a) / a
$$

\footnotetext{
${ }^{\S}$ In this paper we exclude growth mode change due to structural change during crystal growth.

** Let's mention that in the original paper of Stranski and Krastanov [57], ionic $\mathrm{NaCl}$ crystals have been considered so that normal to a (001) face there is an oscillating potential changing sign from a simple to a double layer.
} 
a)

$$
\mathrm{h}_{\mathbf{0}} \stackrel{\ell_{o} \gamma_{\mathrm{A}}}{\mathrm{W}_{\mathrm{A}} \mathrm{A}}
$$

B

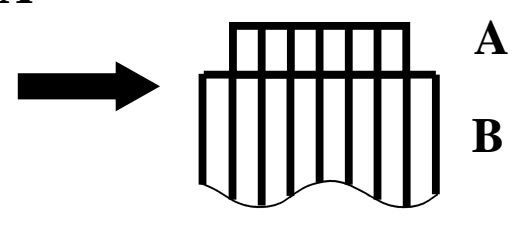

b)

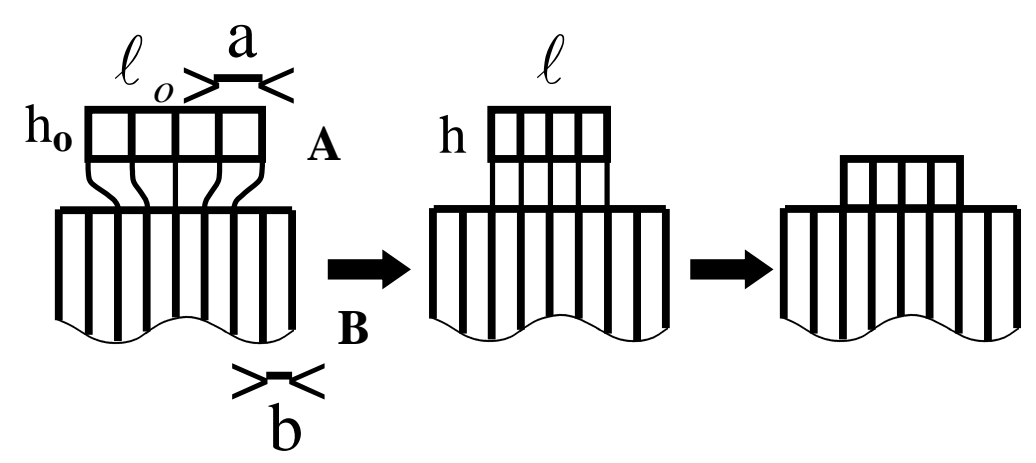

Figure 5: Schematic thermodynamic process of formation: a/ in absence of misfit the $3 D$ crystal $A$ is created then accommodated onto a isomorphous substrate $B . \boldsymbol{b} /$ In the presence of misfit the crystal A (parameter a) is homogeneously strained (from $V_{o}=h_{o} \ell_{o}^{2}$ to $V=h \ell^{2}$ ) before accommodation and adhesion onto the lattice mismatched substrate $B$ (parameter $b$ ).

The free energy change following the sequence (figure $5 \mathrm{~b}$ ) reads $\Delta W=\left[2 \gamma_{A} \ell_{o}^{2}+4 \gamma_{A}^{\prime} \ell_{o} h_{o}\right\rfloor+\left[2 s_{A}\left(\ell^{2}-\ell_{o}^{2}\right)+4 s_{A}^{\prime}\left(\ell h-\ell_{o} h_{o}\right)\right\rfloor-\beta \ell^{2}$ where the first term is the energy of formation of the surfaces of the non-deformed crystal $\left(V_{o}=h_{o} \ell_{o}^{2}\right)$, the second term is the surface elastic work against surfaces deformation (from $V_{o}$ to $V=h \ell^{2}$ ) (see (9)) and the last term the adhesion energy of the deformed crystal on the substrate. Then using $\ell=\ell_{o}\left(1+m_{o}\right)$ and $h=h_{o}\left(1-\frac{2 v}{1-v} m_{o}\right)$ one obtains $\Delta W=\Phi_{\infty}^{m 0} \ell^{2}+4 \Phi_{\infty}^{\prime} h \ell$ with up to the second order in $\mathrm{m}_{\mathrm{o}}$ :

$$
\Phi_{\infty}^{m_{0}}=2 \gamma_{A}-\beta+4 m_{0}\left(s_{A}-\gamma_{A}\right) \text { and } \Phi_{\infty}^{\prime}=\gamma_{A}^{\prime}+m_{o}\left(s_{A}^{\prime}-\gamma_{A}^{\prime}\right) \frac{1-3 v}{1-v} .
$$

$\Phi_{\infty}^{m_{o}}$ thus is a generalised wetting factor that replaces the usual wetting factor $\Phi_{\infty}(25)$ when surface stress acts [58].

Owing to the Shuttleworth relation (10), relation (27) is nothing other than the expansion of the wetting factor (25) up to the first order in strain when $\beta$ is the adhesion energy of the accommodated material A over B. 
For usual epitaxial material $\mathrm{m}_{\mathrm{O}}=10^{-2}$ and for clean surfaces $\mathrm{s}_{\mathrm{A}}$ and $\gamma_{\mathrm{A}}$ roughly have the same order of magnitude so that the corrective term $4 m_{0}\left(s_{A}-\gamma_{A}\right)$ to the wetting energy remains weak. However surface stress contributes more to the wetting condition the smaller the wetting factor (25) is and surface stress differs from surface energy. It is the case of $\mathrm{Si} / \mathrm{Ge}$ system $\left(\mathrm{m}_{\mathrm{o}} \approx+4 \%\right)$ where $\Phi_{\infty}^{0}=150 \mathrm{erg} \mathrm{cm}^{-2}$ whereas $\Phi_{\infty}^{m}=380 \mathrm{erg} \mathrm{cm}^{-2}$ [58]. However for such a case of $3 \mathrm{D}$ growth (since $\Phi_{\infty}^{0}>0$ ) the surface stress effect is overestimated. Indeed owing to the condition $\sigma_{\mathrm{iz}}=0,3 \mathrm{D}$ crystals must relax by their free faces and thus the bulk strain is lowered from homogeneous misfit $\mathrm{m}_{\mathrm{o}}$ to non-homogeneous residual values $\varepsilon\left(\mathrm{x}_{1}, \mathrm{x}_{2}, \mathrm{x}_{3}\right)$. Furthermore if both crystals remain coherent, the interfacial stress $\mathrm{s}_{\mathrm{AB}}$ also works during the relaxation from $\mathrm{m}_{\mathrm{o}}$ to $\varepsilon\left(\mathrm{x}_{1}, \mathrm{x}_{2}, \mathrm{x}_{3}=0\right)$. The new wetting factor in presence of elastic relaxation $\Phi_{m}$ is easy to write formally. However it cannot be calculated in a simple way since the residual strain tensor components depend upon the precise shape of the 3D crystal and have to be calculated using some mechanical model. Furthermore interfacial stress values are poorly known.

In fact we will only remember that in most usual cases surface and interfacial stress corrections to the wetting conditions remain weak.

\subsection{Equilibrium state versus elasticity}

\subsubsection{Description of an epitaxial model system}

Our purpose in this section is to seek for the thermodynamical and mechanical equilibrium state of $\mathrm{A} / \mathrm{B}$.

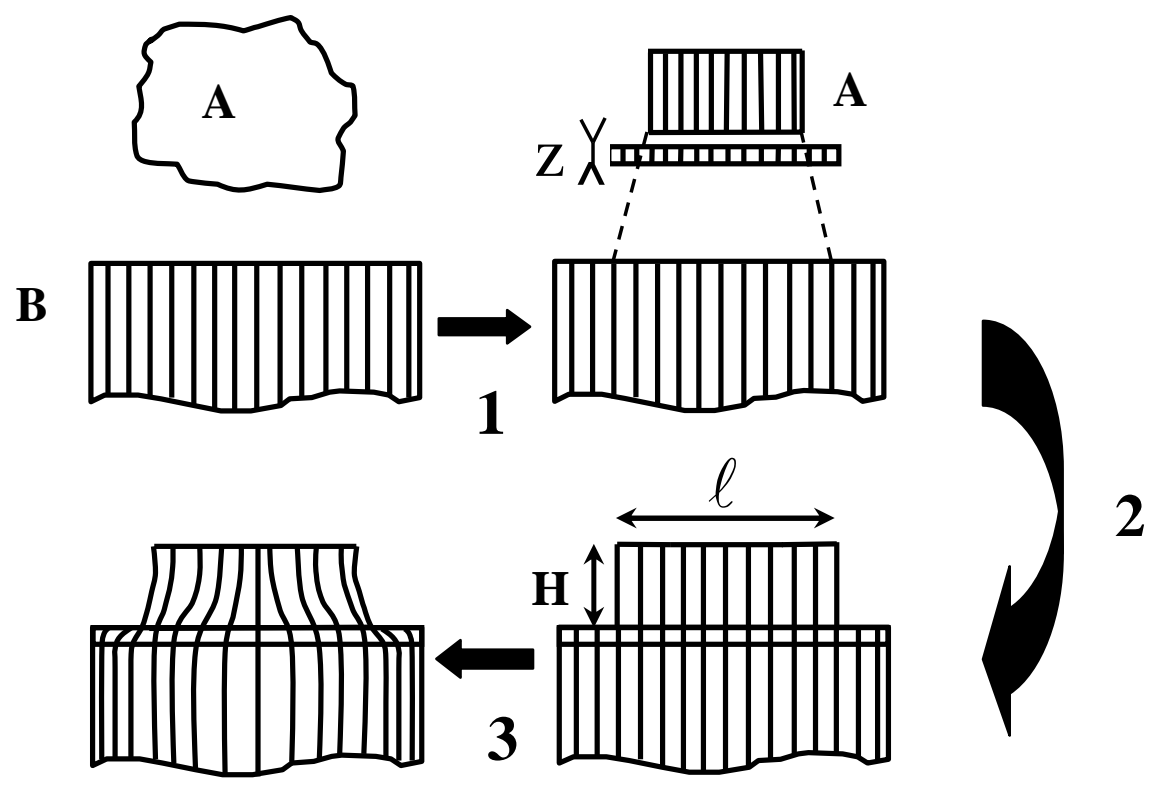

Figure 6: Schematic thermodynamic process of formation in the case of Stranski Krastanov growth. 1. formation, 2. homogeneous deformation for accommodation then adhesion, 3. inhomogeneous elastic relaxation. 
Therefore we will only consider a thermodynamic process where the deposit $\mathrm{A}$ is obtained from the condensation of a perfect vapour onto a lattice mismatched semi-infinite crystal. Furthermore both crystals A and B are cubic and supposed not to mix. The epitaxy is with parallel axis on a (001) plane. The (001) surfaces of A and B are supposed to be stable, that means each having a cusp in their surface energy plot $\gamma_{\mathrm{A}}$ and $\gamma_{\mathrm{B}}$. Furthermore the interface (001) has to be stable that means $\gamma_{\mathrm{AB}}>\mathrm{O}$ and having a cusp. The crystallographic parameters are $\mathrm{a}$ and $\mathrm{b}$ respectively, the in-plane misfit being given by (26).

We will consider the final state as a $3 D$ crystal of volume $V$ sitting on $z$ pseudomorphic layers over B that means Stranski Krastanov case (see figure 6). Indeed such formulation will allow discussing the two other cases of Volmer-Weber and Frank-van der Merwe growth as limiting cases for $\mathrm{z}=0$ and $\mathrm{V}=0$ respectively. Furthermore for the purpose of this lecture we will only consider box shaped 3D crystals. Other shapes will be discussed on the basis of some other papers [59-65]. Since as abovementioned surface stress generally plays a minor role on the wetting condition, in the following we will neglect surface stress and consider bulk elasticity only. We will come back to surface stress effects in section 3.2.3.4.

\subsubsection{Free energy change of the SK condensation}

Considering the condensation process described in figure 6 where 3D islands and 2D layers are formed from a vapour, the free energy change is composed of three terms:

$* \Delta \mathrm{F}_{1}$ is the chemical work to form (on an area $\mathrm{L}^{2}$ ) a $2 \mathrm{D}$ film of $\mathrm{z}$ layers and an island (volume $V=h \ell^{2}$ ) from an infinite reservoir of matter A defining the saturation pressure $\mathrm{P}_{\infty}$. It reads

$$
\Delta F_{1}=-\Delta \mu\left(z a L^{2}+h \ell^{2}\right)
$$

where $\Delta \mu=\frac{k T}{a^{3}} \ln \left(P / P_{\infty}\right)$ is called the supersaturation per unit volume of vapour $\mathrm{A}$ at pressure $P>P_{\infty}$ (supposed to be perfect) and a an atomic linear size.

$* \Delta \mathrm{F}_{2}$ corresponds to the formation of the surfaces of the crystal $\mathrm{A}$ followed by its adhesion on the bare substrate $\mathrm{B}$. It reads:

$\Delta F_{2}=\Phi_{\infty}\left[\left(L^{2}-\ell^{2}\right) f(z)+\ell^{2} f(z+h)\right]+4 \gamma_{A}^{\prime} h \ell$

where we consider the wetting energy $\Phi_{\infty}$ of (25) as being size dependent as mentioned in section 2.1. More precisely since surface and adhesion energies are excess quantities they are only well defined for semi-infinite solids. For a finite solid that only contains a few layers, surface and adhesion energy must depend on the number of layers $\mathrm{z}$ and thus read $\gamma_{\mathrm{A}}(\mathrm{z})$ and $\beta(\mathrm{z})$. The evolution with $\mathrm{z}$ by a decreasing function $\mathrm{f}(\mathrm{z})$ is quite usual in $2 \mathrm{D}$ multiplayer condensation (see [66]) or surface melting [67,68]. In the following we choose an exponential behaviour as used and justified for semi-conductors. The wetting energy now reads $[69,70]$ :

$$
\Phi(z)=\Phi_{\infty}(1-\exp (-z / \zeta))
$$

where $\zeta$ is a screening factor close to unity so that in the following we put $\zeta=1$. 
Thus $\Delta F_{2}$ reads:

$$
\Delta F_{2}=\Phi_{\infty}\left[\left(L^{2}-\ell^{2}\right)(1-\exp (-z))+\ell^{2}(1-\exp (-(z+h / a)))\right]+4 \gamma_{A}^{\prime} h \ell
$$

In the following since $\mathrm{z}+\mathrm{h} / \mathrm{a}>\mathrm{h} / \mathrm{a}$ and owing to the quick variation of the exponential we will neglect $\exp (-(z+h / a))$ against $\exp (-z)$. Notice again that we have neglected surface stress work.

$* \Delta \mathrm{F}_{3}$ is the total elastic energy stored by the composite system $\mathrm{A} / \mathrm{B}$. It can be written as the sum of the elastic energies stored in the crystal A and in the substrate B respectively and thus reads:

$$
\Delta F_{3}=\mathrm{E}_{o}\left[z a L^{2}+h \ell^{2} R(h, \ell)\right\rfloor
$$

where $\mathrm{E}_{o}=Y m_{o}^{2}$ where $\mathrm{Y}$ is a combination of elastic constants (see section 1.1.4.). For micro or nano crystals naturally arises the question of the validity of bulk elastic constants. Nevertheless it seems [71] that surface stress considerations avoid using size dependent elastic constants (see appendix D). The first term of relation (31), is the homogeneous energy stored by z pseudomorphous layers of thickness a and lateral size L. The second term is the elastic energy originating from the $3 \mathrm{D}$ upperlying crystal of volume $V=h \ell^{2}$. The factor $0<R(h, \ell)<1$ is a relaxation factor. Obviously in absence of $3 \mathrm{D}$ crystals $\mathrm{V}=0$, one recovers $\Delta F_{3}=\mathrm{E}_{o} z a L^{2}$ the elastic energy of the pseudomorphous film. Let us note that the relaxation factor $R(h, \ell)$ has to be calculated for each specific case. It originates from the fact that the normal stress components $\sigma_{\mathrm{iz}}$ along the free surface has to vanish at mechanical equilibrium. It has not the same expression in case of $3 D$ growth $(z=0)$, SK growth $(z \neq 0, V \neq 0)$ or $2 D$ growth where it does not play any role $(\mathrm{V}=0)$ or more exactly a minor role since $h=a$ and $r=a / \ell$.

For a 3D crystal sitting on a bare substrate B (Volmer-Weber growth) the epitaxial contact in between the $3 \mathrm{D}$ deposit and its lattice mismatched substrate is supposed to be coherent and to remain coherent during the elastic relaxation of the $3 \mathrm{D}$ crystal. In this case, during the relaxation the crystal A drags the atoms of the contact area and produces a strain field in the substrate B which was initially strain free. This created strain field may be calculated by using point forces [59,72] or more properly by using a self-consistent approach [73,74]. After relaxation the 3D crystal and its substrate are inhomogeneously strained (see figure 6). This means that although the total energy density has been lowered by elastic relaxation, the elastic energy density in the substrate has increased.

The Stranski-Krastanov case is more complicate. Indeed before relaxation, deposit A (3D crystal A and the z pseudomorphous layers) is homogeneously strained whereas the substrate $\mathrm{B}$ is stress free. After relaxation the elastic energy density in the $3 \mathrm{D}$ crystal as well as the elastic energy density in the underlying film have been lowered in respect to the initial pseudomorphous strained layers (see figure 6). If the number of 2D layers is weak enough the inhomogeneous strain field induced in the 2D layers by the relaxation of the 3D crystal penetrates into the underlying foreign substrate B. The elastic energy density in the substrate $\mathrm{B}$ thus increases in respect to the initial stress free lattice mismatched substrate (see figure 7). If on contrary the number of layers increases the positive contribution of B vanishes. In other words the strain fields, induced by the elastic relaxation of 3D islands are not the same for a 
bare substrate and a composite (2D A layers + B) substrate. In the former VW case, the elastic strain density can be analytically evaluated in a self-consistent fashion (see figure 7), but to our best knowledge it is not the case up to now for a composite substrate. So in the discussion we will distinguish the relaxation factor in case of $3 \mathrm{D}$ growth $R^{3 D}(h, \ell)$ and in case of Stranski Krastanov growth $R^{S K}(h, \ell, z)$.

Figure 7: Elastic energy relaxation factor $R^{3 D}(r)$ versus shape ratio $r=h / \ell$ for a $3 D$ box shaped crystal calculated from [74,87] when the deposit and the substrate have the same elastic properties. $R_{A}$ and $R_{B}$ are the relaxation factors of the deposit $A$ and substrate $B$ respectively. $R=R_{A}+R_{B}$. Multiplied by $\mathrm{E}_{o}=Y m_{o}^{2}, \quad Y=E /(1-v)$ each gives the corresponding elastic energy density.

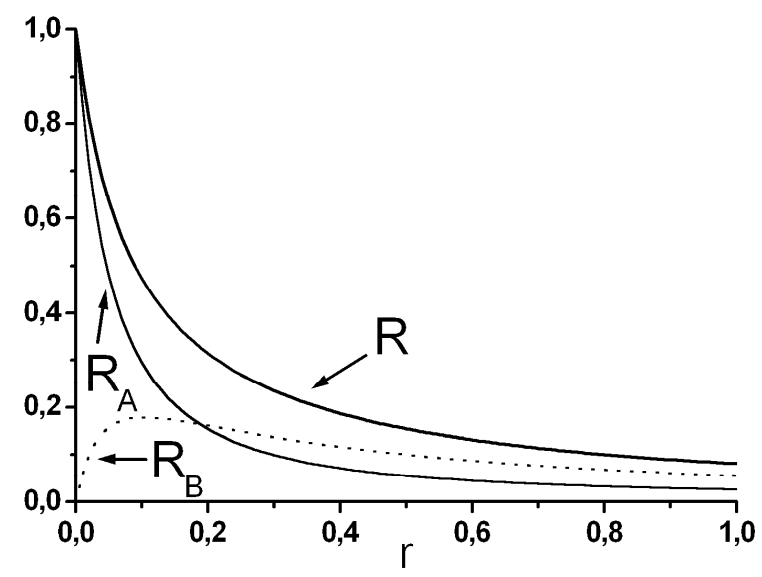

Finally it is very convenient to write the total energy change $\Delta F=\Delta F_{1}+\Delta F_{2}+\Delta F_{3}$ as a function of the volume of the 3D island $V=h \ell^{2}$, of the aspect ratio $r=h / \ell$, the number of underlying layers $\mathrm{z}$, and the film area $\mathrm{L}^{2}$ containing one island, using equations (28) to (31) $\Delta \mathrm{F}$ can be written

$$
\Delta F=-\Delta \mu\left(V+L^{2} z a\right)+\Phi_{\infty}\left[\left(L^{2}-\left(\frac{V}{r}\right)^{2 / 3}\right)\left(1-e^{-z}\right)+\left(\frac{V}{r}\right)^{2 / 3}\right]+4 \gamma_{A}^{\prime} V^{2 / 3} r^{1 / 3}+\mathrm{E}_{\delta}\left(V R(r)+z a L^{2}\right)
$$

where a is an atomic size and where for a box shaped crystal the relaxation factor only depends upon the aspect ratio $r=h / \ell$ and upon $\mathrm{z}$ for Stranski-Krastanov case.

Notice that if $2 D$ layers have to be formed A must wet $B$ and thus $\Phi_{\infty}$ must be negative (see (25)) Thus if $\Phi_{\infty}<0$ and $V>0, \Delta F$ given by (32) is the free energy change due to $S K$ condensation. The free energy change for single $2 D$ condensation (Frank van der Merwe growth) is thus simply obtained by taking $V=0$ with $\Phi_{\infty}<0$ in (32). On the contrary the free energy change due to $3 D$ growth onto a bare substrate (Volmer Weber growth) is obtained by taking $\Phi_{\infty}>0$ in (32) with $z=0$.

Globally in all cases condensation takes place for $\Delta \mathrm{F}<0$. To this can contribute the first two terms of (32): $\Delta \mu$ when positive and wetting $\Phi_{\infty}$ when negative. In the following, we will distinguish the growth of $2 \mathrm{D}$ layers and the subsequent growth of the $3 \mathrm{D}$ crystals.

\subsubsection{Equilibrium state}

The equilibrium state is found by minimisation of the total energy change $\Delta \mathrm{F}$, the zeros of the partial derivatives $\left.\frac{\partial \Delta F}{\partial z}\right|_{V, r} ;\left.\frac{\partial \Delta F}{\partial V}\right|_{z, r}$ and $\left.\frac{\partial \Delta F}{\partial r}\right|_{z, V}$ of (32) giving the equilibrium values of $\mathrm{z}, \mathrm{V}$ and $\mathrm{r}$ noted $\mathrm{z}^{*}, \mathrm{~V}^{*}$ and $\mathrm{r}^{*}$ respectively. In the following, supersaturation $\Delta \mu$ as well as 
$\theta=(\ell / L)^{2}$

the fraction of the film surface covered by 3D crystals are considered as constant parameters. According to the sign of the wetting energy $\Phi_{\infty}$ the three previous partial derivatives give the following relations given in table II where we distinguish $R^{3 D}(r)$ from $R^{S K}(r, z)$. We also put:

$r_{o}=\left|\Phi_{\infty}\right| / 2 \gamma_{A}$

We will show in section 3.2.3.2. that $r_{o}$ is the aspect ratio of a deposited crystal in absence of misfit.

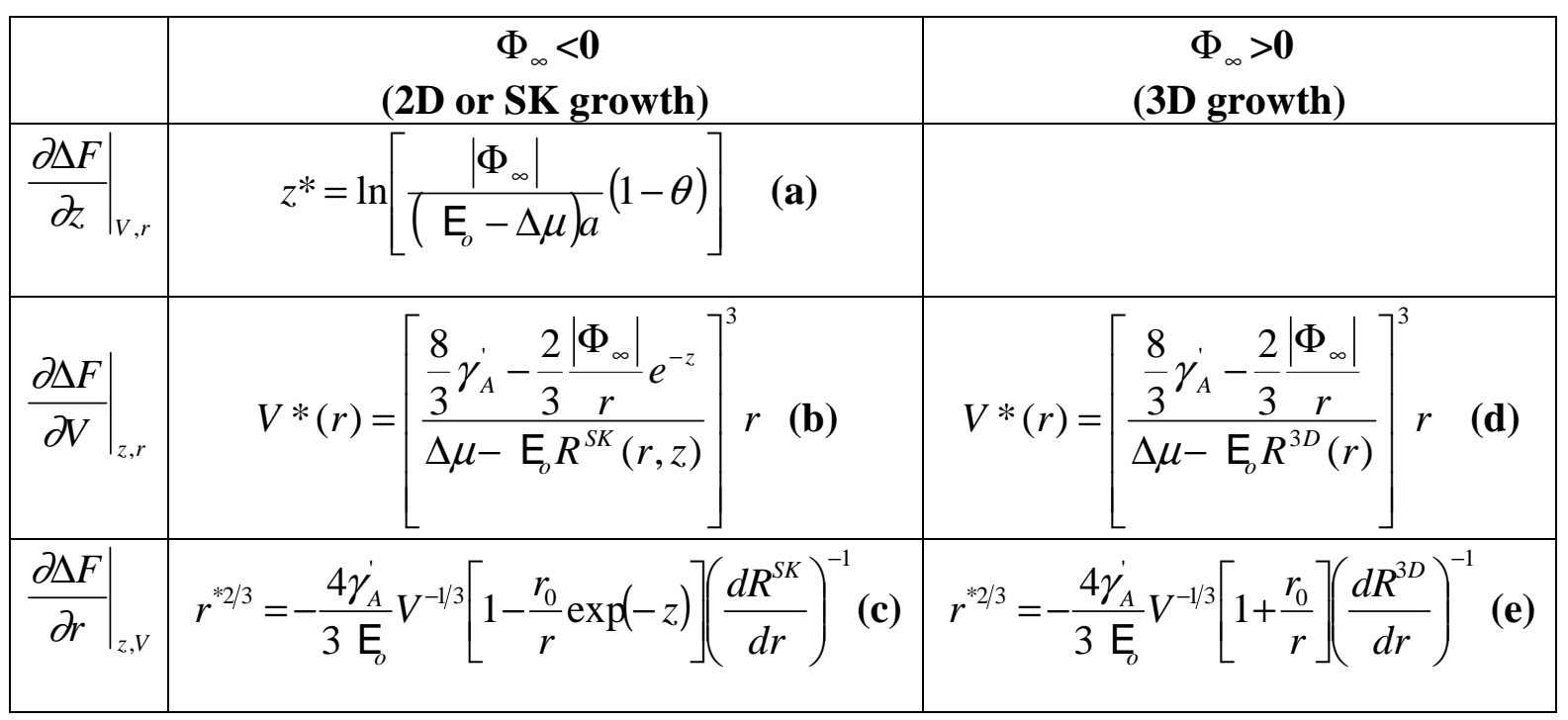

Table II: equilibrium values $z^{*}, V^{*}, r^{*}$ according to growth conditions

On the basis of these results, let us discuss the elasticity effects on the equilibrium state. In the three cases under study (2D, 3D, Stranski-Krastanov growth mode) we will consider growth conditions, then equilibrium properties and at last plastic-elastic interplay.

\subsubsection{Layer by layer growth}

* Here we are only concerned with Frank-van der Merwe growth that means $\Phi_{\infty}<0$ for having $2 \mathrm{D}$ condensation (see at the end of section 3.2.2.) with $\mathrm{V}=0$ and thus $\theta=0$ so that from formula (a) of table II there is

$$
z^{*}=\ln \left[\frac{\left|\Phi_{\infty}\right|}{\left(\mathrm{E}_{o}-\Delta \mu\right) a}\right]
$$

From (35), since $\mathrm{z}$ must be positive, the $\mathrm{z}$ layers can only exist for 
Thus for having 2D growth, the supersaturation cannot overpass the bulk elastic energy density stored in the strained layers. In absence of misfit $\left(\mathrm{m}_{\mathrm{o}}=0\right)$ the usual condition for $2 \mathrm{D}$ growth $\Delta \mu<0$ is recovered. $\mathrm{L}^{2} \rightarrow \infty$

For such layer by layer growth the free energy density of relation (32) reads with $V=0, r=0$,

$$
\Delta F / L^{2}=-\left(\Delta \mu-\mathrm{E}_{o}\right) z a-\left|\Phi_{\infty}\right|(1-\exp (-z))
$$
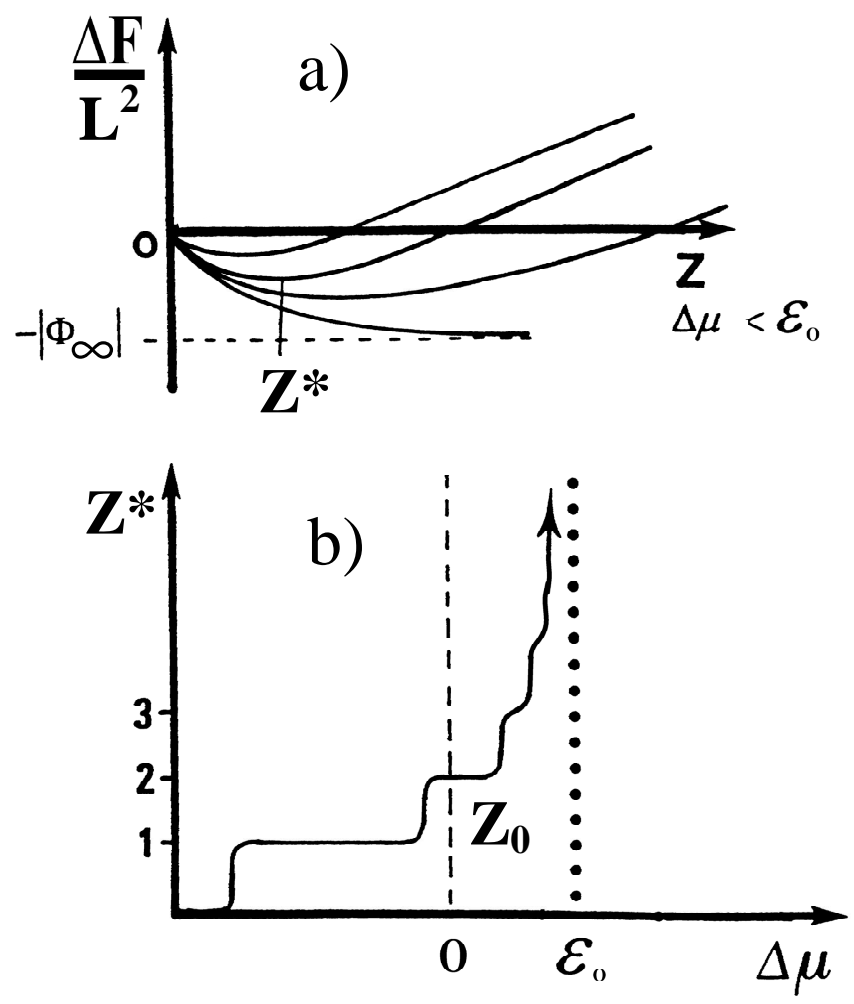

Figure 8:

a/ Free energy density change $\Delta F / L^{2}$ for layer growth as a function of the number of layers $z$ for different chemical potentials $\Delta \mu<\mathrm{E}_{0} . \Delta F / L^{2}$ only has minimum for $\Delta \mu<\mathrm{E}_{0}$.

b/ number of equilibrium layers $z^{*}$ versus the chemical potential $\Delta \mu$. For $\Delta \mu=\mathrm{E}_{o}, z^{*}$ tends towards infinity.

In figure 8a we plot the free energy density $\Delta \mathrm{F} / \mathrm{L}^{2}$ as a function of $\mathrm{z}$ for different chemical potentials $\Delta \mu . \Delta \mathrm{F} / \mathrm{L}^{2}$ shows minima, at $\mathrm{z}=\mathrm{z}^{*}$, for $\Delta \mu<\mathrm{E}_{o}$. In this case since $\left(\Delta F / L^{2}\right)_{z^{*}}<0$ $2 \mathrm{D}$ layers form spontaneously. Notice again that this spontaneous layer formation is precluded for $\Phi_{\infty}>0$.

* When relation (36) is fulfilled provided $\Phi_{\infty}<0$, each layer $\mathrm{z}$ is a $2 \mathrm{D}$ phase, built at a given undersaturation $\Delta \mu_{z}=\mathrm{E}_{o}-\left|\Phi_{\infty} / a\right| \exp (-z)$ obtained from (35). Up to saturation $\Delta \mu=0$ there builds up a finite number of layers $z_{0}$ (see fig $8 b$ ) which for a non covered film $\theta=0$ is given by:

$$
z_{o}=\ln \left[\frac{\left|\Phi_{\infty}\right|}{a \mathrm{E}_{o}}\right]
$$


This number only depends on the wetting over strain energy ratio $\left|\Phi_{\infty}\right| / a \mathrm{E}_{o}=\left|2 \gamma_{A}-\beta\right| /\left[Y m_{o}^{2}\right]$ (see (25) and (8)). This result is largely experimentally supported on very different pairs A/B: reversible multilayers adsorption measurements (see [76-79]). The result is remarkable so that it has to be illustrated with a striking picture: provided $2 \gamma_{A}-\beta<0$ a piece of material $A$ put in the vicinity of a crystal B exposing a (001) face, placed in a box of uniform temperature sublimates spontaneously on $B$ up to $z_{o}$ epitaxial layers. The only driving force to oppose to strain energy of the created A layers is due to $2 \gamma_{A}-\beta=\gamma_{A}+\gamma_{A B}-\gamma_{B}<0$. For Ge onto $\operatorname{Si}(111)$ one obtains $4<\mathrm{z}_{0}<5$ [56] in agreement with experimental data.

In fact (38) is only valid for the exponential behaviour of the long-range inter-layer potential we have chosen. More generally the equilibrium number of wetting layers depends upon the form of the long-range inter-layer potential. For instance if no such long range interactions is accounted for and only short range interactions are supposed to act as in first neighbours model, there is a cut-off for $\mathrm{z}=1$ that means $\Phi(z=1)=\Phi_{\infty}$ but $\Phi(z>1)=0$. Then it results only one equilibrium wetting layer $\mathrm{z}=1$ (see appendix $\mathrm{E}$ ). This is however an extreme prevision since to such short-range forces adds some long-range contribution.

In the case of long range contribution and of finite misfit it is seen that when $\Delta \mu=\mathrm{E}_{0}$ the number of equilibrium layers $z^{*}$ given by (35) becomes infinite (see fig. 7). In the case of a vanishing misfit $\mathrm{m}_{0} \rightarrow 0$ the number of layers $\mathrm{z}_{\mathrm{SK}}$ given by (35) tends towards infinity too, even at $\Delta \mu=0$. Obviously latter layer-by-layer growth is quite normal but in the $m_{0} \neq 0$ case the elastic energy stored increases with the number of layers $\mathrm{z}$ so that the system has to relax either by plastic deformation or islanding.

* Let us first consider relaxation by interfacial dislocation insertion. The critical number of layers beyond which dislocations may appear can be obtained following a simple treatment of Matthews [80]. From a thermodynamical point of view the number of interfacial dislocations may pass from $\mathrm{N}$ to $\mathrm{N}+1$ when the total elastic energy change due to the introduction of the $(\mathrm{N}+1)^{\text {th }}$ dislocation is negative. It is easy to show (see end of appendix $F$ with $K=1$ and $b=a$ ) that the critical number of layers $z_{\text {disl }}$ beyond which dislocations may thermodynamically insert roughly is the solution of the following equation

$$
z_{\text {disl }} \approx \frac{1}{4 \pi} \frac{1+\ln z \text { disl }}{m_{o}}
$$

Conversely this relation tells that for $\mathrm{z}>\mathrm{z}_{\mathrm{dis}}$, the misfit $\mathrm{m}_{\mathrm{o}}$ or the mean strain in the layers decreases roughly as the inverse of the number of deposited layers and thus reduces the elastic energy and therefore the equilibrium number of layers given by (35) for fixed $\Delta \mu$. Obviously owing to kinetic reasons dislocations may only enter for greater thicknesses.

* The case of elastic relaxation by islanding concerns the Stranski-Krastanov transition and will be treated in section 2.2.3.3. At this stage we will only notice that beyond some new critical number of layers we will call $\mathrm{z}_{\mathrm{SK}}$, 3D islanding may occur. Thus according to the relative values of $z_{\text {disl }}$ (varying as $1 / \mathrm{m}_{0}$ see (39) and $\mathrm{z}_{\mathrm{SK}}$ (varying as $\ln \left(1 / m_{o}^{2}\right.$ ) see formula (a) table II) relaxation takes place, at thermodynamical equilibrium, either by islanding or dislocation entrance. Furthermore since activation energies for dislocation entrance and 3D islanding behave as $m_{o}^{-2}$ and $m_{o}^{-4}$ respectively [81] there is really also a kinetics competition between these two modes of relaxation. 
As a partial conclusion at equilibrium, elasticity modifies the chemical potential of each layer and fixes the number of layers at $\Delta \mu=0$ (see (38)). However since elastic energy diverges with $z$, beyond some critical number of layers elastic relaxation by dislocation entrance or islanding occurs.

\subsubsection{3D growth on a bare substrate $\left(\mathrm{z}=0, \Phi_{\infty}>0\right)$ : Volmer-Weber case}

* In this case the relation (d) in table II gives the equilibrium volume $\mathrm{V}^{*}$ of the island. This relation says:

(1) The value of the chemical potential $\Delta \mu$ selects the volume of the crystal. More precisely the greater $\Delta \mu$, the smaller the size of the equilibrium crystal. Typically this effect is the usual Gibbs Thomson behaviour for first order phase transitions.

(2) However here $3 \mathrm{D}$ crystals can only exist when $\mathrm{V}^{*}(\mathrm{r})>0$, that means when

$\Delta \mu>\mathrm{E}_{0} R^{3 D}(r)$.

If the elastic relaxation effect is neglected, $R^{3 D}(r)=1$, the 3D crystal can only exist at $\Delta \mu>\mathrm{E}_{0}[87]$.

* Relation (e) in table II describing the equilibrium shape ratio of the 3D crystal is more interesting. It can be rewritten as a parametric system in $\mathrm{r}$ with $\mathrm{h}^{*}$ and $\ell^{*}$ the equilibrium height and length of the 3D crystal $[65,79,87]$

$$
\left\{\begin{array}{c}
h^{*}=-\frac{4 \gamma^{\prime}}{3 \mathrm{E}_{o}}\left[1-\frac{r_{0}}{r}\right]\left(\frac{d R^{3 D}}{d r}\right)^{-1} \\
\ell^{*}=h^{*} / r
\end{array}\right.
$$

Where $r_{o}$ is given by (34)(25). For $m_{0}=0$ the system gives the usual Wulf-Kaishew theorem [82-86]

$$
h * / \ell^{*}=r_{0}=\frac{2 \gamma_{A}-\beta}{2 \gamma_{A}^{\prime}}
$$

whose principal meaning is that the aspect ratio $r=r_{0}$ is size independent (for a discussion see $[65,87])$

In the presence of misfit, $\mathrm{E}_{o} \neq 0$, the system (41) only has a solution for $\mathrm{r}>\mathrm{r}_{\mathrm{o}}$. This means that epitaxially box shaped strained crystals must have greater aspect ratio than the strainfree crystal. More precisely, (1) epitaxial strain acts against wetting (adhesion) so that globally it leads to a thickening of the equilibrium shape; (2) owing to strain this equilibrium shape becomes size dependent.

Obviously relation (41) can only be used practically when the relaxation factor for Volmer Weber growth has been calculated for the shape family $\mathrm{r}$ under study. For a box shaped crystal an analytical form of $R^{3 D}(r)$ has been calculated [65,87]. In figure 7 we plot from [65] 
the relaxation factor of a box shaped crystal when deposit and substrate have the same elastic properties. It is seen that when $\mathrm{r}=0$, a uniform coherent film is not relaxed $\left(R^{3 D}(0)=1\right)$. However as the deposit becomes a rectangular box (finite $r$ ) it relaxes from its borders so that $R^{3 D}(r)<1$ and therefore it stresses the underlying substrate (for a more complete discussion about relaxation factors for various shapes see [88]). Let us note that for $5<1 / r<50 R^{3 D}(r)$ can be roughly fitted by $R^{3 D}(r) \approx 0.14 r^{-1 / 2}$.

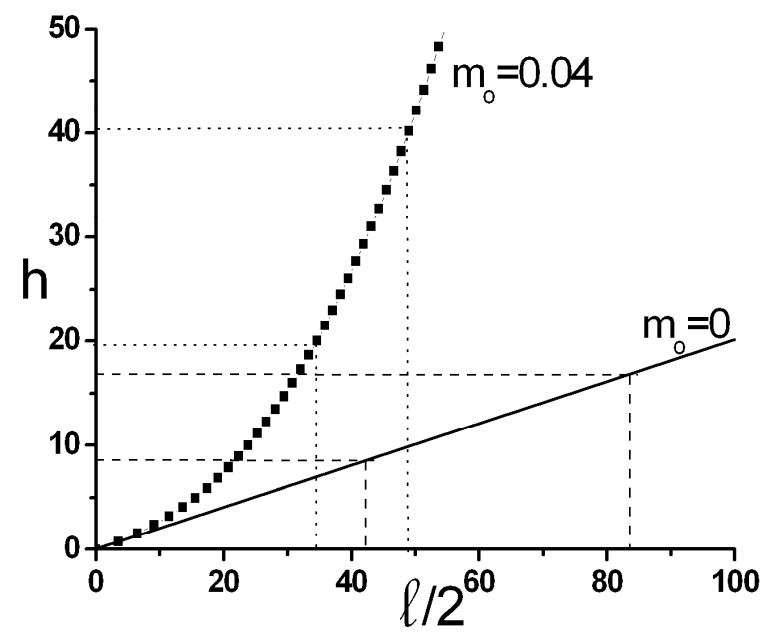

Figure 9: Half equilibrium shape of a $3 D$ box shaped crystal (discontinuous lines) $h$ : height, $\ell / 2$ : half basis in atomic units. Continuous curves are the corner trajectories calculated for $r_{o}=0.1, \mathrm{~m}_{\mathrm{o}}=0$ (straight line) or $\mathrm{m}_{\mathrm{o}}=4 \%$ (squares). The epitaxial misfit leads to a thickening of the ES. Furthermore similarity is lost.

Calculating then $\mathrm{dR} / \mathrm{dr}$ from figure 7 we can plot from (41) $h_{e q}=h_{e q}\left(\ell_{e q}\right)$ for a given wetting factor $r_{o}$ and $\gamma_{/ /} / E$ value, a so-called elasto-capillary length where $E$ is Young's modulus in an isotropic surface of surface energy $\gamma$. This length scales $10 \gamma / Y \approx 10^{-8} \mathrm{~cm}$ that means with the size of an atom as mentioned by F.C.Frank [45]. We will use it in the following discussion. The result of the resolution of (41) is shown in figure 9 for $r_{0}=0.1$, for $\mathrm{m}_{\mathrm{o}}=0$ and $\mathrm{m}_{\mathrm{o}}=4 \%$. Each curve figures the trajectory of the edge of the half equilibrium shape (ES) with size.

For $\mathrm{m}_{\mathrm{o}}=0$ the usual Wulf-Kaishew theorem still holds and the equilibrium aspect ratio $\mathrm{r}=\mathrm{r}_{\mathrm{o}}$ is size independent (ES are obtained from the straight line on figure 8) or more generally they have similarity. For $m_{0} \neq 0$ the ES ratio increases with size so that similarity is no more preserved. A more complete discussion about wetting and relative stiffness can be found in $[65,87]$.

Thus as a partial conclusion, in presence of elasticity $3 D$ growth takes place when supersaturation overpasses the bulk elastic energy (see (40)) and the ES of the growing crystal is modified in such a manner the greater the misfit, the higher the equilibrium shape. For other polyhedral shapes the relaxation factor can be calculated by numerical methods [6165]. Elastic effects on truncated pyramids [60,62,79,87], 2D cylinders [63] or 3D spheres [64] have been considered. Nevertheless the main effect is the same (excepted when the island shape has been fixed as in [64]): a thickening of the ES but furthermore the various facets extension changes with size, some facets decreasing, other increasing [65,87].

* Obviously this scenario cannot be valid whatever the size. Indeed as for 2D film (see section 3.2.3.1.) the growing crystal accumulates elastic energy, in spite of elastic relaxation which can become prohibitive so that plastic relaxation occurs. The treatment is similar as in section 3.2.3.1. One finds (see appendix F) that a first dislocation orthogonal pair may 
thermodynamically enter in the island interface as soon as a critical height $\mathrm{h}_{\mathrm{C}}$ is reached. It is obtained as a solution of:

$$
h_{c} \approx \frac{1}{4 \pi} \frac{1+\ln h_{c}}{\left(m_{o}-2 / \ell\right) R^{3 D}(r)}
$$

Each supplementary dislocation entrance abruptly drops the strain from $m_{0}$ to $m^{\prime}=m_{0}-N b / \ell_{N}$ (see appendix $\mathrm{F}$ ) where $\mathrm{b}$ is the Burgers vector modulus of the interfacial dislocation and $\ell_{N}$ the lateral size of the crystal in which enters the $\mathrm{N}^{\text {th }}$ dislocation. Then since we have seen that the equilibrium shape is strain dependent, each dislocation entrance abruptly modifies the equilibrium shape [65,87,88,89] (see figure 10 and its caption). More precisely since the smaller the misfit, the flatter the crystal, the main effect of dislocation entrance thus is a back flattening of the equilibrium shape. Such a quick variation of the equilibrium shape at each dislocation entrance has been experimentally shown by F.Legoues et al. [90,91]. For shapes more complex than box shaped crystals there is a jerky modification of the various facets extension $[65,88]$.

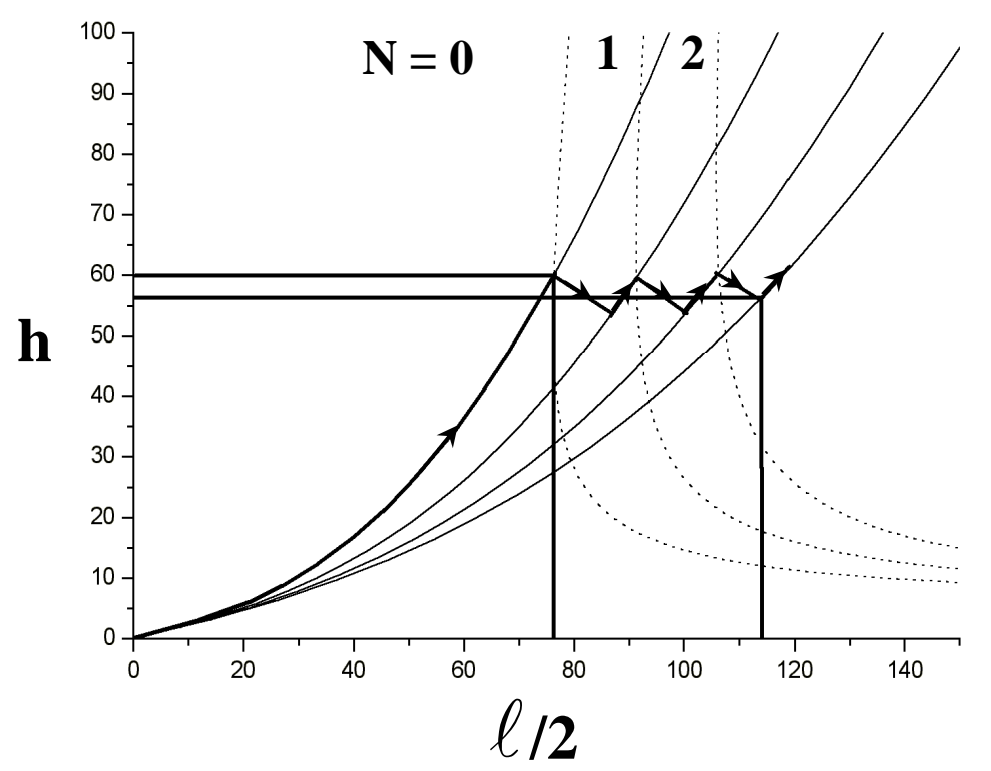

Figure 10: Effect of dislocation entrance on the ES. The box shaped crystal accumulates strain energy when growing along the $h(\ell / 2)$ curve with an arrow until it reaches some critical size (here for $r_{o}=0.1$ and $m_{0}=4 \%, h_{0}=61, \ell_{0}=155$ ) where a first dislocation may thermodynamically enter (dotted curves represent the thermodynamic criterion for the first $(N=0)$ dislocation entrance (eq. iii appendix $F$ ). If the dislocation effectively enters for this size, according to equation (ii) appendix $F$ the misfit passes from $m_{o}$ to $m_{o}-1 / \ell_{o}$. The ES of the growing crystal thus must follow a new ES trajectory re-calculated from (41) but with the new misfit.If it is assumed that the island changes its shape at constant number of atoms, the crystal abruptly flattens. The same thing occurs for the second $(N=1)$, third $(N=2)$, and fourth $(N=3)$ dislocation entrance where in the case under study the misfit passes respectively from $3.4 \%$ to $2.9 \%$ then $2.6 \%$. 


\subsubsection{2D relayed by $3 D$ growth $\left(\Phi_{\infty}<0\right)$ : Stranski-Krastanov case}

* From the condition of existence of $\mathrm{z}^{*}>0$ and $\mathrm{V}^{*}>0$ obtained from equations (b) and (c) in table II Stranski Krastanov growth can only occur, in near equilibrium conditions, when the following relation is fulfilled:

$$
\mathrm{E}_{o} R^{S K}(r, z)<\Delta \mu<\mathrm{E}_{0}
$$

that means in a finite domain of chemical potential $\Delta \mu$ where now $R^{S K}(r, z)<1$ describes the elastic relaxation of a $3 \mathrm{D}$ crystal sitting on $\mathrm{z}$ pseudomorphic layers covering the lattice mismatched substrate. In absence of elastic relaxation $\left(R^{S K}(r, z)=1\right)$ according to (44) there is no more place for Stranski Krastanov mode in near equilibrium conditions. Elastic relaxation thus is a prerequisite for the simultaneous existence of $2 D$ layers and $3 D$ crystals.

Figure 11: Number of equilibrium layers $z^{*}$ versus the chemical potential $\Delta \mu$ in case of $S K$ growth. For $\mathrm{m}_{\mathrm{o}} \neq 0,3 D$ islands may appear as soon as $\Delta \mu>\mathrm{E}_{0} R$. corresponding to $z>z_{S K}$.

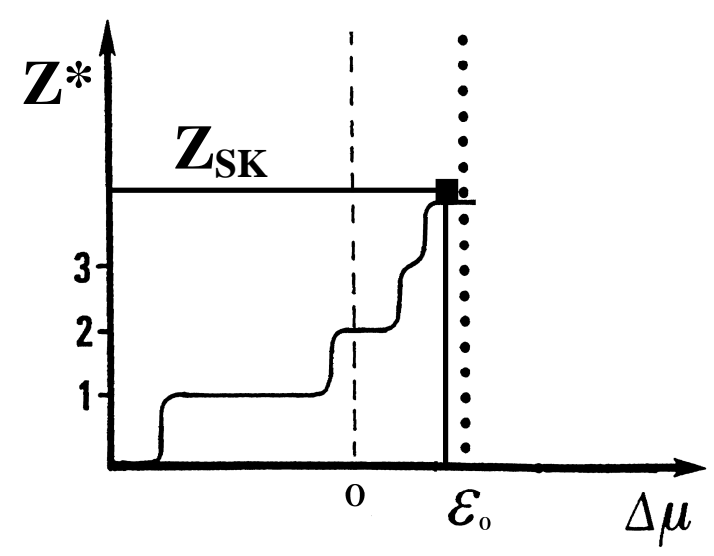

In figure 11 we schematically plot the number of 2D layers as a function of the chemical potential $\Delta \mu$. To each layer formation $z^{*}$ corresponds a constant value of $\Delta \mu$ given by $\Delta \mu\left(z^{*}\right)=\mathrm{E}_{o}-e^{-z^{*}} \Phi_{\infty}(1-\theta)$. For $\mathrm{m}_{0}=0$ the number of steps becomes infinite at saturation $\Delta \mu=\mathrm{E}_{\mathrm{o}}=0$ (fig.7b). For $\mathrm{m}_{\mathrm{o}} \neq 0$ 3D islands may appear as soon as $\Delta \mu>\mathrm{E}_{0} R^{S k}(r, z)$. Therefore in figure 11 for increasing $\Delta \mu$ there is a cut-off at $\Delta \mu=\mathrm{E}_{0} R^{S K}(r, z)$ where 3D crystals may appear on the $\mathrm{z}_{\mathrm{SK}}$ underlying layers. Beyond $\Delta \mu=\mathrm{E}_{0}$ the representation does not make sense, exactly as when $\mathrm{m}_{0}=0$ for $\Delta \mu>\mathrm{E}_{0}=0$ (see fig. $7 \mathrm{~b}$ ). Let us remark that because of the limitation $\Delta \mu \leq \mathrm{E}_{o}$ the smallest volume a $3 \mathrm{D}$ crystal can reach is obtained by injecting $\Delta \mu=\mathrm{E}_{0}$ in the expression of $\mathrm{V}^{*}$ in table II. For a given aspect ratio $\mathrm{r}$ and for $\mathrm{z} \rightarrow \infty$ this minimum volume reads:

$$
V_{\min } *(r)=\left[\frac{\frac{8}{3} \gamma_{A}^{\prime}}{\mathrm{E}_{o}\left(1-R^{S K}(r, z)\right)}\right]^{3} r
$$


Here again we see that elastic relaxation $R^{S K}(r, z)<1$ is a prerequisite for Stranski Krastanov mode. Indeed for $R^{S K}(r, z)=1$, relation (45) says that the 3D crystal must have an infinite volume! However, because of the fact that the usual activation barrier for 3D nucleation is proportional to the one third of the total surface energy of the nucleus [6,92], nucleation of such large crystals should be difficult. But since SK mode exists the true growth mechanism must minimize this activation energy. We will come back to this point in section 3.2.3.5.

In fact, at equilibrium the chemical potential $\Delta \mu$ must be the same for the $2 \mathrm{D}$ layers and the $3 \mathrm{D}$ crystal. Combining thus $\mathrm{z}^{*}$ and $\mathrm{V}^{*}$ expressions (a) and (b) of table II, $\Delta \mu$-independent equilibrium values $\mathrm{z}^{*}$ and $\mathrm{V}^{*}$ can be easily obtained. Thus for each value of $z^{*}$ there exists an aspect ratio $r$ which minimises the crystal volume $V^{*}(r)$ which can co-exist on $z^{*}$ layers.

At this stage we can summarise the conditions of Stranski-Krastanov transition onto a dislocation free 2D film:

$$
\left\{\begin{array}{c}
\mathrm{E}_{o} R^{S K}(r)<\Delta \mu<\varepsilon_{o} \\
z_{o} \leq z(r)<z_{d i s l} \\
V(r)>V_{\min }^{*} \propto m_{o}^{-6}
\end{array}\right.
$$

* Concerning the equilibrium shape the main differences between Volmer-Weber and Stranski-Krastanov cases are:

(1) In the expression of the equilibrium shape ratio $r^{*}$ the factor $1-r_{o} / r$ which appear in formula (41) in case of Volmer Weber (VW) growth has to be be replaced by $1+r_{o} e^{-z} / r$ in case of Stranski-Krastanov growth. This originates in the now negative wetting energy $\Phi_{\infty}$ (necessary to build z pseudomorphous layers) which decreases with the film thickness z. Owing to this difference, positive height $h^{*}{ }_{V W}$ can only exist for $\mathrm{r}>\mathrm{r}_{\mathrm{o}}$ in the VW case whereas crystal flatter than $\mathrm{r}=\mathrm{r}_{\mathrm{O}}$ can exist in the SK case.

(2) The relaxation factors $R^{3 D}(r)$ and $R^{S K}(r, z)$ appearing in table II are not the same.

As still mentioned relaxation factor $R^{S K}(r, z)$ for $3 \mathrm{D}$ crystals onto z pseudomorphic layers have not been calculated. Nevertheless if the number of underlying layers is great enough, it can be considered that the 3D crystal grows onto a homogeneously strained semi-infinite substrate of A. In this case it must be $R^{S K}(r, z)=R^{3 D}(r)$ and thus the equilibrium shape can be obtained (for $\mathrm{z} \rightarrow \infty$ ) from the following parametric equations

$$
\left\{\begin{array}{c}
h^{*}=-\frac{4 \gamma_{A}^{\prime}}{3 \mathrm{E}_{\mathrm{o}}}\left(\frac{d R^{3 D}}{d r}\right)^{-1} \\
\ell^{*}=h^{* / r}
\end{array}\right.
$$

Thus since for box shaped crystals an analytical form of $R^{3 D}(r)$ has been found [87] (see also 2.2.3.2.) it is possible to plot the equilibrium shape of the SK 3D crystal. 


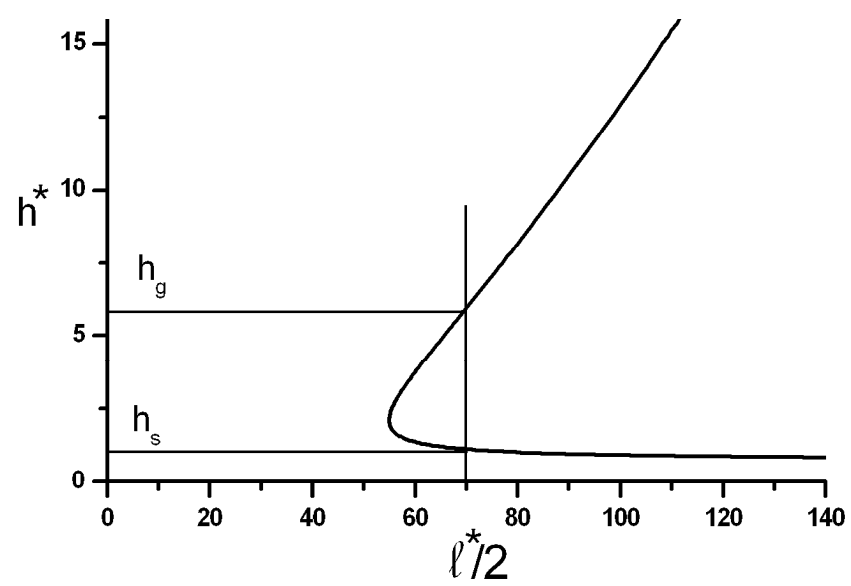

Figure 12a: Corner trajectory of the ES $h=f(\ell / 2)$ for Stranski Krastanov case $\left(r_{o}=0\right)$ calculated for $\mathrm{m}_{\mathrm{o}}=4 \%$.

Figure 12b: Free energy change (32) as a function of the aspect ratio of the growing crystal for $V<V_{\text {min }}^{*}(r)$ where there is no minimum (excepted in $r=0$ ) and for $V>V_{\text {min }}^{*}(r)$ where the minimum defines the equilibrium aspect ratio, the maximum a labile state to overpass.

\section{$x$}

We plot on figure 12a such an ES, the graph $\mathrm{h}^{*}\left(\ell^{*}\right)$ having now two branches which correspond to the extrema of $\Delta \mathrm{F}(\mathrm{r})$ (32) and define two equilibrium aspect ratios $\mathrm{r}_{\mathrm{g}}$ (great) and $r_{s}$ (small) or two equilibrium heights $h_{g}$ and $h_{s}$ provided the volume $\mathrm{V}$ is greater than $V_{\text {min }}^{*}$ (45). The lower branch (flat crystals with $r=r_{\text {min }}$ ) is the locus of the maximum of $\Delta F(r)$ (crest) (see figure12b) and thus describes a labile equilibrium whereas the upper branch (thick crystal with $r=r_{g}$ ) is the locus of the minimum of $\Delta F(r)$ (valley) and thus describes the stable equilibrium analogous to what we depicted for Volmer Weber growth (see section 3.2.3.2.). Obviously (see figure 12b) the transformation at constant volume from labile equilibrium (crest) to stable equilibrium (valley) is spontaneous [56,65].

From a theoretical point of view we have seen that since for SK growth $\mathrm{z}^{*}$ depends upon the 3D islands coverage $\theta=(\ell / L)^{2}$ (see $\mathrm{z}^{*}$ for SK growth in table II) the greater the volume of the $3 \mathrm{D}$ crystal the smaller the number of underlying layers at equilibrium. Most experiments agree with this description since they clearly show that Stranski-Krastanov transition can occur at a constant number of deposited atoms and that 3D growth occurs at the expense of the 2D layers. In other words owing to the elastic relaxation of $3 D$ crystals, some of the upper layers of a metastable $2 D$ strained-film (thickness $z$ ') can transform into stable $3 D$ islands leading to the Stranski-Krastanov situation where the remaining $z^{*}$ layers support these $3 D$ 
islands. In a paper we have shown how owing to strain relaxation sufficiently large 2D islands of simple height double their height and start the SK transition [56].

* Obviously, once more the stored elastic energy increases with the number of deposited atoms so that beyond some critical size dislocations may enter the system. If $\mathrm{z}_{\mathrm{Disl}}<\mathrm{z}_{\mathrm{SK}}$ the dislocations may enter in the film before Stranski-Krastanov transition takes place. Thus since dislocation entrance decreases the misfit, Stranski-Krastanov transition can no more occur even for further growth. Since on one hand the greater the wetting the greater $z_{S K}$ whereas on the other hand the smaller the relative rigidity $\mathrm{K}$ (see appendix $\mathrm{F}$ ) the smaller $\mathrm{z}_{\text {Disl }}$, for weak wetting and sufficiently soft substrate there can also be $z_{D i s l}>z_{S K}$. Obviously as for VolmerWeber growth kinetics may modify this condition. When $z_{\text {Disl }}>z_{S K}$, naturally arises the question of the localisation of the dislocations. Are they at the bottom of the layers or at the bottom of the deposited crystals? The question remains open and the answer must depend, at equilibrium, upon the shape and the density of islands (in section 4. we will say some words on interacting crystals) as well as on the relative substrate to deposit rigidity since dislocations always go towards the softer material.

\subsubsection{Comments on surface stress effects}

Until now we have not considered surface stress effects in the just foregoing discussion. When such effects are included in the formulation of $\Delta \mathrm{F}$ of (32) by means of the surface work during accommodation and relaxation, elastic relaxation and equilibrium shape calculations can no longer be explicitly solved (see for example [93]). Nevertheless surface stress has several predictable main effects

(1) For $2 \mathrm{D}$ growth, since the surface stress modifies the wetting factor from $\Phi_{\infty}$ to $\Phi_{\infty}^{m_{o}}$ (see (25) and (27)) it modifies the number of equilibrium layers. Surface stress also plays a role on the critical number of layers beyond which dislocations may thermodynamically appear. However since in this case the film relaxes by dislocation entrance the interfacial stress $s_{A B}{ }^{\dagger \dagger}$ also works [93]. It is thus easy to show (see appendix $G$ ) that for positive natural misfit $\mathrm{m}_{\mathrm{o}}$ a positive $\Delta s^{\infty}=s_{A}+s_{B}-s_{A B}$ value (where $\mathrm{s}_{\mathrm{A}}$ and $\mathrm{s}_{\mathrm{B}}$ are the surface stresses of deposited crystal A and substrate B respectively) lowers the critical thickness $h_{c}$. On the contrary a negative $\Delta s^{\infty}$ increases the critical thickness beyond which dislocations may appear. For Ge/Si(100) $\left(\mathrm{m}_{\mathrm{o}}=-4 \%\right)$ Floro et al. [94] give $\Delta s^{\infty}=2.3 \mathrm{Jm}^{-2}$. In this case $\mathrm{m}_{\mathrm{o}} \Delta s^{\infty}<0$ so that the critical thickness $h_{c}$ is decreased by surface and interface stresses from 5.4 monolayers to 2.6 monolayers (see figure 13 where we plot the equilibrium strain versus the deposit height $\mathrm{h}$ for $\Delta s^{\infty}=0$ and $\Delta s^{\infty}= \pm 2.3 \mathrm{Jm}^{-2}$ ). Nevertheless such a reversible critical thickness dependence with misfit and surface stress is difficult to put in evidence since dislocation formation is an activated process so that the kinetic critical thickness beyond which dislocations effectively occur generally is greater than the predicted thermodynamical value.

(2) For 3D growth let us recall that taking into account surface stress work against surface deformation is equivalent to taking into account the first order development with strain of the surface energy (see comments just after (27)). Thus the gamma plot of the strain free equilibrium shape is modified by strain. Nevertheless since in most cases the surface energy

\footnotetext{
${ }^{\dagger}$ In fact for semi coherent interface composed of a grid of dislocations one needs to distinguish two interfacial stresses. Indeed the usual interfacial stress is defined as the work done to deform both facing phases by the same amount, whereas in the presence of dislocations changing the in plane parameter of the substrate needs dislocation introduction (See [95])
} 
change versus strain remains weak (of the order of $(s-\gamma) \varepsilon$ see (27)) surface stress is not really effective when elastic relaxation operates (see for example [96]). Nevertheless things can be different when new surface phases can be stabilized by external stress. Indeed in this case stress-induced changes of surface structures (first order transition) may lead to a discontinuous change of surface energy and surface stress and thus of the gamma plot. In this case new cusps could appear on the gamma plot and thus new stress-stabilized faces may appear on the equilibrium shape. We believe [89] that this happens for the well defined $\{105\}$ facets of the so called "huts" appearing during the first stages of the growth of $\mathrm{Ge} / \mathrm{Si}(100)$ $[97,98]$.

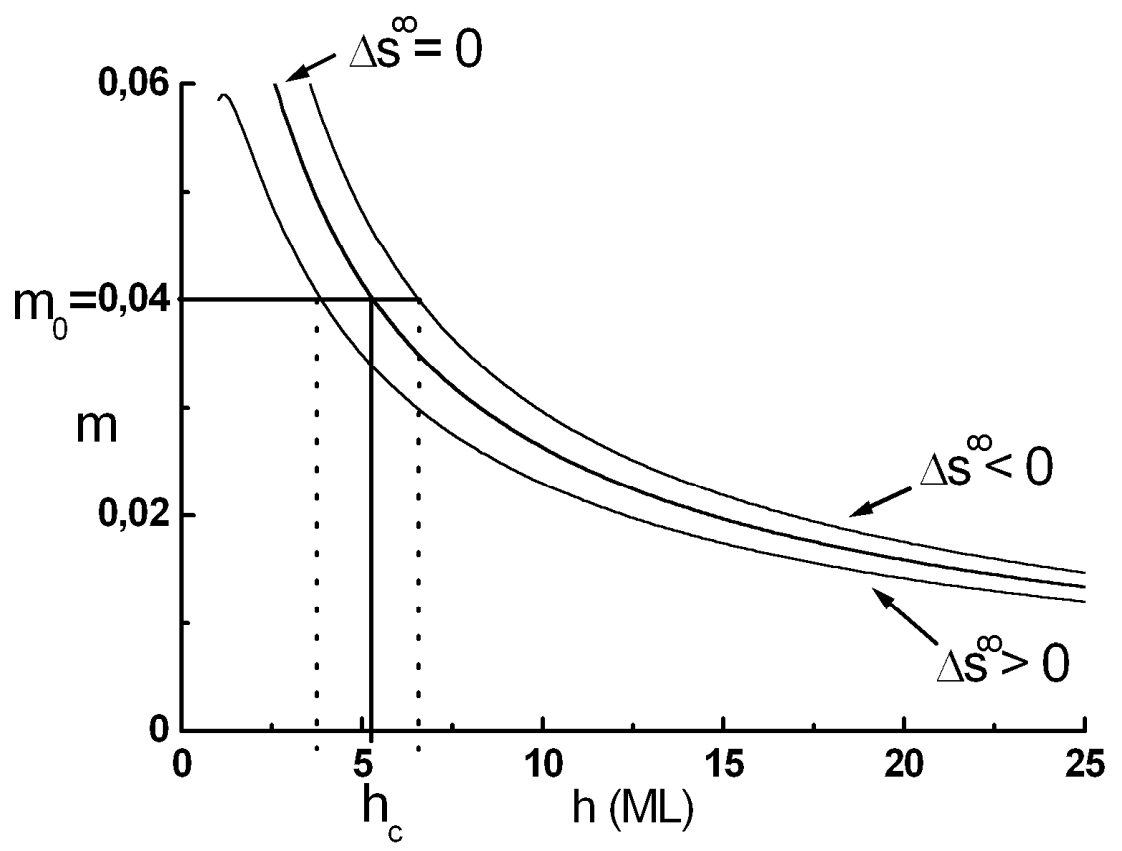

Figure 13: Residual misfit $m$ 'versus the film thickness $h$ calculated for $m_{o}=-4 \%$ and $\Delta s^{\infty}=0 \pm 2.3 \mathrm{Jm}^{-2}$ (see appendix $G$ ). The growing film remains pseudomorphous to its substrate up to $h=h_{c}$. Beyond this critical thickness $h_{c}$ the misfit is partially accommodated by dislocation entrance and the residual elastic misfit decreases with the thickness $h>h_{c}$ of the film. For $\Delta s^{\infty}=0$ and $m_{o}=-4 \%, h_{c} \approx 5.4 \mathrm{ML}$. A positive surface stress change $\Delta s^{\infty}=2.3 \mathrm{Jm}^{-2}$ decreases $h_{c}$ to $2.6 \mathrm{ML}$ whereas negative surface stress change $\Delta s^{\infty}=-2.3 \mathrm{Jm}^{-2}$ increase $h_{c}$ to $h_{c} \approx 7.5 \mathrm{ML}$. The case Ge/Si (100) corresponds to $m_{o}=-4 \%, \Delta s^{\infty}=2.3 \mathrm{Jm}^{-2}$ [94] and thus $h_{c} \approx 2.6$ $M L$.

It was found in Molecular Beam Epitaxial growth that some foreign adsorption plays a role on the equilibrium state. In the case of $2 \mathrm{D}$ growth, such additives can modify the number of equilibrium layers $\mathrm{z}[99,100]$, whereas for 3D growth they can modify the equilibrium shape of the growing crystal [101]. Such additives are known to lower the surface energy [5] without bulk incorporation and thus were called surfactants by the semiconductor community. Nevertheless since surface energy $\gamma$ is lowered by adsorption, and since Shuttleworth relation (10) connects surface energy to surface stress, consequently surface stress changes with adsorption. More precisely since there is $\partial \gamma / \partial \mu=-\Gamma$ where $\mu$ is the chemical potential of the adsorbed species and $\Gamma$ the adsorption density (generally positive see section 1.4.) there is $\partial s / \partial \mu=-\Gamma-\partial \Gamma / \partial \varepsilon$. The adsorption may thus reduce or enhance the surface stress. Such 
surface stress changes with adsorption have been reported (for example see the important review paper by Ibach [102] its erratum [103] and [104]). Therefore surfactants not only play on the surface energy but also change the mechanical state of the crystal.

\section{ELASTIC EFFECTS ON ATOMISTIC MECHANISMS}

We recall some well-known facts for non-stressed crystals.

In the case of a flat $(F)$ defect-free surface ${ }^{*}$ the rate of growth is determined by the frequency of formation of $2 \mathrm{D}$ or $3 \mathrm{D}$ nuclei. The nuclei formation requires overpassing an activation barrier. Supersaturation decreasing this barrier, a critical supersaturation has to be overcome for the growth to take place. We will see in section 3.1 how elasticity can influence the $2 \mathrm{D}$ and $3 \mathrm{D}$ nucleation process. For a stepped face $(\mathrm{S})$ with an orientation along an inward cusped valley, the growth may occur by step flow mechanism as first depicted by Burton Cabrera and Frank (BCF) [105]. In their seminal paper the authors consider kinetics of growth of a vicinal surface as a balance between adatom deposition, adatom diffusion and adatom attachment to steps (for a review see $[92,106]$ ). If the sticking probabilities of adatoms from the upper terrace and lower terrace are equal, all the steps have the same velocity. Such growth mode is known as step flow and occurs at small supersaturation. In fact at higher supersaturation there is a transition from this step flow mode to 2D nucleation mechanism in between the steps. When no reevaporation takes place, or the diffusion length onto the terrace is much greater than the distance between steps, 2D nucleation on the terrace is more favourable. When the nucleus only contains one atom (very high supersaturation, see appendix $\mathrm{H}$ ) this transition occurs when $(\mathrm{D} / \mathrm{F})^{1 / 6} \approx \mathrm{L}$ where $\mathrm{L}$ is the step to step distance, $\mathrm{D}$ the surface diffusion constant and F the impinging flux [106,107]. Such transition from step flow to $2 \mathrm{D}$ nucleation is easy to detect experimentally. Indeed in presence of $2 \mathrm{D}$ nucleation birth and spread of 2D islands give oscillations of the RHEED intensity at each completion of layer whereas in step flow mode there are no oscillations $[108,109]$. In the case of a flat $(F)$ face but with screw dislocations 2D nucleation is shunted at low supersaturation [105] and steps with high Burgers modulus $\mathrm{b} \perp$ propagate laterally each anchored at the dislocation so that equidistant steps are winded as helices. This gives very flat growth pyramids around each non-cooperating screw dislocation. Their slope is proportional to $1 / \Delta \mu$ [105].

Obviously elasticity may influence some of these elementary processes involved in step flow. In the framework of these lectures the various elastic effects on adatom detachment rate and surface diffusion will be discussed separately (section 3.2. and 3.3.). In each case we will not give the details of the calculations but only try to capture the essential physics for simple cubic material $\left(\gamma_{A}=\gamma_{A}^{\prime}\right)$.

\subsection{Nucleation barrier}

* On a flat perfect surface (F), crystal growth takes place after 2D or 3D nucleation. On average, clusters smaller than the so-called critical nucleus spontaneously disappears whereas clusters larger than the critical nucleus spontaneously grow. The critical nucleus size depends on supersaturation $\Delta \mu$ and its formation requires overpassing an activation barrier. In principle the activation barrier $\Delta \mathrm{F}^{*}$ is obtained by injecting the equilibrium nucleus parameters $h^{*}, \ell^{*}$ or $\mathrm{V}^{*} \mathrm{r}^{*}$ (table II) in the free energy change $\Delta \mathrm{F}$ (32) due to the nucleus

\footnotetext{
\# An F face is a face having an inward cusp in its gamma-plot.
} 
formation. However this cannot be done explicitly here in the epitaxial case. Furthermore we have to distinguish the Volmer-Weber (VW) and Stranski-Krastanov (SK) cases having very different behaviour.

* For the Volmer-Weber case $\left(\Phi_{\infty}>0\right.$ or $\left.r_{0}>0\right)$ with table II and (32) the nucleation barrier can be factorised [87] as three terms of distinct physical meaning

$$
\Delta F_{V W}^{*} / k T=\left(\Delta F_{\text {hom }}^{*} / k T\right) r_{o} \mathrm{~F}_{r o, K}\left(\Delta \mu / \mathrm{E}_{o}\right)
$$

The first and leading factor is the well known so called homogeneous nucleation barrier (without surface $\beta \rightarrow 0\left(\left(\Phi_{\infty}=0, r_{0}=1\right)\right.$ (see appendix $\left.H\right)$ which is reduced by the second one $r_{0}$, $\left(0<r_{0} \leq 1\right)$ (34) due to substrate wetting. The last term due to epitaxial strain at contrary opposes to the former one since $F_{\mathrm{ro}, \mathrm{K}}<1$ is a function of $\Delta \mu$ which becomes very close to 1 for $\Delta \mu / \mathrm{E}_{0} \approx 10$. Homogeneous nucleation in vapour phase can be estimated to be effective at $\Delta F_{\text {hom }}^{*} / k T \approx 30$ (see appendix $\mathrm{H}$ ) with a critical number of atoms $15<\mathrm{N}^{*}<60$ and driven by supersaturation much higher then $E_{o}($ see $(40)), 6<\Delta \mu / E_{o}<10$. The function $F_{r o, K}$ for these ratios is very close to unity so that the activation barrier for classical nucleation is not influenced by misfit strain!

* In contrast, elasticity is the driving force of the Stranski-Krastanov transition $\left(\Phi_{\infty}<0\right)$ as underlined before. Let us demonstrate it by the following process. The free energy change of the transformation of $z^{\prime} 2 \mathrm{D}$ layers into a $3 \mathrm{D}$ island (volume $\mathrm{V}$ ) sitting on $\mathrm{z}$ layers $\Delta \mathrm{F}^{\prime}$ is that one of (32) $\Delta \mathrm{F}(\mathrm{z}, \mathrm{V})$ minus $\Delta \mathrm{F}\left(\mathrm{z}^{\prime}, 0\right)$ where the $3 \mathrm{D}$ crystals are absent. At constant volume of material A there is for $V / a L^{2}=\theta^{2} h / a<<1$ that means for a small fraction of the film surface covered by $3 \mathrm{D}$ islands :

$$
\Delta F^{\prime}=-\mathrm{E}_{o} V[1-R(r)]+\mid \Phi_{\infty}\left[V-\left(\frac{V}{r}\right)^{2 / 3}\right] e^{-z / \zeta}+4 \gamma_{A}^{\prime} V^{2 / 3} r^{1 / 3}
$$

The activation energy for Stranski-Krastanov transition $\Delta F^{*}(r)$ can thus be obtained by injecting the equilibrium values $\mathrm{V}^{*}$ and $\mathrm{r}^{*}$ of the table I for $\Phi_{\infty}<0$ in the previous relation. For $\Phi_{\infty} e^{-z} \rightarrow 0$ (that makes sense at the SK transition) the barrier reads

$$
\Delta F^{\prime *}(r)=\frac{4}{3} \frac{\left(4 \gamma_{A}^{\prime}\right)^{3}}{\left(3 \mathrm{E}_{o}\right)^{2}} \frac{r^{*}}{\left[1-R\left(r^{*}\right)\right]^{2}}
$$

As usually ([92] and appendix $\mathrm{H}$ ) the activation barrier $\Delta F^{\prime *}(r)$ is proportional to $\gamma_{A}^{\prime 3}$ but $\mathrm{E}_{o}\left(1-R\left(r^{*}\right)\right)$ obviously plays the role of a driving force. On one hand $\Delta F^{*}(r)$ is proportional to $\mathrm{E}_{o}^{-2}$ that means to $m_{o}^{-4}$, on the other hand inhomogeneous relaxation $R(r) \rightarrow 0$ pushes it ${ }^{\S}$. (The lowest value of $\Delta F^{\prime *}(r)$ is roughly reached for $\mathrm{r}^{*}=0.05$ that gives for

\footnotetext{
$\$$ Let us note that in absence of elastic relaxation $(R(r)=1$ in $(50))$ the barrier becomes infinite. Thus clearly again it appears that the inhomogeneous relaxation of the 3D islands is the driving force for Stranski-Krastanov transition.
} 
$\mathrm{Cu}(111)$ where $\gamma \approx 1300 \mathrm{ergcm}^{-2}$ [110] and $\mathrm{E}_{o} / m_{o}^{2}=2.310^{-12} \mathrm{ergcm}^{-3}$ [17] so that $\Delta \mathrm{F}^{*} / \mathrm{kT} \approx 100$ for $\mathrm{m}_{0}=1 \%, \Delta \mathrm{F}^{*} / \mathrm{kT} \approx 30$ for $\mathrm{m}_{0}=2 \%$ but $\Delta \mathrm{F}^{*} / \mathrm{kT} \approx 2$ for $\mathrm{m}_{0}=8 \%$ ). Thus generally Stranski Krastanov transition can only occur for a sufficiently high misfit $\left|m_{o}\right|>2 \%$. Nevertheless some cases of SK growth are well known for misfit of the order of $1.10^{-2}$ where activation barrier calculated from the previous relation seems to be too high. Nevertheless $\Delta \mathrm{F}^{*}$ could be lowered by other mechanisms. Furthermore our box shaped model is not the most flexible one. When considering truncated pyramids (see [65]) the $\gamma_{A}^{\prime}$ in (50) is reduced by some factor, vanishing when the summital facet disappears. This is however also the sign of nonstability of this face even in absence of stress. Furthermore since the true ES is that which minimises the activation barrier, the activation barrier could be lowered by other specific shapes. Last but not least SK transition could start on some point defects so that 2D or 3D nucleation activation barriers are lowered. This is however only possible at very high supersaturation on a F face [92] that means nuclei of several atoms. In fact the problem of the real amount of the activation barrier remains widely an open question.

\subsection{Strain effects in irreversible condensation by growth simulation}

Equilibrium thermodynamics describes statistically nucleation and growth at low supersaturation. Far from equilibrium or really irreversible growth studies are only possible by numerical resolution of kinetical systems [111-113] in the mean field approximation or by simulation catching more or less the collective nature. Latter studies started in their most simplest form by Monte Carlo technics in the $71^{\text {th }}$ [114-116] bringing the now classical and eventually fascinating images of birth, spread and coalescence of islands on a growing compact crystal face, leading to the surface roughening divergence at some critical temperature.

Introduction of elastic strain started only in the $90^{\text {th }}$. Let's report about some studies of Ratsch and Zangwill [117-119] who took a very simple scheme. Atoms are randomly put on the nodes of a quadratic grid with a rate of $\mathrm{F}$ atoms per second per site. When accumulating, eventually at different levels, there is applied the prescription to avoid overhangs and holes in the so generated cubic 3D lattice (solid on solid or SOS model). By this simple scheme column clusters of various shapes and random diverging heights are generated (see [106]). However atoms can move away from the landing site, except to go back to the vapour phase, in the extreme case of complete condensation (in fact the vapour phase is reduced to a "directed beam" so that the lateral faces of the columns don't receive atoms, since no overhangs have to be created). Single atoms migrate to next neighbours sites at the highest rate say $\mathrm{D}$ per second (short range surface diffusion). Usually $\mathrm{D} / \mathrm{F}>>1$ so that surface diffusion is very active. Clusters are not allowed to migrate but they loose single atoms to neighbouring sites with a smaller rate $D \exp (-n \varphi / k T)$, depending on the number $\mathrm{n}=1,2,3,4$ of lateral bonds of strength $\varphi$ which have to be broken ( $\varphi$ stays for an activation energy). This so generated atoms migrate with rate $D$ and fall by chance in traps of $n=1,2,3,4$ bonds $\varphi$ where they reside thus longer thus stronger they are bonded. When all the atoms are bonded vertically by $\mathrm{E}_{\mathrm{s}}=\varphi$ it results normal crystal growth (homoepitaxy) of a flat face $(\mathrm{F})$ that means a new layer starts when the other comes to completion. However single atoms settle also on higher levels thus more F/D is high and multilayer growth may occur. Very flat pyramids (up to 3 layers) may form. Rough kinetics estimations [106,107,113] confirm the mean nucleation density $\ell^{-2}$ and the mean coalescence size $\ell / a=(D / F)^{1 / 4}$. 
Formulating epitaxial growth there is to choice (i) the bond energy $E_{\mathrm{s}} \neq \varphi$, either $\mathrm{E}_{\mathrm{s}}<\varphi$ for VW growth (equivalent to $\Phi_{\infty}>0$ ) or $\mathrm{E}_{\mathrm{s}}>\varphi$ for Frank-van der Merwe and SK growth (equivalent to $\Phi_{\infty}<0$ ); (ii) strain energy has to be accounted too. The studies $[117,118]$ considered SK growth $\mathrm{E}_{\mathrm{s}}>>\varphi$ and due to the model of first neighbours interactions, only one SK wetting layer A is grown on the substrate B (see also our analysis close to (38) and appendix E). The atoms in the second layer are vertically bonded with $\mathrm{E}_{\mathrm{s}}=\varphi$ so that lateral layer-by-layer growth should follow. Due to misfit however the SK layer is strained and strain weakening of lateral bond energies is taken as guiding principle by the authors:

$$
\frac{1}{2} 4 \varphi \rightarrow 2 \varphi-\mathrm{E}_{o}
$$

However 3D box shaped clusters are elastically relaxed by $R(h / \ell) \propto(\ell / h)^{1 / 2}$ (a crude approximation of figure 7) so that the frequency prescription for the growth simulation is $D \exp \left[-n\left(\varphi-\mathrm{E}_{0} R(r) / 2\right)\right]$ for detaching an atom on a summital layer of a cluster [119]. Atoms thus detach more frequently a cluster is flat, so that taller cluster are favoured during the evolution; this trend being thus stronger the misfit square is high. Simulated images $[117,118]$ show slightly dispersed rectangular near quadratic based clusters with mostly complete layers. At increasing total coverage above the SK layer of $\mathrm{Ft}=1 / 4,1 / 23 / 4$ monolayers, single and double layered clusters appear progressively. At Ft=1, 2D islands of 2,3, and 4 layers are formed so that only some half of the SK layer is covered. This thickening of the clusters is clearly due to the strain relaxation $\mathrm{E}_{0} R$ even if somehow exaggerated by the type of simulation.

The authors observed that thickening starts only at "some critical misfit" of $3 \%$. This surprising result may not be general and needs our comments. Consider a distribution of box shaped crystals above their SK wetting layers so that the energy to spent is in average, that to create lateral faces plus the elastic energy written in the former mentioned approximation $\left(R^{3 D}(r) \approx 0.14 / \sqrt{r}\right.$ see 2.2.3.2.)

$$
\Delta F=4 \gamma_{A} h \ell+0.14 \mathrm{E}_{o} V \sqrt{\ell / h} \equiv 4 \gamma_{A} V / \ell+0.14 \mathrm{E}_{o} \sqrt{V \ell^{3}}
$$

At constant volume $V=h \ell^{2}$ for spontaneous thickening there must be $\partial \Delta F /\left.\partial h\right|_{V}<0$. This is realised when for this volume $\ell / a>\left[19 \gamma_{A} / \mathrm{E}_{\delta} a\right]^{2 / 3}(h / a)^{1 / 3}$. Doubling the height of a $2 \mathrm{D}$ island occurs when its size exceeds $\ell /\left.a\right|_{1}>\left[19 \gamma_{A} / \mathrm{E}_{0} a\right]^{2 / 3}$. Clearly it has to be smaller than the coalescence size $\ell /\left.a\right|_{c}=(D / F)^{1 / 4}$ (if not the single height islands annihilate mutually) the authors simulations took $\mathrm{D} / \mathrm{F}=10^{6}$ so that $\ell /\left.a\right|_{c} \approx 30$ atomic units. There is according to Frank's rule $[45] \gamma / E \approx 10^{-9} \mathrm{~cm}$ for clean crystal faces, but $2 \mathrm{D}$ edges being thermally roughened we take $\gamma_{1} / E \approx 1 / 3.10^{-9} \mathrm{~cm}$ so that with $\mathrm{a}=3 \AA$ there is a critical size $\ell /\left.a\right|_{c} \approx 170$ (for $\left.\mathrm{m}_{\mathrm{o}}=1 \%\right), 40(3 \%), 26(4 \%), 15(6 \%)$. Thus doubling can only start for misfit greater than roughly $3 \%$ which corresponds to what the authors "observed". Conversely it can be foreseen that "SK roughening " can be avoided when $\ell_{c}<\ell_{1}$ that means by decreasing the coalescence 
size or increasing the density of critical nuclei. This can be done by increasing the reduced flux $\mathrm{F} / \mathrm{D}>60 \mathrm{~m}_{o}^{16 / 3}$ or by increasing the nuclei density by other means. Lets add that these simulations have been done on a flat $\mathrm{F}$ face. When done on a vicinal with terraces width $\mathrm{L}$, the coalescence size has an upper limit for $2 \mathrm{D}$ islands $<\mathrm{L}$.

\subsection{Growth instability induced by strain on vicinal faces}

\subsubsection{Growth of a vicinal face without strain}

Growth instabilities are very frequent in bulk growth when diffusion-convexion of matterheat are involved. In vapour growth, even outside any strain considerations, surface diffusion coupled with interfacial kinetics leads to instabilities we have to mention first. In the pioneer work of BCF [105] a vicinal face receives (or looses) adatoms from the terraces where they migrate towards (or away) the steps making them to advance or to recede. In any case, steps exchange (from their kinks) their atoms with the two adjacent terraces from the top side (+) and from the low side (-) with frequencies $\mathrm{D}^{+}$and $\mathrm{D}^{-}$leading to a very quiet step flow when $\mathrm{D}^{+}=\mathrm{D}^{-} \leq \mathrm{D}$. Disymmetric step kinetics may result from adsorption of impurities in the kinks $[105] \mathrm{D}^{+} \neq \mathrm{D}^{-} \leq \mathrm{D}$. But there exists also some intrinsic effects for clean steps with $\mathrm{D}^{+}<\mathrm{D}^{-} \leq \mathrm{D}$ due to some activation barrier near the upper ledge, the so-called Schwoebel [120]- Erhlich [121] barrier $^{* * * *}$.

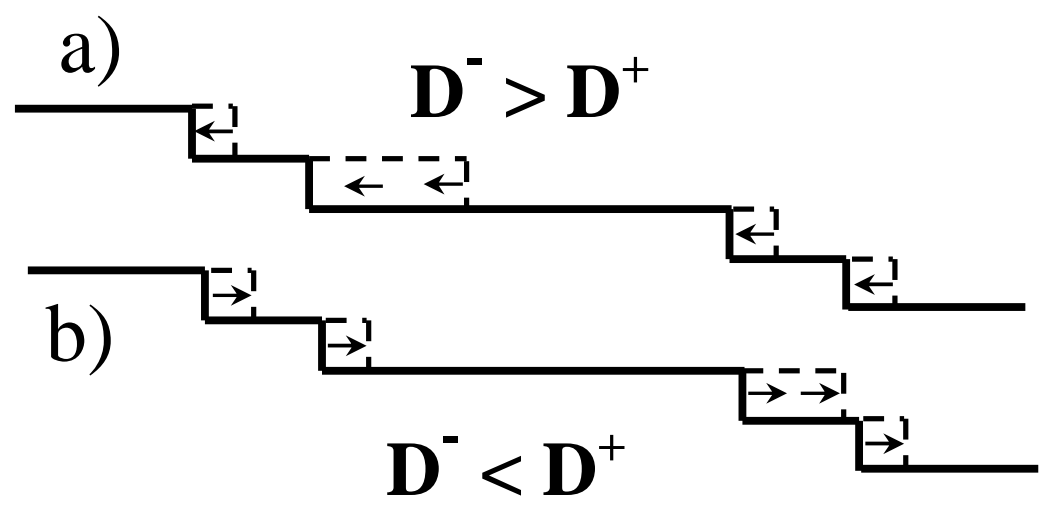

Figure 14: a/ Adatom sticking is easier onto an upper step $\left(D^{-}>D^{+}\right)$. Then since the number of atoms that reach a terrace is proportional to the terrace area, a terrace larger than its neighbours becomes smaller, thus all terraces reach the same size, each step reaches the same velocity and step flow mechanism occurs.

b/ Adatom sticking is easier onto a lower step $\left(D^{-}<D^{+}\right)$, a terrace larger than its neighbours becomes larger and larger and step bunching occurs. The opposite scenarios are valid for evaporation. This concerns a non-strained solid.

In BCF's theory the lateral step velocity is an increasing function of the size of the adjacent upper and lower terraces: $\mathrm{v}=D^{+} f\left(L^{+}\right)+D^{-} f\left(L^{-}\right)^{\dagger \dagger \dagger}$. Suppose a given step (figure 14) in a

\footnotetext{
*** When atoms approach or leave an upper ledge second attractive neighbour bonds have to be cut, but those from the lower ledge not.

${ }^{t+\dagger}$ In fact $\mathrm{v}$ depends upon the normalised distances $\mathrm{L} / 2 \mathrm{x}_{\mathrm{s}}$, where $\mathrm{x}_{\mathrm{s}}$ is the mean diffusion distance before desorption of an atom.
} 
regular train $\mathrm{L}^{+}=\mathrm{L}^{-}=\mathrm{L}$, during growth the mean speed $\overline{\mathrm{v}}>0$ fluctuates, say $\Delta \mathrm{L}>0$ so that the lower terrace becomes smaller, the upper one wider. The velocity of this step is

$$
\mathrm{v}=\overline{\mathrm{v}}+\Delta \mathrm{v}=\mathrm{D}^{+} f(L+\Delta L)+D^{-} f(L-\Delta L) \approx\left(D^{+}+D^{-}\right) f(L)+\left.\left(D^{+}-D^{-}\right) \frac{d f}{d L}\right|_{L} \Delta L
$$

The symmetric case $\mathrm{D}^{+}=\mathrm{D}^{-}$doesn't change the velocity from the mean value of the train, $\mathrm{D}^{+}<\mathrm{D}^{-}$slows down the step, $\mathrm{D}^{+}>\mathrm{D}^{-}$boosts it and may lead to instability. For evaporation, $\overline{\mathrm{v}}<0$, the opposite happens.

P.Bennema and G.H.Gilmer [122] showed that the governing differential equation is similar to that of a chain of masses connected by springs, having for growth (evaporation) an exponential damping regime for $\mathrm{D}^{+}\left\langle\mathrm{D}^{-}\left(\mathrm{D}^{+}>\mathrm{D}^{-}\right)\right.$and an exponentially increasing perturbation for $\mathrm{D}^{+}>\mathrm{D}^{-}\left(\mathrm{D}^{+}<\mathrm{D}^{-}\right)$leading to step bunching (see also [106]). In summary ordinary (usual) Schwoebel-Ehrlich effect renders, fortunately, normal crystal growth stable.

The case of strained layers of vicinal nature has been studied by Duport et al. in 1994 [123,124] and Tersoff in 1995 [125]. Because of the height $h$ discontinuities, the border of these strained layers bear elastic monopoles in excess (see (16)) with the elastic dipoles existing without strain (see 2.). There is first to study the thermodynamic stability of such a train, secondly its stability versus growth or evaporation.

\subsubsection{Tersoff's step bunching driven by step-step interaction due to bulk strain}

(i) We have seen (1.3.2.) that a step on a vicinal surface can be described as a line of dipoles at which adds a line of monopoles. The interaction energy per unit length of a pair of identical, L-apart, steps $(\mathrm{h}=\mathrm{a})$ can thus be separated in four terms. Each of these terms and its physical origin are described in table III when surface stress is neglected $\left(F_{3}=0\right.$ in (14))

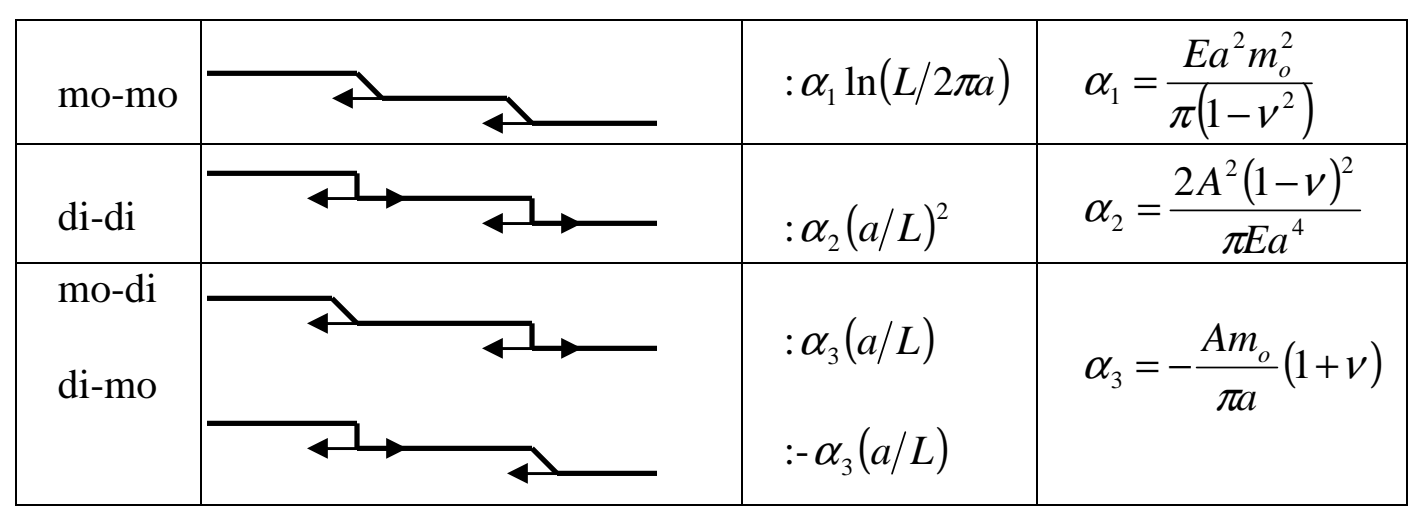

Table III: Sketch of the various interactions in between elastic monopoles (mo) and dipoles (di), with the analytical expressions of the interaction energies in column 3. Here $m_{o}<0$ and $A>0$

In the given analytical expressions of the four terms (see third and fourth column of table III) let us recall that $\mathrm{A}$ is a dipole moment as in I32. Here $\mathrm{A}>0$ describes a dilatation centre. Identical monopoles are said long range attractive $(\ln L)$ whereas the identical dipoles, whatever their sign, are said short-range repulsive $\left(\mathrm{L}^{-2}\right)$. The medium range monopoles- 
dipoles interaction depends on the sign of $\mathrm{Am}_{\mathrm{o}}$, but for the pair of steps the total interaction is zero. In appendix $\mathbf{J}$ we derive these interactions.

Consider now a step $\mathrm{m}$ at position $\mathrm{x}_{\mathrm{m}}$ in between two others steps (of same sign) at $\mathrm{x}_{\mathrm{m}-1}$ and $\mathrm{x}_{\mathrm{m}+1}$, with $\mathrm{x}_{\mathrm{m}+1^{-}} \mathrm{x}_{\mathrm{m}-1}=2 \bar{L} \quad\left(\mathrm{x}_{\mathrm{m}}\right.$ is the deviation of step $\mathrm{m}$ from its middle position taken as origin see figure 15). The step-step interaction per unit length versus the deviation $\mathrm{x}_{\mathrm{m}}$ from table III thus reads

$$
U^{s-s}\left(x_{m}\right)=U_{1}\left(x_{m}\right)+U_{2}\left(x_{m}\right)=\alpha_{1} \ln \left[\frac{L-x_{m}^{2}}{(2 \pi a)^{2}}\right]+\alpha_{2}\left[\left(\frac{a}{L-x_{m}}\right)^{2}+\left(\frac{a}{L+x_{m}}\right)^{2}\right]
$$

and is drawn as a full line on figure 15 . The step $m$ at $x_{m}=0$ thus is mechanically unstable since by some fluctuation it is attracted either on one side or the other side and finally trapped in the left or right minimum due to the repulsive potential. For figure 15 we took usual values of the various ingredients $\mathrm{E}=5.10^{11} \mathrm{erg} \mathrm{cm}, \mathrm{v}=1 / 3, \mathrm{a}=2.10^{-8} \mathrm{~cm}$. For the dipole moment $\mathrm{A}=0.7 \mathrm{eV}=1.10^{-12} \mathrm{erg}$ consistent with $\mathrm{E}$ and a (see appendix I). With a misfit $\mathrm{m}_{\mathrm{o}}=2.10^{-2}$ one thus obtains $\alpha_{1}=2.10^{-8} \mathrm{erg} \mathrm{cm}^{-1}, \alpha_{2}=1.10^{-5} \mathrm{erg} \mathrm{cm}^{-1}$ and $\alpha_{2} / \alpha_{3}=1 / 3.10^{3}$. From figure 15 where $\mathrm{L} / \mathrm{a}=100$ the pairing distance is seen to be $\left(\mathrm{L}-\mathrm{X}_{\mathrm{m}}\right) / \mathrm{a}=23$ atomic units. A good approximation is $\left(2 \alpha_{2} / \alpha_{1}\right)^{1 / 2} \approx 2|A| /\left(E a^{3}\left|m_{o}\right|\right)=25$. From latter result one understands that epitaxial strain $\mathrm{m}_{\mathrm{o}}$ whatever its sign compresses the pair. Adding to $\alpha_{2}$ a $\alpha_{2}(\mathrm{~T})$ term ${ }^{\ddagger \ddagger}$, a temperature increase may further separate the pair.

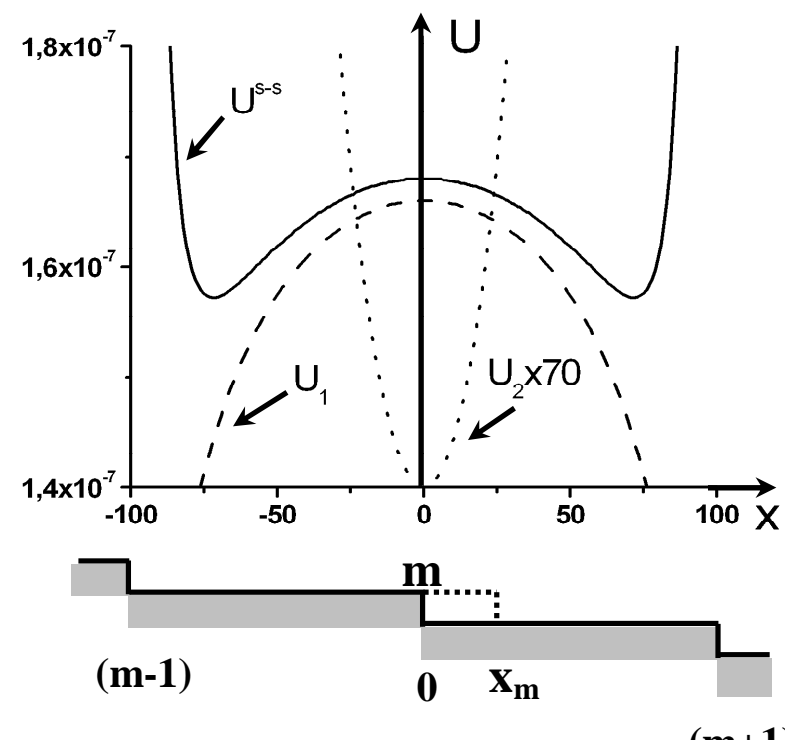

Figure 15: Interaction energy of a step of a strained solid $U^{s-s}(54)$ versus its the position in between two others, $U_{1}$ attracting part due to elastic monopoles, $U_{2}$ repulsive part due to elastic dipoles (units: $\mathrm{erg}^{-1}$ ).

$(\mathbf{m}+1)$

\footnotetext{
$\$$ The elastic interactions as all elastic properties are slightly decreasing with temperature. An independent dipole-dipole repulsion $\alpha_{2}(T)(a / L)^{2}$ exists for $\mathrm{T} \neq 0$ due to kink formation [105] so that the steps meander and due to their mutual confinement there results [126] an exponentially increase of $\alpha_{2}(\mathrm{~T})$ with $\mathrm{T}$. Obviously these "dipoles" don't couple with elastic monopoles or dipoles.
} 
(ii) How does this mechanical instability lead to step bunching? This is a matter of cooperative kinetics of transport of atoms by surface diffusion in between steps. Tersoff [125] considers the adatoms coming from exchange with the kinks on the steps and eventually from an incoming flux $\mathrm{Fsec}^{-1}$ per site. No atom leaves the crystal, complete condensation is thus supposed so that the time dependant adatom density $\theta$ is governed by the simplified BCF [106] diffusion equation. The boundary conditions at the steps are simplified too: (1) The transfert of atoms from or to the kinks and nearest adatoms positions $\mathrm{x}_{\mathrm{m}} \pm \mathrm{a}$ has no kinetical barrier (at least that of the usual surface diffusion coefficient D). There is no interfacial kinetics, no Schwoebel or other retardation effects. (2) The local adatom density at the steps is that of thermodynamical equilibrium

$$
\theta\left(x_{m}+a\right)=\theta\left(x_{m}-a\right)=\exp \left[-\left.\Delta E\right|_{x_{m}, \bar{L}} / k T\right\rfloor
$$

where $\Delta \mathrm{E}$ is the bond energy of a kink atom minus that one of the adjacent adatom at $\pm \mathrm{a}$ (in fact the adatom creation energy from a kink atom). This quantity is however modified by the elastic field where the kink is located at $\mathrm{x}_{\mathrm{m}}$ inside the train of steps $\bar{L}$ as quoted in (55) by the indices $\mathrm{x}_{\mathrm{m}}$ and $\bar{L}$. A kink atom at $\mathrm{x}_{\mathrm{m}} \neq 0$ is submitted to a net attractive force $a f\left(x_{m}\right)=-a \partial U^{s-s} /\left.\partial x\right|_{x_{m}}>0$ towards the nearest of its neighbouring step. When gaining an atom the kink goes ahead by $\Delta \mathrm{x}_{\mathrm{m}}=\mathrm{a}$ so that its energy changes by $\Delta W \approx a f\left(x_{m}\right) \Delta x_{m}=a^{2} f\left(x_{m}\right)$ so that $\$ \S \S$

$$
\left.\left.\Delta E\right|_{x_{m}, \bar{L}} \approx \Delta E\right|_{0, \bar{L}}-\left.a^{2} \frac{\partial U^{s-s}}{\partial x}\right|_{x_{m}}
$$

where now $\left.\Delta E\right|_{0, \bar{L}}$ is the adatom creation energy from a kink when this force vanishes. From (55) and (56) there is

$$
\theta\left(x_{m} \pm a\right)=\theta( \pm a) \exp \left[-a^{2} \frac{\partial U^{s-s} /\left.\partial x\right|_{x_{m}}}{k T}\right]
$$

which means that these elastic interactions due to epitaxial strain boost the equilibrium adatom density at the step when the step deviates from its middle position. This is a simple way to formulate how a mechanical effect is transformed in a chemical one.

For testing stability let us consider at the time $\mathrm{t}=0$ a regular train of steps of mean distance $\bar{L}$. Let us assume a small displacement of every N steps by $u_{m}(o)=\Delta \cos (2 \pi m / N)$, at a small time $\mathrm{t}>0$ the following time evolution (58) is found to be valid [125]

$$
u_{m}(t) \approx \Delta e^{r t} \cos \left[\frac{2 \pi}{N}(m+F t)\right]
$$

\footnotetext{
${ }^{\S \S}$ In fact this is only true when each step site is a kink site. When the kink density is $0<\varepsilon<1$, the local displacement is reduced to $\Delta \mathrm{x}_{\mathrm{m}}=\mathrm{a} \varepsilon$
} 
The exponent is given in (59) for widely spaced steps $\left(\mathrm{L} / \mathrm{a}>\alpha_{2} / \alpha_{1}\right)$ and since positive, leads to an amplification of the bunching rate $r$ we factorise:

$$
r=\frac{E a^{3} m_{o}^{2}}{1-v^{2}}\left(a^{2} D_{M} / 2 k T\right)\left[\left(\frac{2 \pi a}{N \bar{L}}\right)^{3} \pi\left(1-\frac{1}{N}\right)\right]
$$

First there is the "driving energy" of epitaxial strain per atom, then the "kinetic resistance" due to the material transport. Appears the mass diffusion coefficient

$$
D_{M}=D \exp \left(-\left.\Delta E\right|_{x_{m}, \bar{L}} / k T\right)=D_{o} \exp \left(-\left(\left.\Delta E\right|_{x_{m}, \bar{L}}+E^{*}\right) / k T\right)
$$

composed of the adatom creation energy $\left.\Delta E\right|_{x_{m}, \bar{L}}$ usually greater than the activation barrier $\mathrm{E}^{*}$ for surface diffusion of atoms. $\left.\Delta E\right|_{x_{m}, \bar{L}}$ is able to freeze this rate process when temperature is not high enough, e.g. when $\mathrm{T}<2 / 3 \mathrm{~T}_{\text {melting }}$. The last term in (59) shows that bunching starts for $\mathrm{N}=2$, that puts in evidence the cooperative nature of the process. $\mathrm{N}=2$ is the most efficient mode, the amplification slows down rapidly for modes $N>2$. This initial bunching rate is flux independent. At $\mathrm{F}=0$ (but zero evaporation is prescribed) numerical resolution [125] showed, starting with a random train, the development of the bunches with time. The bunch size (mean number of steps in a bunch) varies monotonously as $\langle\mathrm{n}\rangle \alpha \mathrm{t}^{1 / 4}$. Atomically flat zones can be obtained separated by bundles of many steps. Curiously it was observed (but not systematically studied) that when flux is put on, maintaining the same other conditions (1) bunching progresses less rapidly $\langle\mathrm{n}\rangle \propto \mathrm{t}^{1 / 6}(2)$ bunching saturates at some small value $\langle\mathrm{n}\rangle_{\text {sat }}=3$ for $F=25$. Such simulations should be reactivated in parallel with in situ experiments similar to those of Métois et al. [127] under well controlled flux $\mathrm{F}<0, \mathrm{~F}=0$ or $\mathrm{F}>0$.

\subsubsection{Duport's strain driven surface diffusion instability}

The Grenoble group [123,124] predicted first in 1994 an other instability we are now able to qualify more precisely. The phenomenon concerns strained vicinal faces under biaxial misfit $\mathrm{m}_{\mathrm{o}}$ with an incoming flux $\mathrm{F}>0$ but without re evaporation (complete condensation) as in 3.3.2.

The authors considered the elastic interaction of an adsorbed atom on a terrace in-between two consecutive $\mathrm{L}$ apart steps (see figure 16) described as a dipole $\mathrm{A}^{\text {ad }}$ located in-between two lines of identical monopoles $\mathrm{m}$ on the right and $\mathrm{m}-1$ on the left. Taking the middle point as origin of $\mathrm{x}_{\mathrm{ad}}$, the interaction energy per atom reads in absence of surface stress and thus with $\mathrm{F}_{3}=0$ in (14) (see table III)

$$
U_{L}^{a d-s}\left(x_{a d}\right)=U_{3}\left(x_{a d}\right)+U_{2}\left(x_{a d}\right)=\alpha_{3}^{\prime}\left[\frac{a}{L / 2+x_{a d}}-\frac{a}{L / 2-x_{a d}}\right]+\alpha_{2}\left[\left(\frac{a}{L / 2+x_{a d}}\right)^{2}+\left(\frac{a}{L / 2-x_{a d}}\right)^{2}\right]
$$


where $\quad \alpha_{3}^{\prime}=-\frac{A^{a d} m_{o}}{\pi a}(1+v) \quad[123,124]^{* * * *}$ for the dipole-monopoles interaction and $\alpha_{2}^{\prime}=2 A^{a d} A /\left(\pi E a^{3}\right)$ for the dipole-dipoles interaction (see table III). In principle $\mathrm{A}^{\mathrm{ad}} \neq \mathrm{A}$ but are of same sign so this interaction again is repulsive. Mostly $\left|\mathrm{A}^{\mathrm{ad}}\right|<|\mathrm{A}|$.

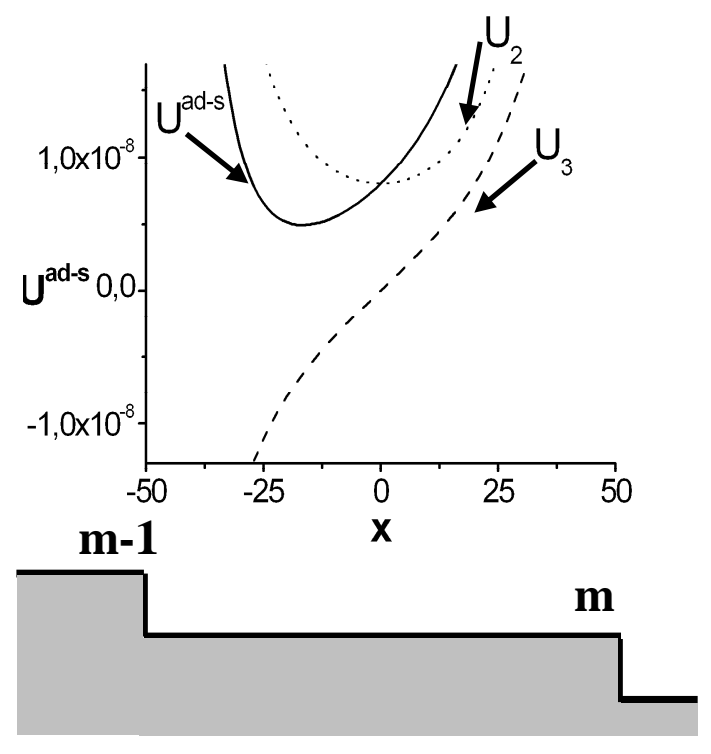

Figure 16: Interaction energy $U^{a d-s}$ of (61) erg $\mathrm{cm}^{-1}$ of an adatom (dipole) and two steps of same sign of a strained body. $U_{2}$ repulsive part due to the two dipole-lines with the addipole interaction, $U_{3}$ attractive part due to the two monopole-lines and the ad-dipole

In figure 16 we draw (61) as a full line taking the same numerical values as in figure 15 of $\mathrm{E}, \mathrm{v}, \mathrm{a}, \mathrm{m}_{\mathrm{o}}, \mathrm{L} / \mathrm{a}$ and $\mathrm{A}$, furthermore we put $\mathrm{A}^{\mathrm{ad}}=\mathrm{A}$ (making therefore an underevaluation). Thus $\alpha_{3}=8.4 .10^{-15}, \alpha_{2}=2.10^{-13}$ erg per adatom. For $\mathrm{A}^{\mathrm{ad}} \mathrm{m}_{\mathrm{o}}>0$ the adatom is attracted to the lower terrace of the steps the short range repulsion creating a single well located near the lower terrace of the step, (specifically here $\mathrm{x}_{\mathrm{ad}} /(\mathrm{L} / 2)=-20$ atomic units). When $\mathrm{A}^{\text {ad }} \mathrm{m}_{\mathrm{o}}$ changes sign, the well switches to the symmetrical position. The stationary adatom density at the position $\mathrm{x}$ along the terrace is given by the resolution of

$$
F-\frac{\partial J_{m}(x)}{\partial x}=0
$$

where $J_{m}(x)$ is the net current proportional to the gradient of adatom density $\theta(x)$ to which is added a drift term due to the elastic force $f_{a d}=-\partial U^{a d-s} / \partial x$ acting on the adatoms:

$$
J_{m}(x)=-D \frac{\partial \theta}{\partial x}+\frac{D}{k T} \theta(x) f_{a d}
$$

where $\mathrm{D} / \mathrm{kT}$ is the so-called Einstein mobility of the adatoms when submitted to the force $f_{a d}(x)$.

The Einstein-Focker-Planck equation (63) thus gives a purely kinetics effect as can be easily seen since when this force derives from the equilibrium distribution $\theta(x)=\exp \left[-U^{a d-s} / k T\right]$

\footnotetext{
***** We put here minus sign since our misfit convention is of opposite sign of Duport's $\delta \mathrm{a} / \mathrm{a}=-\mathrm{m}_{\mathrm{o}}$.
} 
there will be no current on the terraces. Putting (63) in (62) and integrating along a terrace gives

$$
F x+D\left[\theta(x)+\int \frac{\theta(x)}{k T} \frac{\partial U^{a d-s}}{\partial x} d x\right]+C=0
$$

The constant $\mathrm{C}$ is obtained from boundary conditions at the step. Obviously they have to be non-equilibrium conditions: for the left step (m-1), $\frac{D^{-}}{a} \theta(L / 2)=C-F L / 2$; for the right step $\frac{D^{+}}{a} \theta(L / 2)=C+F L / 2$. The condition for a step $\mathrm{m}$ to collect from its front and its back terrace leads again to a differential equation very similar to (59) with a stability exponent $r$

$$
r=-\left\{\left(\frac{D}{D^{+}}\right)^{2}-\left(\frac{D}{D^{-}}\right)^{2}+\frac{2(1+v)}{\pi} \frac{A^{a d} m_{o}}{k T} \frac{L}{a}\right\}\left(\frac{2 \pi a}{N L}\right)^{2} F
$$

Physically however the result is very different. (i) The oscillation of the train suffers damping or amplification only when a flux $\mathrm{F} \neq 0$ exists (clearly only an incoming flux since evaporation was precluded). (ii) The shortest mode, even $\mathrm{N}=1$, is the most efficient one. (iii) For $m_{0}=0$, an asymmetric adatom integration $\left(D^{+}<D^{-}<D\right)$ is stabilising (similar to Schwoebel effect). For $A^{\text {ad }} m_{0}>0$ (for self-adsorption $A^{\text {ad }}>0$ it means $m_{o}>0$ ) stability is increased even for symmetric integration $\left(D^{+}=D^{-}\right)$. (iV) For the opposite case $A^{\text {ad }} m_{0}<0$ (that means $m_{0}<0$ for self adsorption) and with still $\mathrm{D}^{+}<\mathrm{D}^{-}<\mathrm{D}$, the epitaxial strain drives so much the adatoms downward the step that they overcome easily the Schwoebel barrier. However this only happens provided the mean step distance L/a overpasses a critical value depending on the height of the barrier. ${ }^{\dagger \dagger \dagger \dagger}$

From our discussion here and the one in III32 it is clear that Duport's and Tersoff's instabilities are not of the same nature. The Tersoff's one is misfit square dependent, exists at zero flux and slows down for increasing flux. The Duport's one is misfit sign dependent, does not exist for $m_{o}>0$ and when exists $\left(m_{o}<0\right)$ is boosted by increasing flux. This gives a contradictory feeling about both effects. Furthermore in Tersoff's theory the adatoms have not been supposed subjected to the elastic field of the steps and thus are not dragged towards the steps. In Duport's theory the steps are supposed do not interact by their elastic field as of course they should. The situation is however not so bad since Tersoff's and Duport's instabilities in fact occur in different temperature ranges. Indeed in Tersoff's formula (59) appears the mass diffusion coefficient $D_{M}=D_{o} e^{-E^{*} / k T} e^{-\Delta E / k T}$, which is very temperature dependent through $\Delta \mathrm{E} / \mathrm{kT}$, and thus only works at very high temperature. On the contrary, in Duport's formula (65) appears the surface diffusion coefficient $D=D_{o} e^{-E^{*} / k T}$. Thus since $\mathrm{E}^{*} / \Delta \mathrm{E} \approx 1 / 5$ for stable faces the Duport instability works at low temperature where kinks can't produce adatoms by their own (Adatoms in this case are only provided by the incoming flux).

\footnotetext{
${ }^{\dagger+\dagger \dagger}$ Thus wafers with very small miscuts and the use of very weak flux would be helpful for avoiding this instability.
} 


\subsubsection{Miscellaneous kinetics effects}

* In the previous section we have seen that strain can modify surface diffusion by way of the supplementary elastic forces along the steps acting on adatoms. Nevertheless strain can also have an effect on the diffusion coefficient itself as shown by Schroeder and Wolf [128] who calculated activation barriers for diffusion on strained high symmetry plane surfaces (without steps) of simple cubic, fcc and bcc crystals. For this purpose they described pair-wise interactions by means of an anisotropic Lennard-Jones potential with a strain-modified distance in between atoms; then they placed an adatom on a binding site and moved it by small steps. The activation energy for surface diffusion is calculated by a conventional minimal energy path saddle point. The main result is that generally for tensile stress $(\sigma>0)$ the diffusion barrier is increased whereas compressive stress $(\sigma<0)$ decreases the barrier. (see figure 17). The diffusion barrier change is mainly due to a change of the saddle point energy whereas the minima are shifted only very little. As said by the authors this behaviour can be naïvely understood on the basis of limiting cases. Indeed in the limit of large compressive stress $(\sigma<0)$ the surface becomes continuous and thus there is no longer a diffusion barrier. On the contrary, within the limit of large tensile stress $(\sigma>0)$ the surface consists of isolated atoms and diffusion becomes equivalent to breaking a pair of atoms and building a new pair ${ }^{+1+}$. In the same paper the authors have also studied theoretically diffusion on top of a stressed island. In this case, since the finite size island can elastically relax by its free edge the strain along the top surface of the island becomes inhomogeneous and thus diffusion may vary from the centre of the island towards its edges. For compressive strain the diffusion is faster near the island centre whereas for tensile stress it is faster towards the edges. Thus it should be easier to nucleate on top of a tensile strained island than on top of a compressive strained island ${ }^{\S \S \S}$.

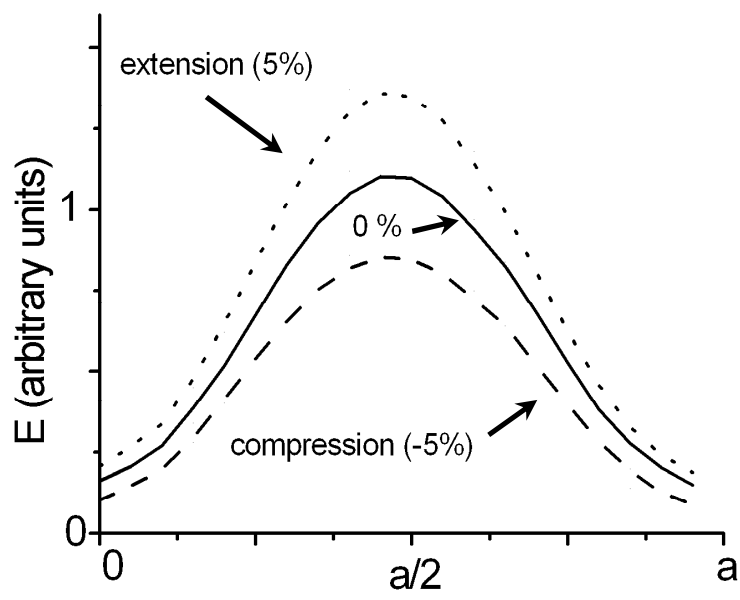

Figure 17: Diffusion barrier path for an adatom on an homogeneously strained cubic lattice (parameter a) calculated by [128]. Upper curve: tensile strain (5\%), middle curve $\mathrm{m}_{0}=0$, lower curve: compressive strain (-5\%). In fact for a homogeneous strained (001) surface of a simple cubic crystal the diffusion barrier depends linearly upon strain which can be easily shown by a simple first order development of pair-wise potential in respect to strain [128].

These two effects are second order effects in comparison to elastic interaction in between adatoms and steps as described in the previous section. Furthermore the simple surface diffusion change cannot modify the growth mechanism. More precisely since adatom density essentially depends upon the ratio D/F a strain-induced increase (decrease) of the surface

\footnotetext{
$\$+\$$ In other words in this latter case the diffusion barrier becomes equal to the pair-binding energy

$\S \S \S$ More precisely for an inhomogeneous strained surface the saddle point energy and the binding energy may vary as well. The saddle point energy change leads to an inhomogeneous nucleation whereas the binding energy change leads to a drift term in the diffusion current. Nevertheless for cubic crystals the binding energy change remains weak [128].
} 
diffusion constant (D) is exactly equivalent to an appropriate increase (decrease) of flux (F) and thus may only weakly shift the transition between step flow and 2D nucleation or change the nucleation density. At the same since the taller a crystal, the more relaxed its top face, this kind of elasticity-induced Schwoebel barrier thus can only help the first stages of the thickening of tensile islands.

* We avoided in these lectures to treat alloy formation and especially the effect of strain. Let's fisrt mention some facts.

(i) Deposition of A pure on a B pure substrate forming either 3D crystals (VW) or thin epitaxial layers (F-vdM). In the absence of epitaxial misfit and defects the interface in both cases moves, in a planar way in the first case, rebuilding the substrate in the vicinity of the 3D crystals for the second case. One speaks about compositional strain due to the different atomic radius of $\mathrm{A}$ and $\mathrm{B}$.

(ii) Deposition of an alloy $A_{x} B_{1-x}$ on a substrate $B$. Interface diffusion is common for planar $\mathrm{Ge} / \mathrm{Si}$ or $\mathrm{Si} / \mathrm{Ge}$ systems and starts at temperature higher than $650^{\circ} \mathrm{C}$ but is difficult to follow. Some other systems are more accessible and much more brilliant for demonstration. It is the case of epitaxial deposit $\mathrm{BaTiO}_{3}$ film deposited on $\mathrm{MgO}$ buffered sapphire substrate: $\mathrm{BaTiO}_{3}(100) / / \mathrm{MgO}(100) \mathrm{Al}_{2} \mathrm{O}_{3}(11 \overline{2} 0)$ [129] where the interface $\mathrm{BaTiO}_{3} / \mathrm{MgO}$ is very abrupt whereas $\mathrm{MgAl}_{3} \mathrm{O}_{4}$ layers appear at the interface $\mathrm{MgO} / \mathrm{Al}_{2} \mathrm{O}_{3}$ (see figure 18). This alloy layer thickness depends on the subsequent deposition time of $\mathrm{BaTiO}_{3}$ deposited at $1100^{\circ} \mathrm{C}$, the $\mathrm{MgO}$ layer having been deposited on $\mathrm{Al}_{2} \mathrm{O}_{3}$ at $650^{\circ} \mathrm{C}$ only.

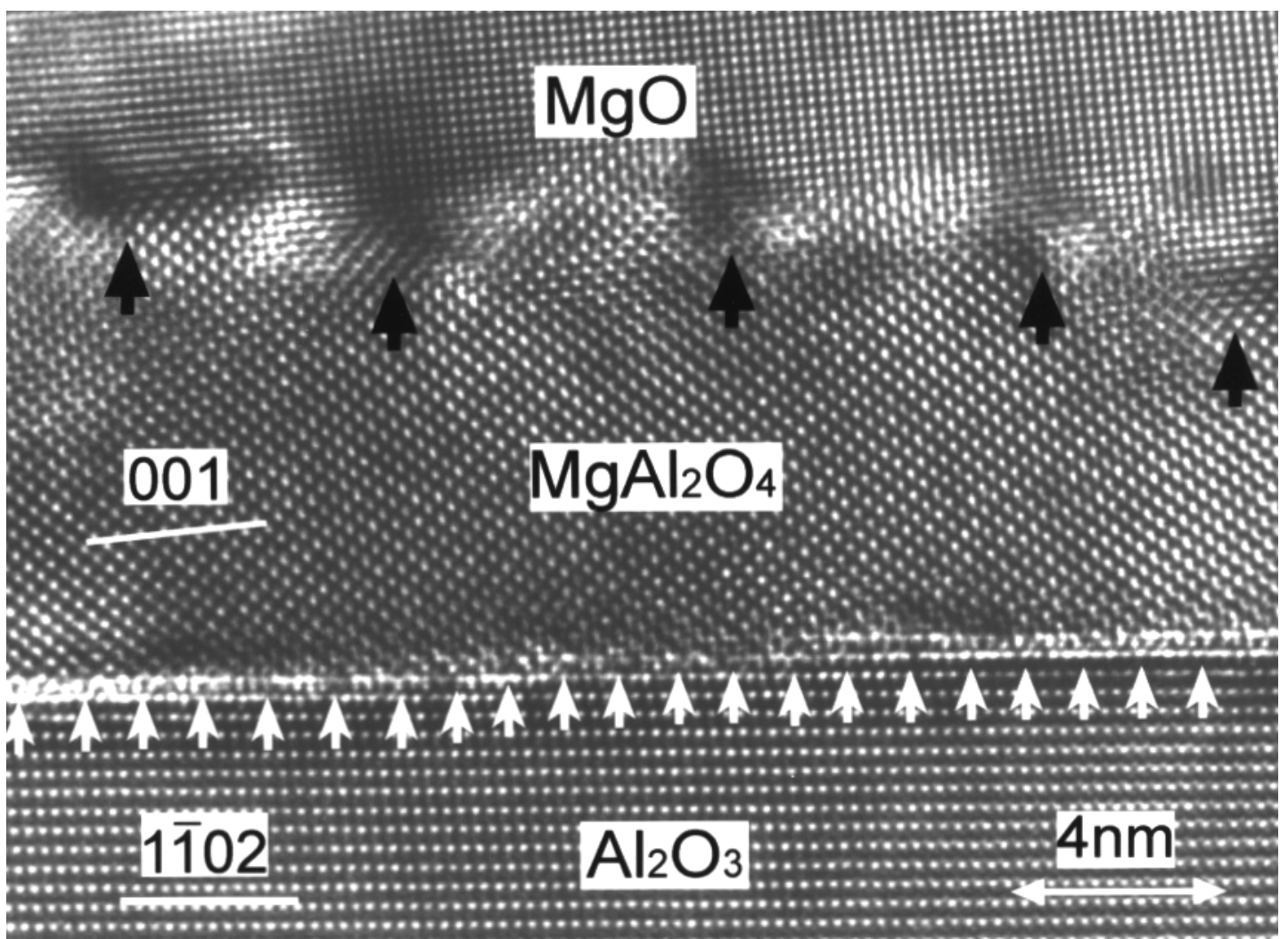

Figure 18: $\mathrm{BaTiO}_{3}(100) / / \mathrm{MgO}(100) \mathrm{Al}_{2} \mathrm{O}_{3}(11 \overline{2} \mathrm{O})$ : Lattice image of the $\mathrm{MgAl}_{2} \mathrm{O}_{4}$ spinel reaction layer between the $\mathrm{MgO}$ buffer layer and the sapphire substrate (courtesy of C.H. Lei et al [129]) 
(iii) Interesting to study is the surface of a strained alloy where compositional and morphological instabilities occur both [130-141]. Undulations become totally unstable for all wavelengths. Islands nucleate at different composition than the alloy layer, stress induced nucleation rate is drastically increased. Experiments are far behind theory.

Last but no least lets mention very important practical but trivial effects: misfit changes that occur at the end of a growth process when temperature comes back to normal temperature and the different dilatation coefficient are not adjusted.

\section{A FEW REMARKS ABOUT INTERACTING CRYSTALS}

Up to now we have only described isolated epitaxial crystals. Generally one has to do with a collection of crystals so that when their mean distance $\bar{L}$ approaches their mean size $\bar{\ell}$, these crystals may interact. We will distinguish two types of interactions. Even when the crystals are far one from another, $\bar{\ell}<<\bar{L}$, they may exchange atoms by surface diffusion on the substrate provided the surface diffusivity is high enough. At smaller distances $\bar{\ell} \approx \bar{L}$, there is furthermore to consider elastic interaction of the crystals via the substrate.

* When the equilibrium shape of a 3D crystal (Volmer Weber case) is realised putting equation (d) in equation (e) of table II it follows the generalised Gibbs Thomson equation [87]:

$$
\Delta \mu=4 \gamma^{\prime} / \ell_{e q}+\mathrm{E}_{\mathrm{o}}\left[R\left(r_{e q}\right)+\left.r_{e q} \frac{\partial R}{\partial r}\right|_{r_{e q}}\right]
$$

Relation (66) says that an equilibrium crystal of finite size $\ell_{e q}$ has an excess chemical potential $\Delta \mu$ with respect to a bulk and non-strained crystal. The first term of (58) corresponds to a hyperbolical decrease of $\Delta \mu$ with size $\ell_{e q}$ as in the classical Gibbs-Thomson equation. The second term represents the contribution to chemical potential of the strained but relaxed crystal at its equilibrium shape ratio $\mathrm{r}_{\mathrm{eq}}$. For usual elasto-capillar length $\gamma / Y$ (see section 3.2.3.2.) this term contributes to less than $5 \%$ for misfits as high as $m_{0}=5.10^{-2}$. Thus the smaller the equilibrium shape $\ell_{e q}$, the higher the chemical overpotential. So at thermodynamic equilibrium, the usual Ostwald ripening still holds: small crystals loose molecules in favour of the bigger ones ${ }^{* * * * *}$. However kinetics when limited by surface diffusion may oppose to this thermodynamic tendency towards Ostwald ripening.

For coherent epitaxies a continuous layer is fully strained at its natural misfit $m_{o}$ (see section 3.2.3.1.). Thus a collection of islands initially relaxed, when coalescing, have to strain back to $m_{o}$ at layer completion. This was first mentioned by Cabrera [141] who said that this proceeds by the overlapping of the substrate strain fields when the island borders come closer. This means that, when close enough, two islands communicate by the substrate. This must affect the equilibrium shape of each crystal since the greater the coverage, the greater the

\footnotetext{
This may be different when the various crystals have different shapes. In this case, owing to its specific shape, a great crystal should have a greater chemical potential than a small one. In this case there could be a size selection.
} 
elastic energy. In [87] we have studied the equilibrium shape ratio of interacting box shaped crystals (Volmer Weber growth) and shown that interacting crystals have a shape ratio $r$ which deviates from that of isolated crystals. More precisely, the near equilibrium growing crystals prefer to thicken rather than to come closer to the borders and finally to coalesce. Obviously the harder the substrate, the smaller the deviation from the equilibrium shape of isolated crystals. Nevertheless these elastic interactions should also affect Ostwald ripening since now the mean elastic energy per atom that contributes to the total energy becomes coverage dependant. Floro et al. [142] tried to introduce such a contribution to the mean energy per atom by writing the usual chemical potential as $\Delta \mu=4 \gamma_{A}^{\prime}\left[1 / \ell_{e q}+p(\theta)\right]$. The mean field term $p(\theta)$ they introduced was obtained by finite elements calculations and found to be proportional to $\exp \left(\theta^{2}\right)-1$ where $\theta$ is the surface fraction covered by the islands. In this case the authors found that Ostwald ripening is enhanced by elastic repulsion as soon as elastic interaction in between deposited islands plays a role, that means for high coverage close to coalescence. The results thus obtained are compatible with their experiments [142].

- Such elastic interactions have also been proposed to be the driving force for selforganised growth $[143,144]$. Indeed, if in an array of islands one of the island deviates in size or shape, its neighbouring islands feel the change. It therefore installs a driving force for material transport restoring a uniform size and shape distribution, if temperature is high enough.

In the case of multilayers films, the repeated deposition of layers enhances the selforganisation, so 3D islands may organise progressively in a uniform and regular pattern [150]. The theoretical description of such organisation in multilayers has been given by Xie et al. [143] then Tersoff [145], and called vertically self-organised growth.

\section{ACKNOWLEDGEMENTS}

We thank A. Ranguis who has drawn some of the figures and helped us for editorial work.

\section{APPENDIX A}

In the case of a (001) biaxially strained $\left(\varepsilon_{1}=\varepsilon_{2}=\mathrm{m}_{0}\right)$ layer $\left(\sigma_{3}=0\right.$ at the free surface $)$ of a cubic material, the relation (3) can be written:

$$
\left(\begin{array}{c}
\sigma_{1} \\
\sigma_{2} \\
0 \\
0 \\
0 \\
0
\end{array}\right)=\left(\begin{array}{cccccc}
C_{11} & C_{12} & C_{12} & 0 & 0 & 0 \\
C_{12} & C_{11} & C_{12} & 0 & 0 & 0 \\
C_{12} & C_{12} & C_{11} & 0 & 0 & 0 \\
0 & 0 & 0 & C_{44} & 0 & 0 \\
0 & 0 & 0 & 0 & C_{44} & 0 \\
0 & 0 & 0 & 0 & 0 & C_{44}
\end{array}\right)\left(\begin{array}{c}
m_{o} \\
m_{o} \\
\varepsilon_{3} \\
0 \\
0 \\
0
\end{array}\right)
$$

where we use the $C_{i j}$ matrix for a cubic material $\left(\mathrm{x}_{1}, \mathrm{x}_{2}, \mathrm{x}_{3}\right.$ being the fourfold axis). The compliance matrix has the same form. For cubic material $S_{i j}$ and $C_{i j}$ are connected via the following relations [15]: 
$S_{11}=\frac{C_{11}+C_{12}}{\left(C_{11}+2 C_{12}\right)\left(C_{11}-C_{12}\right)} ; \quad S_{12}=\frac{-C_{12}}{\left(C_{11}+2 C_{12}\right)\left(C_{11}-C_{12}\right)} ; \quad S_{44}=1 / C_{44}$

Notice that $C_{\mathrm{ij}}$ and $\mathrm{S}_{\mathrm{ij}}$ coefficients can be inverted.

The previous relations between $\sigma$ and $\varepsilon$ thus give

$$
\left\{\begin{array}{l}
\sigma_{1}=\sigma_{2}=\left(C_{11}+C_{12}\right) m_{o}+C_{12} \varepsilon_{3} \\
2 C_{12} m_{o}+C_{11} \varepsilon_{3}=0
\end{array}\right.
$$

and thus

$$
\left\{\begin{array}{c}
\sigma_{1}=\sigma_{2}=\left(C_{11}+C_{12}-2 \frac{C_{12}^{2}}{C_{11}}\right) m_{o} \\
\varepsilon_{3}=-2 \frac{C_{12}}{C_{11}} m_{o}
\end{array}\right.
$$

The elastic energy density (5) thus reads

$$
w_{e l}=E_{c u b}^{2 D} m_{0}^{2}
$$

where $E_{(001)}^{2 D}=C_{11}+C_{12}-2 \frac{C_{12}^{2}}{C_{11}} \quad$ and $\quad v_{(001)}^{2 \mathrm{D}}=-2 \frac{C_{12}}{C_{11}}$ define the two dimensional Young's modulus and Poisson's ratio for this orientation $[19,20]$.

In the case of (111) strained layer one obtains $Y=6 C_{44}\left(C_{11}+2 C_{12}\right) /\left(C_{11}+2 C_{12}+4 C_{44}\right)$.

For less simple cubic orientations see [146].

\section{APPENDIX B}

The mathematical definition of the principal value (vp) is:

$v p\left[\int_{-\infty}^{\infty} \frac{f\left(x^{\prime}\right)}{x^{\prime}-x} d x^{\prime}\right]=\lim _{\delta \rightarrow 0}\left[\int_{-\infty}^{x-\delta} \frac{f\left(x^{\prime}\right)}{x^{\prime}-x} d x^{\prime}+\int_{x+\delta}^{\infty} \frac{f\left(x^{\prime}\right)}{x^{\prime}-x} d x^{\prime}\right]$ which can be written under the Hilbert form with $\mathrm{t}=\mathrm{x}-\mathrm{x}^{\prime} v p\left[\int_{-\infty}^{\infty} \frac{f\left(x^{\prime}\right)}{x^{\prime}-x} d x^{\prime}\right]=\lim _{\delta \rightarrow 0}\left[\int_{\delta}^{\infty} \frac{f(x+t)-f(x-t)}{t} d t\right]$. Thus in the case under study there is

$$
v p\left[\int_{-\infty}^{\infty} \frac{\sin \left(\omega x^{\prime}\right)}{x^{\prime}-x} d x^{\prime}\right]=\lim _{\delta \rightarrow 0}\left[\int_{\delta}^{\infty} \frac{\sin \omega(x+t)-\sin \omega(x-t)}{t} d t\right]=\lim _{\delta \rightarrow 0}\left[2 \cos \omega x \int_{\delta}^{\infty} \frac{\sin \omega t}{t} d t\right]
$$

Thus for $\omega>0$ there is $v p\left[\int_{-\infty}^{\infty} \frac{\sin \omega x^{\prime}}{x^{\prime}-x} d x^{\prime}\right]=\pi \cos \omega x$ 


\section{APPENDIX C}

By writing the strain tensor in terms of displacement:

$\frac{1}{2} \int_{V} \sigma_{i k} \varepsilon_{i k} d V=\frac{1}{2} \int_{V} \sigma_{i k} \frac{1}{2}\left[\frac{\partial u_{i}}{\partial x_{k}}+\frac{\partial u_{k}}{\partial x_{i}}\right] d V$

Integration by parts transforms the second member in a surface integral:

$\frac{1}{2} \int u_{i} \sigma_{i k} n_{k} d S-\frac{1}{2} \int \frac{\partial \sigma_{i k}}{\partial x_{k}} u_{i} d V$

The last integral is zero since in the bulk of the solid the bulk density of force components (1), $f_{i}=\partial \sigma_{i k} / \partial x_{k}$ have to be zero when no body forces as gravity or others are acting. In the first integral $n_{k}$ are the components of the unit vector normal to the surface so that $\sigma_{i k} n_{k}=f_{i}$ are surface force density components creating the displacement $w_{e l}=\frac{1}{2} \int_{S} f_{i} u_{i} d S$.

\section{APPENDIX D}

For semi-conductor films, the situation may appear simple since it seems that a film of roughly 1 to 3 monolayers can already be considered as an elastic continuum where bulk constants are roughly valid [147,148]. This may come from the short-range potential describing semi conductor bondings. For other materials the situation is more complex since now the bulk elastic properties must be size dependent. However a simple model of size dependence of the biaxial modulus of thin film has been published [71]. In this paper Streitz et al. have calculated the thickness-dependent biaxial modulus $\mathrm{Y}(\mathrm{h})$ of thin metal films (thickness $\mathrm{h}$ ) as the second derivative of the total energy $\Delta \mathrm{U}$ per unit volume with respect to strain. They show that for $\mathrm{Co}, \mathrm{Ni}, \mathrm{Ag}$ and Au with (001) or (111) orientations, size dependent biaxial moduli obtained from atomistic simulations are perfectly fitted by a simple analytical model where $\Delta \mathrm{U}=\Delta \mathrm{U}_{\mathrm{B}}+\Delta \mathrm{U}_{\mathrm{S}}$ with $\Delta \mathrm{U}_{\mathrm{B}}$ the volume strain density energy of an infinite material (characterised by the usual bulk biaxial modulus) and $\Delta \mathrm{U}_{\mathrm{S}}$ the work done against surface and interface stress. More precisely using their relation (18) the biaxial modulus of a thin supported film reads in the framework of the assumptions herein (linear elasticity, strain independent surface stress) $Y(h)=Y_{\infty}\left[1-\varepsilon_{/ /}(2 \eta-3)\right]$ with $\quad \varepsilon_{/ /}=-\frac{1-v_{A}}{E_{A}} \frac{s_{A}+s_{A B}}{h_{A}}$ and where $\eta=-\varepsilon_{z z} / \varepsilon_{x x}$ is a function of the Poisson ratio. Thus the biaxial modulus $\mathrm{Y}(\mathrm{h})$ of a thin film scales with the reciprocal of the film thickness and reaches the usual bulk value $\mathrm{Y}_{\infty}$ for increasing thickness. Thus in the framework of linear elasticity it seems that it is formally equivalent to use size-dependent bulk elastic constants or to properly consider surface stress. 


\section{APPENDIX E}

The proof is: the short range behaviour reads $\Phi(z)=\Phi_{\infty} \prod_{0}^{z}(z-1), \prod$ the Heaviside function and from (32) with $\theta=0, \mathrm{~V}=0$

$$
\frac{\partial \Delta F}{\partial z}=-\left(\Delta \mu-\mathrm{E}_{0}\right) L^{2} a+L^{2} \frac{\partial \Phi(z)}{\partial z}=0
$$

so that now in table II for $\Phi_{\infty}<0$ there is $\delta(z-1)=\frac{\Delta \mu-\mathrm{E}_{o}}{-\left|\Phi_{\infty}\right|}$. Since $\Delta \mu<\mathrm{E}_{o}$ the ratio is positive so that the solution is $\mathrm{z}^{*}=1$ and also for $\Delta \mu=0, \mathrm{z}_{0}=1$.

\section{APPENDIX F}

From a thermodynamical point of view the number of interfacial dislocations may pass from $\mathrm{N}$ to $\mathrm{N}+1$ when the total energy change due to the introduction of the $(\mathrm{N}+1)^{\text {th }}$ dislocation is negative. The elastic energy stored by the system of $2 \mathrm{xN}$ orthogonal dislocations exist can be roughly written [80] for isotropic solids

$$
E_{N}=2 \frac{m_{o}-m^{\prime}}{a} b^{2} \frac{E_{A B}}{2 \pi}(1+\ln \lambda) S_{A B}+Y m^{\prime 2} V R
$$

where the first term is the energy of a double array of perpendicular non interacting dislocations with $1 / \mathrm{E}_{\mathrm{AB}}=1 / \mathrm{E}_{\mathrm{A}}+1 / \mathrm{E}_{\mathrm{B}}$ the reciprocal "interfacial modulus" $\left(\mathrm{E}_{\mathrm{A}}=\mathrm{Y}_{\mathrm{A}} /\left(1-\mathrm{v}_{\mathrm{A}}\right)\right.$ and $E_{B}=Y_{B} /\left(1-v_{B}\right)$ are the elastic modulus of $A$ and $B$ respectively), $b$ the Burgers vector component in the interface, $\mathrm{m}_{0}-\mathrm{m}$ ' the part of the misfit accommodated by the $(\mathrm{N}+1)^{\text {th }}$ dislocation pair and $\lambda$ a cut off. When $\mathrm{h}<\mathrm{d}$ where $\mathrm{d}$ is the equidistance in between dislocations there is $\lambda=h$, if not there is $\lambda=\mathrm{d} / 2$. When there are $\mathrm{N}$ interfacial dislocations the released elastic misfit is obtained from Vernier considerations. It reads

$$
m^{\prime}=m_{0}-N b / \sqrt{\left.S_{A B}\right|_{N}}
$$

where $\left.S_{A B}\right|_{N}$ is the interfacial area for a crystal having $\mathrm{N}$ interfacial dislocations. The thermodynamical criterion for the $(\mathrm{N}+1)^{\text {th }}$ dislocation entrance is thus obtained from $E_{N+1}-E_{N}<0$ that means when

$$
\frac{h}{a}=\frac{V}{\left.S_{A B}\right|_{N}}>\frac{b}{a} \frac{1}{2 \pi} \frac{K}{1+K} \frac{1+\ln \lambda}{\left.m_{o}-\frac{N+1 / 2}{\sqrt{\left.S_{A B}\right|_{N}} / b}\right) R}
$$

Where $\mathrm{K}=\mathrm{E}_{\mathrm{B}} / \mathrm{E}_{\mathrm{A}}$ is the relative rigidity, and $\mathrm{R}$ the relaxation factor. For a thin pseudomorphous infinite film there is $\mathrm{R}=1, S_{A B} \rightarrow \infty$ and $\lambda=\mathrm{h} / \mathrm{a}$ so that the previous relation reads

$$
\frac{h}{a}>\frac{b}{a} \frac{1}{2 \pi} \frac{K}{1+K} \frac{1+\ln (h / a)}{m_{o}}
$$

which is nothing other than the usual Matthews relation giving the critical height beyond which dislocations may appear in a pseudomorphous film [80]. Let us note that more precise expressions of $\mathrm{h}$ have been given in literature (for a review see [149]) but above relations roughly give the good order for reasonable misfits (a few \%). 


\section{APPENDIX G}

The elastic energy of a film in presence of surface stress can be written

$$
E_{\text {film }}\left(m^{\prime}\right)=\mathrm{E}_{0} m^{\prime 2} h_{A}+2 m^{\prime} \Delta s^{\infty}\left(1-\exp \left(-h_{A} / a \zeta\right)\right.
$$

The first term is the bulk elastic energy stored by the film of height $h_{A}$. The second term is the work against surface and interface stresses corrected for long range exponential inter layers forces $[69,70]$. In this latter term $\Delta s^{\infty}=s_{A}^{\infty}+s_{A B}^{\infty}-s_{B}^{\infty}$ is the surface stress change due to the film. The value of $m^{\prime}$ which minimises $\mathrm{E}_{\text {film }}+\mathrm{E}_{\mathrm{disl}}$ is: $\quad m^{\prime}=m_{o}^{\prime}-\frac{\Delta s^{\infty}\left(1-\exp \left(-h_{A} / \zeta a\right)\right)}{\mathrm{E}_{\mathrm{o}}} \frac{1}{h_{A}}$ With $m_{o}^{\prime}=\frac{1}{h_{A}}\left[\frac{b}{2 \pi} \frac{K}{1+K}\left(1+\ln \left(\frac{h_{A}}{b}\right)\right)\right]$ where $K=E_{B} / E_{A}$ is the relative rigidity of the substrate with respect to deposit and where we put for the cut off distance $\lambda=\mathrm{h}_{\mathrm{A}}$ in the expression of $\mathrm{E}_{\mathrm{dis}}$.

The film thickness $h_{c}$ beyond which the first dislocation entrance becomes energetically favourable is then obtained by setting $\mathrm{m}^{\prime}=\mathrm{m}_{\mathrm{o}}$ in the previous relation. Thus there is:

$$
h=\frac{1}{\left|m_{o}\right|} \frac{b}{2 \pi} \frac{K}{1+K}\left[1+\ln \left(\frac{h}{b}\right)\right]-\frac{1}{m_{o}} \frac{\Delta s^{\infty}(1-\exp (-h / \zeta a))}{\mathrm{E}_{0}}
$$

The critical thickness is therefore amended (compare with last equation in appendix $F$ ) by the surface stress effect.

\section{APPENDIX H}

As known [6,92] homogeneous classical nucleation barrier amounts to $1 / 3$ of the total surface energy of the nucleus :

$$
\frac{\Delta F^{*}}{k T}=\frac{1}{3} \gamma_{A} \frac{6(\ell / a)^{2}}{k T}=\frac{2 \gamma_{A} a^{2}}{k T}(N *)^{2 / 3}
$$

with $\mathrm{N}^{*}$ the number of molecules in the critical nucleus.

From $\frac{\partial \Delta F}{\partial N^{*}}=0$ there is

$$
N^{*}=\left(\frac{4 \gamma_{A}}{\Delta \mu}\right)^{3} \text { and } \frac{\Delta F^{*}}{k T}=\frac{a^{2}}{2 k T} \frac{\gamma_{A}^{3}}{\Delta \mu^{2}}
$$

The nucleation rate reads $d N * / d t \approx v \exp [-\Delta F * / k T]$ with $v=10^{13} \mathrm{sec}^{-1}$ an attempt frequency. Thus a nucleation rate $d N * / d t \approx 1$ which is quite reasonable gives

$$
\Delta F * / k T \approx 30
$$

Since $2<\left(2 \gamma_{A} a^{2}\right) / k T<5$ is a quite usual surface energy at evaporating temperature the operative critical nucleus contains

$$
15<\mathrm{N}^{*}<60
$$

molecules. From (ii) it results the supersaturation range $3 k T / a^{2}<\Delta \mu<5 k T / a^{2}$ or compared to $\mathrm{E}_{o}, \Delta \mu / \mathrm{E}_{o}=\Delta \mu /\left(\operatorname{Yam}_{o}^{2}\right), \mathrm{E}_{o}=10^{12} \mathrm{erg} \mathrm{cm}^{-3}, \mathrm{~m}_{\mathrm{o}}=2.10^{-2}$ gives at $\mathrm{T} \approx 10^{3} \mathrm{~K}$

$$
6<\frac{\Delta \mu}{\mathrm{E}_{o}}<10
$$


Non-classical nucleation is that one where the nucleus size $\mathrm{N}^{*}$ is so small $\left(\mathrm{N}^{*}=1,2,3 \ldots\right)$ that the macroscopic concepts of surface energy no more holds. Then additive bond energies are convenient to define each cluster (see Walton's simple theory in $[92,106]$ or papers as [113].

\section{APPENDIX I}

Exact calculations of the dipole moment $\mathrm{A}_{\mathrm{ij}}$ of adsorbed atoms on a substrate can be done precisely when the interactions in-between two ions (i) and (j), $x^{i, j}$ apart, are well represented by a pair potential $\Phi\left(x^{i, j}\right)$. Indeed, using the concept of point forces the components of the force of an atom (i) acting at $x^{i, j}$ is : $f_{\alpha}^{i, j} \delta\left(\vec{x}-\vec{x}^{i, j}\right)$ with $f_{\alpha}^{i, j}=-\frac{\partial \Phi\left(x^{i, j}\right)}{\partial x^{i, j}} \frac{x_{\alpha}^{i, j}}{\left|\vec{x}^{i, j}\right|}$. Far from the point of application of the force $x>x^{i, j}$ this force reads up to the first order $f_{\alpha}^{i, j} \delta\left(\vec{x}-\vec{x}^{i, j}\right)=f_{\alpha}^{i, j}\left[\delta(\vec{x})+\sum_{\beta} x_{\beta}^{i, j} \frac{\partial}{\partial x_{\beta}^{i, j}} \delta(\vec{x})\right]$. For all i-j bonds the total distribution of forces thus reads $F_{\alpha}^{i}\left(\vec{x}-\vec{x}^{i}\right)=\sum_{j} f_{\alpha}^{i, j}\left[\delta(\vec{x})+\sum_{\beta} x_{\beta}^{i, j} \frac{\partial}{\partial x_{\beta}^{i, j}} \delta(\vec{x})\right]$. At mechanical equilibrium the first term of the development vanishes and there is $F_{\alpha}^{i}\left(\vec{x}-\vec{x}^{i}\right)=A_{\alpha \beta} \frac{\partial}{\partial x_{\beta}^{i, j}} \delta(\vec{x})$ with the moment of the elastic dipole $A_{\alpha \beta}=-\sum_{j} \frac{\partial \Phi\left(x^{i, j}\right)}{\partial x^{i, j}} \frac{x_{\alpha}^{i, j} x_{\beta}^{i, j}}{\left|\vec{x}^{i, j}\right|}$

In [26] appendix IV one can find for an ion self adsorbed, but non relaxed on the (001) face of the NaCl-structure type : $\mathrm{A}_{\mathrm{ii}}=0.10 \mathrm{e}^{2} / \mathrm{a} \pm 2 \%, \mathrm{i}=1,2$ with a the shortest equilibrium distance between opposite ions, $\mathrm{e}^{2}=1.510^{-7} \mathrm{eVcm}$ per ion so that for $\mathrm{a}=210^{-8} \mathrm{~cm} \mathrm{~A} \mathrm{~A}_{\mathrm{ii}}=0.75 \mathrm{eV}(\mathrm{i}=1,2)$. More generally, comparing with the cohesion energy of this structure type $W_{\text {coh. }}=\frac{1}{2} M \frac{e^{2}}{a}\left(1-\frac{1}{m}\right)$ with $\mathrm{M}=1.7486 \pm 0.05 \%$ the Madelung number per ion, $\mathrm{m}$ the Born repulsion exponent $(8<\mathrm{m}<12)$. Therefore $A_{i i} / W_{c o h}=\frac{0.20}{M} \frac{m}{m-1}, \mathrm{i}=1,2$ or the narrow estimation valid for all the alkali-halide series, $0.12<A_{i i} / W_{c o h}<0.13(\mathrm{i}=1,2)$. Notice the peculiarity of the (001) faces $A_{33} / W_{\text {coh. }}<10^{-3}$.

More crude estimations have been done with Lennard-Jones (6-12) interactions. [25] gives $\mathrm{A}=4.5 \mathrm{eV}$ for $\mathrm{Xe}$ on (111)Au, [23] gives $\mathrm{A}=0.23 \mathrm{eV}$ for $\mathrm{Ar} /(111) \mathrm{Ar}$. Finally Duport et al. (see [124] appendix A2) scaled $A_{11} / W_{\text {coh. }}=0.17$ and $A_{22} / W_{\text {coh. }}=0.07$ for the self adsorbed atom on the border of a hypothetic compact two-dimensional crystal. 


\section{APPENDIX J}

Using (12), the expression (17) of the elastic interaction of 2 elastic defects (1) and (2) (located at the surface $\mathrm{z}=0$ ) reads:

$$
U=\frac{1}{2} \sum_{\alpha} \sum_{\beta} \iint F_{\alpha}^{(1)}(\vec{x}) D_{\alpha \beta}\left(\vec{x}, \vec{x}^{\prime}\right) F_{\beta}^{(2)}\left(\vec{x}^{\prime}\right) d V d V^{\prime}
$$

which when forces have only x components reads

$$
U=\frac{1}{2} \sum_{x} \sum_{x} \iint F_{x}^{(1)}(\vec{x}) D_{x x}\left(\vec{x}, \vec{x}^{\prime}\right) F_{x}^{(2)}\left(\vec{x}^{\prime}\right) d V d V
$$

with $D_{x x}\left(\vec{x}, \vec{x}^{\prime}\right)=D_{x x}\left(x, x^{\prime}\right)=\frac{1-v^{2}}{\pi E}\left[\frac{1}{r}+\frac{v}{1-v} \frac{\left(x-x^{\prime}\right)^{2}}{r^{3}}\right][13]$

and $r=\left[\left(x-x^{\prime}\right)^{2}+\left(y-y^{\prime}\right)^{2}+(2 a)^{2}\right]^{1 / 2}$ the in-plane distance in between the two defects. A cut off distance $2 \mathrm{a}$ is introduced to avoid local divergences.

For our purpose the step on a stressed body is described by an elastic monopole whose $\mathrm{x}$ component (perpendicular to the step and directed towards the lowest terrace) reads $F_{x}^{m o}(\vec{x})=F \delta(\vec{x})$ with $F==\frac{E a}{1-v^{2}} m_{o} a$ whereas the elastic dipole is described by $F_{x}^{d i}(\vec{x})=A \frac{\partial}{\partial x} \delta(\vec{x})$ where $\delta(\vec{x})$ is the Dirac function. Thus injecting these expressions in (i) one obtains easily by using substitution properties of the Dirac function:

$$
U^{m o-m o}=\frac{1}{2} F^{2} D_{x x}\left(x^{(1)}, x^{(2)}\right) ; U^{m o-d i}=\left.\frac{1}{2} F A \frac{\partial}{\partial x^{(1)}} D_{x x}\left(x, x^{\prime}\right)\right|_{x^{(1)}, x^{(2)}} ; U^{d i-d i}=\left.\frac{1}{2} A^{2} \frac{\partial^{2}}{\partial x^{(1)} \partial x^{(2)}} D_{x x}\left(x, x^{\prime}\right)\right|_{x^{(1)}, x^{(2)}}
$$

The interaction in between a lines of defects and a single defect located at a distance $\mathrm{x}=\mathrm{L}$ of the line is thus obtained by integrating (ii) along the line $y$ with $\mathrm{x}=\mathrm{L}$. Then the interaction in between two lines of defects is obtained by multiplying the previous result by the number of defects in the second line. The expressions of table III are the first order development of these expressions for $\mathrm{y} \rightarrow \infty$.

\section{REFERENCES}

1. L. Royer, Bull. Soc. Fr. Min. Crist., 51 (1928) 7.

2. S. Jain and W. Hayes, Semic. Sci. Techn., 6, (1991) 547.

3. D.Sander, R. Skomski, A.Enders, C.Schmidthals, D. Reuter and J.Kirschner, J. Phys. D, Appl. Phys. 31 (1998) 663.

4. G.Wulf, Z.Krist. 34 (1901) 449.

5. C. Herring in Gomer and Smith Eds. Structure and Properties of Solid Surface Univ. Chicago, Press 1953.

6. J.W. Gibbs in The collected works of J.W. Gibbs, p.314 (Longmans, Green and co, New York, 1928).

7. R. Shuttleworth, Proc. Roy. Soc. London 163, 644 (1950).

8. A.F. Andreev, Y.A. Kosevitch, JETP 54, (1981) 761.

9. P. Nozières, D.E. Wolf, Z. Phys B 70 (1988) 399, (1988) 507.

10. P. Nozières, D.E. Wolf, Z. Phys B 70 (1988) 507. 
11. E.Bauer, Z.Krist 110 (1958) 372.

12. E.Bauer, Z.Krist 110 (1958) 395.

13. L.D. Landau and E.M. Lifshitz, Theory of elasticity (Oxford Pergamon) 1970.

14. A. Love "A treatise on the mathematical theory of elasticity" (New York, Dover publication 1927).

15. J.F. Nye, Physical properties of crystals (Oxford, University press) 1985.

16. A. Angot, "Compléments de mathématiques" (Ed. revue d'optique, Paris) $4^{\circ}$ edition, 1961 page $272-282$.

17. H.B. Huntington, "the elastic constants of crystals" in Solid state Physics vol. 7 (Academic Press, New York) 1958, 213.

18. R.F. Hearmon, Rev. of Modern physics, 18 (1946) 409.

19. D. Sanders, Rep. Prog. Phys. 62 (1999) 809.

20. W.A. Brandtley, J. Appl. Phys. 44, (1973) 534.

21. R. Kern, in I. Sunagawa (Ed.) The equilibrium form of a crystal in Morphology of crystals Vol A, Terra Tokyo (1987) 79.

22. R.D. Mindlin, Physics, 7, (1936) 195.

23. A. Maradudin, R. Wallis, Surf. Sci. 91 (1980) 423.

24. V.I.Marchenko, A.Y.Parshin, JETP 52 (1980) 129.

25. K.Lau, W.Kohn, Surf. Sci. 65 (1977) 607.

26. R.Kern, M.Krohn, Phys. Stat. Sol. 116 (1989) 23.

27. P. Nozières, Lectures at the Beg Rohu Summer School, Solid far from equilibrium (Cambridge University Press, Ed. C. Godrèche) 1993.

28. J.M. Rickman, D.J. Srolovitz, Surf. Sci. 284 (1993) 211.

29. W. Kappus, Z. Phys B 29 (1978) 239.

30. W. Kappus, Z. Phys B 38 (1980) 263.

31. P. Peyla, A. Vallat, C. Misbah, J. Cryst. Growth 201/202 (1999) 97

32. P. Peyla, A. Vallat, C. Misbah, H. Müller-Krumbhaar, Phys. Rev. Lett. 82 (1999) 787.

33. R.J. Asaro, W.A. Tiller, Metall. Trans. 3 (1972) 1789.

34. M.A. Grienfeld, Dokl. Akad. Nauk. SSSR 283 (1985) 1139.

35. M.A. Grienfeld, J. of Intelligent Material Systems and Structures, 4 (1993) 76.

36. H. Gao, J. Mech. Phys. Solids, 39 (1991) 443.

37. W.H. Yang, D.J. Srolovitz, J. Mech. Phys. Solids, 42 (1994) 1551.

38. L.B. Freund, Int. J. Solids Structures 32 (1995) 911.

39. D.J. Srolovitz Acta Metall. 37 (1989) 621.

40. B.J. Spencer, P.W. Voorhees, S.H. Davis, J. App. Phys. 73 (1993) 4955.

41. B.J. Spencer, D.I. Meiron, Acta Metall. 42 (1994) 3629.

42. M. Thiel, A. Willibald, P. Evers, A. Levchenlo, P. Leiderer, S. Balibar, Europhys Lett. 20 (1992) 707.

43. R.H.Torii, S. Balibar, J. of Low Temperature Physics, 1/2 (1992) 391.

44. V.Marchenko, JETP 54 (1981) 605.

45. F.C. Frank, A.N. Stroh, Proc. Phys. Soc. B65 (1952) 811.

46. J. Grilhe, Acta Metall.Mater., 37 (1993) 909.

47. C.H. Wu, J.Hsu, C.H.Chen, Acta. Mater 11 (1998) 3755.

48. K. Kassner, C. Misbah, Europhys. Lett. 28 (1994) 245.

49. O. Alerhand, D. Vanderbilt, R. Meade, J. Joannopoulos, Phys. Rev. Lett. 61 (1988) 1973.

50. F. Hen, W. Pachard, M. Webb, Phys. Rev. Lett, 61 (1988) 21

51. B. Croset, C. de Beauvais, Phys. Rev. B, 61 (2000) 3039 
52. E. Williams, R. Phaneuf, N. Bartelt, Mat. Res. Soc. Symp. Proc. 238 (1992) 219

53. E. Williams, Surf. Sci. 299/300 (1994) 502

54. E. Bauer, Appl. Surf. Sci. 11/12 (1982) 479.

55. M. Dupré, Théorie mécanique de la chaleur, Paris, (1969)

56. P. Müller, R. Kern, Appl. Surf. Sci. 102 (1996) 6

57. I. Stranski, L. Krastanov, Sitz. Ber. Akad. Wiss Wien (1938) 145

58. R. Kern, P. Müller, J.Cryst. Growth 145 (1995) 193.

59. J. Tersoff, R. Tromp, Phys. Rev. Lett. 70 (1993) 2782.

60. C. Duport, C. Priester, J. Villain in Morphological organisation in epitaxial growth and removal. Ed. Z. Zhang, M. Lagally, World Scientific (1998).

61. S. Christiansen, M. Albrecht, H. Strunk, P. Hansson, E. Bauser Appl. Phys. Lett 66 (1995) 574.

62. E. Pehlke, N. Moll, A. Kley, M. Scheffler Appl. Phys.A 65 (1997) 525.

63. L. Freund, H. Johnson, R. Kukta, MRS bull. 399 (1996) 259.

64. D. Wong, M. Thouless J. Mater. Sci. 32 (1997) 1835.

65. P. Müller, R. Kern, Surf. Sci. 457 (2000) 229.

66. R. Kern, JJ. Métois, G. Lelay in Current Topic in Material Science vol. 3, Kaldis (Ed.) North Holland, Amsterdam, 1979, p. 196.

67. D. Nenow and A. Trayanov, J. Cryst. Growth 79, (1986) 801.

68. J.G. Dash, Cont. Phys. 30, (1989) 801.

69. P. Müller, R. Kern, Appl. Surf. Sci. 102 (1996) 6.

70. P. Müller, O. Thomas, Surf. Sci. Lett., 465 (2000) L 764.

71. F.H. Streitz, R.C. Cammarata and K. Sieradzki, Phys. Rev. B 49, 10699 (1994).

72. C. Duport, "Elasticité et croissance cristalline" PhD University J. Fourier, Grenoble 1996.

73. S.M. Hu, J. Appl. Phys. 50, 4661 (1979).

74. R. Kern, P. Müller, Surf. Sci. 392 (1997) 103.

75. R. Kern, JJ. Métois, G. Lelay in Current Topic in Material Science vol. 3, Kaldis (Ed.) North Holland, Amsterdam, 1979, p. 131-419.

76. A. Thomy, X. Duval, Surf. Sci. 299/300415 (1994) 797.

77. J.W. Schultze, D. Dickerman, Surf. Sci. 54 (1976) 489.

78. G. Gerth, V. Abelman, Cryst. Res. Techn. 24 (1989) 35.

79. P. Müller, R. Kern, A. Ranguis, G. Zerwetz, Eur. Phys. Lett. 26 (1994) 461.

80. J.W. Matthews in Dislocations in Solids, F. Nabarro (Ed.), Vol. 2 (1989) 461. and in Epitaxial Growth vol. A and B, Materials Science Series, (Acad. Press) (1975).

81. J. Tersoff, F.K. LeGoues, Phys. Rev. Lett. 72, 3570 (1994).

82. R. Kaishew, Bull. Acad. Sc. Bulg. (Ser. Phys.) 2 (1951) 191.

83. R. Kaishew, Arbeitstatung Festköper Physik, Dresden (1952) 81.

84. R. Kaishew, Comm. Bulg. Acad. Sci. 1 (1950) 10.

85. R. Kaishew, Fortscht. Miner. 38 (1960).

86. W. Winterbottom, Acat. Metall. 15 (1968) 303.

87. P. Müller, R. Kern, J. Cryst. Growth 193, (1998) 257.

88. P. Müller, R. Kern, Appl. Surf. Sci. 162/163 (2000) 133.

89. P. Müller, R. Kern, Microsc. Microanal. Microstruct. 8 (1997) 229.

90. M. Hammar, F. Legoues, J. Tersoff, M.C. Reuter, R. Tromp, Surf. Sci. 349 (1996) 129.

91. F. Legoues, M. Hammar, M. Reuter, R. Tromp, Surf. Sci. 349 (1996) 249.

92. I.V. Markov, Crystal growth for beginners, World Scientific, Singapore (1995).

93. P. Müller, R. Kern, Appl. Surf. Sci. 164 (2000) 68. 
94. J. Floro, E. Chason, R. Twesten, R. Hwang, L. Freund, Phys. Rev. Lett. 79 (1997) 3946.

95. J. W.Cahn, Acta Metall. 28 (1980) 1333.

96. N. Moll, M. Scheffler, E. Pehlke, Phys.Rev. B 58 (1998) 4566.

97. Y. Mo, D. Savage, B. Schwartzentruber, M. Lagally, Phys. Rev. Lett. 65 (1990) 1020.

98. C. Aumann, Y. Mo, M. Lagally, Appl. Phys. Lett. 59 (1991) 1061.

99. D. Steigerwald, I. Jacob,W. Egelhoff, Surf. Sci. 202 (1988) 472.

100. M. Copel, M.C. Reuter, E. Kaxiras, R. Tromp, Phys. Rev. Lett. 63 (1989) 632.

101. D. Eaglesham, F. Unterwald, D. Jacobson, Phys. Rev. Lett 70 (1993) 966.

102. H. Ibach, Surf. Sci. Reports, 29 (1997) 193.

103. H. Ibach, Surf. Sci. Reports, 35 (1999) 71.

104. D. Sander and H. Ibach in Landolt-Börnstein New Series vol. III/42, Physics of covered solid surfaces, H. Bonzel (Ed.).

105. W.K. Burton, N. Cabrera, F.C. Frank Phil. Trans. Roy. Soc. 243 (1951) 299.

106. J. Villain, A.Pimpinelli, "Physique de la croissance cristalline" Aléa Saclay Eyrolles (Ed. C. Godréche) (1995).

107. S. Stoyanov, D. Kashiev, in "Current topics in material Science" vol. 7 Ed. E. Kaldis (North Holland Publishing) 1981, 69.

108. J.J. Harris, B.A. Joyce, Surf. Sci. 103 (1981) L90.

109. Y. Horio, A. Ichimaya, Surf. Sci. 298 (1993) 261.

110. A.R. Miedena, Z. Metallkde, 69 (1978) 287.

111. G. Zinsmeister, Thin Solid Films, 2 (1968) 497.

112. G. Zinsmeister, Thin Solid Films, 7 (1971) 51.

113. J. Venables, Phil. Mag. 27 (1973) 697.

114. F. Binsberger, in Crystal growth, ICGG III Conference, North Holland Pub. (1971) 44.

115. G. Gilmer, P. Bennema, ICGG III Conference, North Holland Pub. (1971) 147.

116. H.J. Leamy, G.H. Gilmer, J. Cryst. Growth 24/25 (1974) 499.

117. C. Ratsch, P. Smilauer, D. Vvedensky, A. Zangwill, J. de Phys. I, 6 (1996) 575.

118. C. Ratsch, A. Zangwill, P. Smilauer, Surf. Sci 314 (1994) L942.

119. C. Ratsch, A. Zangwill, Appl. Phys. Lett. 63 (1993) 2348.

120. R.L. Schwoebel, E.J. Shipsey, J. Appl. Phys. 37 (1966) 3682.

121. G. Ehrlich, Surf. Sci. 63 (1977) 422.

122. P. Bennema and G.H. Gilmer in Crystal Growth Ed. P. Hartman, North Holland, 1973, p. 263-327.

123. C. Duport, P. Nozières, J. Villain, Phys. Rev. Lett. 74 (1995) 134.

124. C. Duport, P. Paoliti, J. Villain, J. de Phys. I, 5 (1995) 1317.

125. J. Tersoff, Phys. Rev. Lett. 75 (1995) 2730.

126. E. Gruber et M. Mullins, J. Phys. Chem. Solids 28 (1967) 875.

127. J.J. Métois, S. Stoyanov, Surf. Sci. 440 (1999) 407.

128. M. Schroeder, D. Wolf, Surf. Sci. 375 (1997) 129.

129. C. Lei, C. Jia, J. Lisoni, M. Siegert, J. Schubert, C. Buchal, K. Urban, J. Cryst. Growth, in press(2000).

130. F. Glas, Phys. Rev. B, 55 (1997) 11277.

131. F. Glas, Appl. Surf. Sci. 123/124 (1998) 298.

132. F. Ross, J. Tersoff, R. Tromp, Phys. Rev. Lett. 80 (1998) 984.

133. F. Glas, J. Appl . Phys. 62 (1987) 3201.

134. F. Glas, J. Appl. Phys. 70 (1991) 3556.

135. I. Patova, V. Malyshkin, V. Schukin, J.Appl. Phys. 74 (1993) 7198.

136. J.E. Guyer, P.M. Voorhes, Phys. Rev. Lett. 74 (1995) 4031. 
137. B.J. Spencer, D.I. Meiron, Acta Met. Mater 42 n $^{\circ} 11$ (1994) 3629.

138. J.E. Guyer, P.M. Voorhes, J. Cryst. Growth 187 (1998) 149.

139. C. Priester, J. Vac. Sci. Technol. B 16 (1998) 2421.

140. J. Tersoff, Phys. Rev. Lett. 81 (1998) 3183.

141. N. Cabrera, Mem. Sci. Rev. Metal. Fr., 62 (1965) 205.

142. J. Floro, M. Sinclair, E. Chason, L. Freund, R. Twesten, R. Hwang, G. Lucadamo, Phys. Rev. Lett. 84 (2000) 701.

143. Q. Xie, A. Madhukar, P. Chen, N. Kobayashi, Phys. Rev. Lett. 75 (1995) 2542.

144. V.A. Shukin, N.N. Ledentsov, P.S. Kop'ev, D. Dimberg, Phys. Rev. Lett., 75 (1995) 2968.

145. J. Tersoff, C. Teichert, M. Lagally, Phys. Rev. Lett. 76 (1996) 1675.

146. L. De Caro, L. Tapfer, Phys. Rev. B 48 (1993) 298.

147. O. Brandt, K. Ploog, R. Bierwolf, M. Hokenstein, Phys. Rev. Lett. 68 (1992) 1339.

148. J.C. Woicik, J.G. Pellegrino, S.H. Southworth, P.S. Shaw, B.A. Karlin, C.E. Bourlin, K.E. Miyano, Phys. Rev. B 52 (1995) 52.

149. D.J. Duntan, J. of Mat. Sci. : Mat. in Electronics, 8 (1997) 33.

150. J.Moison, F.Houzay, F.Barthe, L.Leprince, E.André, O.Vatel, Appl. Phys. Lett. 64 (1994) 19. 